\title{
Physical Processes in Star Formation
}

\author{
Philipp Girichidis • Stella S. R. Offner • \\ Alexei G. Kritsuk • Ralf S. \\ Klessen • Patrick Hennebelle • \\ J. M. Diederik Kruijssen • Martin G. H. \\ Krause • Simon C. O. Glover • Marco \\ Padovani
}

Received: date / Accepted: date

\begin{abstract}
Star formation is a complex multi-scale phenomenon that is of significant importance for astrophysics in general. Stars and star formation are key pillars in observational astronomy from local star forming regions in the Milky Way up to high-redshift galaxies. From a theoretical perspective, star formation and feedback processes (radiation, winds, and supernovae) play a pivotal role in advancing our understanding of the physical processes at work, both individually and of their interactions. In this review we will give an
\end{abstract}

Philipp Girichidis

Leibniz-Institut für Astrophysik (AIP) - Potsdam, Germany E-mail: philipp@girichidis.com

Stella S. R. Offner

The University of Texas at Austin - Austin TX, USA

Alexei G. Kritsuk

University of California, San Diego; La Jolla, California, 92093-0424, USA

Ralf S. Klessen

Universität Heidelberg, Zentrum für Astronomie, Institut für Theoretische Astrophysik, Albert-Ueberle-Str. 2, 69120 Heidelberg, Germany

Patrick Hennebelle

Laboratoire AIM, Paris-Saclay, CEA/IRFU/SAp CNRS Université Paris Diderot,91191 Gif-sur-Yvette Cedex, France LERMA (UMR CNRS 8112), Ecole NormaleSupérieure, 75231 Paris Cedex, France

J. M. Diederik Kruijssen

Astronomisches Rechen-Institut, Zentrum für Astronomie der Universität Heidelberg - Heidelberg, Germany

Martin G. H. Krause

Centre for Astrophysics Research, School of Physics, Astronomy and Mathematics, University of Hertfordshire, College Lane, Hatfield, Hertfordshire AL10 9AB, UK

Simon C. O. Glover

Universität Heidelberg, Zentrum für Astronomie, Institut für Theoretische Astrophysik, Albert-Ueberle-Str. 2, 69120 Heidelberg, Germany

Marco Padovani

INAF-Osservatorio Astrofisico di Arcetri - Largo E. Fermi, 5 - 50125 Firenze, Italy 
overview of the main processes that are important for the understanding of star formation. We start with an observationally motivated view on star formation from a global perspective and outline the general paradigm of the life-cycle of molecular clouds, in which star formation is the key process to close the cycle. After that we focus on the thermal and chemical aspects in star forming regions, discuss turbulence and magnetic fields as well as gravitational forces. Finally, we review the most important stellar feedback mechanisms.

Keywords star formation - basic processes - global star formation laws · stellar feedback

\section{Introduction}

In this section we provide a general overview of the star formation process on global scales as well as the current paradigm of the life cycle of molecular clouds, in which the formation of stars marks the turning point between the cooling of gas, the condensation and finally the collapse on the one hand and the feedback processes on the other hand, in which the stars influence their environment and reheat the gas to complete the cycle. There are large variety of physical processes involved star formation, which interact in a complicated and highly non-linear manner and on very different spatial and dynamical scales. Nonetheless, the global process of star formation in galaxies seems to follow rather simple relations, which points towards a self-regulated rather than an unstable or chaotic process.

\subsection{Star formation in galaxies}

Star formation takes place in molecular clouds (Kennicutt and Evans, 2012). As a result, galaxies exhibit a tight relation between the star formation rate (SFR) surface density, $\Sigma_{\mathrm{SFR}}=\equiv \int_{-\infty}^{\infty} \rho_{\mathrm{SFR}}(z) \mathrm{d} z$ (typically expressed in units of $\mathrm{M}_{\odot} \mathrm{yr}^{-1} \mathrm{kpc}^{-2}$ ), and the (molecular) gas surface density, $\Sigma \equiv \int_{-\infty}^{\infty} \rho_{\text {gas }}(z) \mathrm{d} z$ (typically expressed in units of $\mathrm{M}_{\odot} \mathrm{pc}^{-2}$ ) (Schmidt, 1959; Kennicutt, 1989, 1998), where $z$ is the coordinate perpendicular to the galactic disc. In a spatially-resolved sense, this 'star formation' relation persists down to scales of $\sim 500$ pc (Bigiel et al., 2008; Kennicutt et al., 2007; Leroy et al., 2013), below which the stochasticity introduced by the time evolution of individual molecular clouds and star-forming regions causes the relation to break down (Schruba et al., 2010; Liu et al., 2011; Feldmann et al., 2011; Kruijssen and Longmore, 2014). The star formation relation follows a power law $\Sigma_{\mathrm{SFR}} \propto \Sigma^{N}$ with $N=1.0-1.5$ and a normalisation implying a molecular 'gas depletion time' (i.e. the time required to turn the entire gas reservoir into stars at the current SFR) of $t_{\text {dep }} \equiv \Sigma / \Sigma_{\mathrm{SFR}} \sim 2$ Gyr. Efforts to physically interpret this relation have focused on two main questions. 
1. Which physical processes set the slope of the star formation relation? A power close to $N=1.5$ suggests that the free-fall time (related to the gas volume density as $t_{\mathrm{ff}} \propto \rho^{-1 / 2}$ ) is important, assuming a constant scale height gas disc so that $\Sigma \propto \rho$. In turn, this leaves the question whether the slope of the relation is exclusively set by free-fall collapse or whether it is affected by the balance between gas heating and cooling or the balance between energy dissipation and injection by feedback.

2. Why is the molecular gas depletion time a factor of $\sim 100$ longer than the dynamical time of molecular clouds (i.e. the turbulent crossing time or gravitational free-fall time). Does this mean that star formation in clouds takes place over many dynamical times at a high integrated star formation efficiency (i.e. the integrated fraction of gas that turns into stars) or that it takes place over a single dynamical time and achieves only a low star formation efficiency? Because the normalisation of the star formation relation reflects the gas depletion time, the answer to this question will also explain the proportionality constant of the star formation relation.

While both questions are related, as are the physical mechanisms setting the star formation relation slope and normalisation, there are different ways of addressing them.

The idea that the slope of the star formation relation is set by free-fall collapse goes back decades (e.g. Madore, 1977; Krumholz et al., 2005). This interpretation has been complicated by the suggestion that the slope may vary as a function of spatial scale, going from clouds $(N \gtrsim 2$, e.g. Gutermuth et al., 2011; Lada et al., 2013) to galaxies $(N=1-1.5$, e.g. Kennicutt and Evans, 2012). The cloud-scale and galactic-scale star formation relations are not necessarily expected to be compatible, because cloud-scale relations select a single snapshot in the evolutionary timeline of molecular cloud evolution and star formation (Kruijssen et al., 2018). An additional complication is that the slope varies across the range of surface densities probed, with a steeper slope $(N \sim 2)$ at low $\left(\Sigma \lesssim 10 \mathrm{M}_{\odot} \mathrm{pc}^{-2}\right)$ and high $\left(\Sigma \gtrsim 200 \mathrm{M}_{\odot} \mathrm{pc}^{-2}\right)$ gas surface densities (e.g. Bigiel et al., 2008; Daddi et al., 2010; Genzel et al., 2010; Kennicutt and Evans, 2012), It was suggested by Krumholz et al. (2012, also see Bacchini et al. 2019) that the different incarnations of the star formation relation may be united by normalising the gas surface density to the appropriate version of the free-fall time. Specifically, if molecular clouds exhibit a large density contrast relative to the galactic midplane and thus exist in relative isolation, which happens mostly at low gas pressures, then their evolution takes place in a local free-fall time, which is much shorter than the average midplane gas free-fall time. If molecular clouds have densities similar to that of the average midplane gas, their evolution is affected by galactic dynamics and the relevant free-fall time is that of the midplane (also see Jeffreson and Kruijssen, 2018).

While these considerations address the scale dependence of the star formation relation, there is an increasing body of literature that suggests that the changing slope with surface density also has an important physical meaning. Most prominently, these studies suggest that the balance between self-gravity 
and stellar feedback sets the slope and normalisation of the star formation relation (e.g. Ostriker et al., 2010; Ostriker and Shetty, 2011; Hopkins et al., 2014; Hayward and Hopkins, 2017; Krumholz et al., 2018; Orr et al., 2018) and predict multiple physical regimes. In understanding the different regimes predicted by these models, it is important to realise that star formation is broadly speaking an accelerating process, in which the collapse time decreases as the gas contracts. This means that the slowest, rate-limiting step is presented by the first bottleneck in the evolution of the interstellar medium towards star formation. At low gas surface densities $\left(\Sigma \lesssim 10 \mathrm{M}_{\odot} \mathrm{pc}^{-2}\right)$ in the atomic gas-dominated regime the rate-limiting step is the condensation of molecular gas out of the atomic medium. Cooling competes with heating from stellar feedback, such that equating the heating and cooling rates gives:

$$
\Sigma_{\mathrm{SFR}} \propto \Sigma^{2}
$$

where the first term is the heating rate (driven by massive stars and therefore proportional to $\Sigma_{\mathrm{SFR}}$ ), the second term is the cooling rate (driven by collisions and therefore proportional to $\Sigma^{2}$ ), and we have omitted an additional dependence on metallicity (also see e.g. Schaye, 2004; Krumholz et al., 2009b,c; Ostriker et al., 2010; Hayward and Hopkins, 2017). At higher gas surface densities, the gas becomes predominantly molecular. Because the molecular gas in galaxies is supersonically turbulent, the rate-limiting step towards star formation becomes the turbulent energy dissipation rate. Equating this to the momentum injection rate by stellar feedback, we obtain:

$$
\begin{array}{rlrl}
\Sigma_{\mathrm{SFR}} & \propto \Sigma\left(\Sigma+\Sigma_{\star}\right) & \\
& \propto \Sigma & & \text { for } \Sigma \ll \Sigma_{\star} \\
& \propto \Sigma^{2} & & \text { for } \Sigma \gg \Sigma_{\star},
\end{array}
$$

where $\Sigma_{\star}$ is the stellar surface density, the first term is the momentum injection rate (driven by massive stars and therefore proportional to $\Sigma_{\mathrm{SFR}}$ ), the second term is the turbulent dissipation rate [in hydrostatic equilibrium, this is set by the motion of the gas under the influence of the total potential and is therefore proportional to $\Sigma\left(\Sigma+\Sigma_{\star}\right)$ ], and we have omitted an additional dependence on the Toomre (1964) $Q$ stability parameter (also see e.g. Ostriker and Shetty, 2011; Faucher-Giguère et al., 2013; Kim and Ostriker, 2015; Orr et al., 2018). The addition inside the parentheses implies two different regimes. At intermediate gas surface densities (lower than the stellar surface density, which in practice means $10 \lesssim \Sigma / \mathrm{M}_{\odot} \mathrm{pc}^{-2} \lesssim 100$ for star-forming main sequence galaxies in the local Universe), the star formation relation is predicted to be linear, i.e. $\Sigma_{\mathrm{SFR}} \propto \Sigma$. At high gas surface densities $\left(\Sigma \gtrsim 100 \mathrm{M}_{\odot} \mathrm{pc}^{-2}\right)$, the star formation relation is predicted to become super-linear, i.e. $\Sigma_{\mathrm{SFR}} \propto \Sigma^{2}$. These differences in slope between roughly-defined regimes of low, intermediate, and high gas surface density are in reasonable qualitative agreement with the observed star formation relation (Kennicutt and Evans, 2012).

In the equilibrium-based models discussed above, the normalisation of the star formation relation is set by the balance between the heating or momentum 
injection rate and the cooling or turbulence dissipation rate. In the molecular regime, this implies that the normalisation of the star formation relation is set by the momentum input rate per unit stellar mass, which is a single number that depends on the details of stellar evolution and the porosity of the interstellar medium (Ostriker and Shetty, 2011; Krumholz et al., 2018). More vigorous feedback drives the star formation relation to longer gas depletion times, whereas weaker feedback allows a higher SFR per unit gas mass.

While the concept of energy and momentum balance can explain the overall normalisation of the star formation relation, it does not necessarily explain whether star formation in molecular clouds takes place over many free-fall times (achieving high star formation efficiencies) or a single one (achieving a low star formation efficiency). Whichever the answer, feedback plays a key role. In the case of slow and efficient star formation, feedback would act as a homogeneous pressure term that adiabatically resists gravitational collapse, even within individual molecular clouds. In the case of rapid and inefficient star formation, feedback would act as an impulsive disruptor that disperses molecular clouds and halts the star formation process. Historically, it has been extremely challenging to measure the timescales governing the molecular cloud lifecycle, but the combination of new methodology (Kruijssen et al., 2018) and high-resolution imaging of molecular gas in nearby galaxies (e.g. Sun et al., 2018) now enables this question to be resolved. By analysing the spatial offset between tracers of molecular gas and massive star formation (e.g. Schruba et al., 2010; Kreckel et al., 2018; Schinnerer et al., 2019) it is possible to quantify the underlying cloud lifecycle. Across the local galaxy population, it is found that molecular clouds live for about a dynamical time (10-30 Myr) and achieve low star formation efficiencies (1-10\%, Kruijssen et al., 2019; Chevance et al., 2019, also see below).

The above result implies that the star formation relation is an ensemble average of the population of molecular clouds and star-forming regions, where each is individually subject to highly dynamical processes driving rapid evolutionary cycling (Kruijssen et al., 2019). Star formation in galaxies thus represents a truly multi-scale system - the cloud-scale evolution is sensitive to the large-scale energy and momentum balance, which sets e.g. the rate of cloud formation, yet the large-scale balance is also influenced by the rate and efficiency of star formation and feedback on the cloud scale, which sets the energy and momentum input rate.

\subsection{The lifecycle of molecular clouds}

Star formation in galaxies is a continuous process which is tightly coupled to the life cycle of molecular clouds. Most of the volume in galaxies is filled with hot, low density gas (Haffner et al., 2009). The mass fraction of the low-density $\left(\sim 10^{-3}-10^{-2} \mathrm{~cm}^{-3}\right)$ gas is small. However, due to the large temperatures of $\sim 10^{6} \mathrm{~K}$ this phase contains a significant fraction of the thermal energy (Ferrière, 2001). Collisional excitation and radiative cooling allow 
the hot phase to cool to $10^{4} \mathrm{~K}$ with typical densities of approximately $1 \mathrm{~cm}^{-3}$, which is the warm diffuse phase. As the cooling times in the hot phase are long compared to the dynamical time (e.g. McKee and Ostriker, 1977; Gnat and Ferland, 2012), cooling is assisted by turbulent compression. Neither the hot nor the warm gas are dominated by self-gravity and are stabilized against gravitational collapse by thermal pressure, magnetic fields and turbulent motions. We note that turbulence on the one hand creates over-densities and assist cooling and on the other hand can support the gas by an effective turbulent pressure (e.g. Mac Low and Klessen, 2004). The diffuse atomic gas can further cool down to form a colder phase with temperatures of a few $10-100 \mathrm{~K}$ with densities of $\gtrsim 10^{3} \mathrm{~cm}^{-3}$, which consists mainly of molecular rather than atomic hydrogen (Hennebelle and Falgarone, 2012; Klessen and Glover, 2016). Molecular clouds are turbulent structures with typical spatial extents of $10-50 \mathrm{pc}$ and masses ranging from $10^{2}-10^{6} M_{\odot}$ (Miville-Deschênes et al., 2017). We highlight that the cold phase and, in particular, molecular clouds and clumps are not isolated entities. Instead, they condense out of a complicated filamentary network (e.g. Arzoumanian et al., 2011). These elongated and complex filaments are in good agreement with structures that form because of the cascade of turbulent motions (e.g. Mac Low and Klessen, 2004; Hennebelle and Falgarone, 2012). We note that molecular clouds may have formed due to gravitational attraction in the galaxy, but are globally not bound by self-gravity (Heyer et al., 2009; Heyer and Dame, 2015). The virial parameter

$$
\alpha_{\mathrm{vir}}=\frac{2 T}{|W|} \approx \frac{5 \sigma_{v}^{2} R}{G M}
$$

relates the kinetic $(T)$ to the gravitational energy $(W)$ of a cloud. Assuming a homogeneous sphere this ratio can be approximated as the last term where $\sigma_{v}$ is the one-dimensional velocity dispersion, $R$ is the radius, $M$ is the mass, and $G$ the gravitational constant. Observed values of $\alpha_{\text {vir }}$ for clouds in the Milky Way span two orders of magnitude from $1-100$ (Miville-Deschênes et al., 2017). However, we note that there is no consensus on how important gravity is for cloud formation and dynamical driving. As the warm and the cold phase can coexist in pressure equilibrium (Field, 1965; McKee and Ostriker, 1977), turbulent motions and gravity play an important role in shaping molecular clouds.

The next step towards star formation is the gravitational instability that together with turbulence, rotation and magnetic fields - determines the fragmentation of gas and the resulting spatial and mass distribution of collapsing clumps that are the sites of star and star cluster formation. Once fragmented regions start to locally collapse due to self-gravity, the opposing forces like thermal pressure and magnetic fields become less significant and the starforming process can be described as a gravo-turbulent process. During the fragmentation and contraction of the molecular cloud it is also very likely that many individual regions collapse simultaneously. As a result, cores and stars typically form in groups, i.e. clusters and associations, (Lada and Lada, 2003; Bressert et al., 2010; Kruijssen, 2012). However, the fraction of how many stars 
form in clusters and details of the clustering are still debated (e.g. Longmore et al., 2014; Krumholz et al., 2019; Ward et al., 2019). The first gravitational instability only sets the seeds of star formation. The actual masses of stars are further influenced by accretion from the ambient reservoir. In the case of high-mass star formation two extreme models have been proposed and found in numerical simulations. In the competitive accretion model (Bonnell et al., 2001) gas is funneled into the centre of the gravitational potential. Accretion onto centrally located stars is then favoured, which allows them to grow into the most massive stars. The opposite effect occurs in the case of fragmentation induced starvation, where gas on its way to the centre of the cluster fragments (Peters et al., 2010a; Girichidis et al., 2012; Kruijssen et al., 2012). The new stars accrete in-flowing material, thereby depriving the central stars of material and halting their runaway growth. Which model is favoured depends on the details of the accretion flow, the nature of the turbulence, the available gas in the vicinity of the local gravitational centre, the position of the collapsing regions with respect to neighbouring condensations or the position relative to the centre of the molecular cloud core (Girichidis et al., 2011), and, finally, the onset of stellar activity and early (proto) stellar feedback, which can halt accretion (Peters et al., 2010b; Geen et al., 2015). The multitude of all of these processes eventually produces a remarkably universal distribution of stellar masses (Kroupa, 2001; Chabrier, 2003). The stellar initial mass function can be described by a lognormal distribution around a peak at $\sim 0.2 \mathrm{M}_{\odot}$ and a high-mass power law with a slope of $d N / d M \propto M^{-2.3}$ (Salpeter, 1955). However, simulations predict the mass function of the first stars in the early universe is a notable exception, since the lack of metal coolants changes the fragmentation behaviour and is likely to result in a more top-heavy IMF, i.e. an overabundance in massive stars compared to the present-day IFM (Greif et al., 2011; Clark et al., 2011; Stacy and Bromm, 2013; Susa, 2013; Hirano et al., 2014; Stacy et al., 2016).

There are several forms of stellar feedback: protostellar outflows, radiation, stellar winds, and supernovae, where the latter two processes are mainly relevant for massive stars. The combined interaction of stellar feedback keeps the integrated star-formation efficiency low, at a few percent, and eventually leads to the dissolution of molecular clouds after about a dynamical time (1030 Myr, see e.g. Walch et al., 2012; Dale et al., 2013; Kruijssen et al., 2019; Chevance et al., 2019). A fraction of the gas can be pushed out of the galactic disc to form a fountain flow or even escape from the galaxy as a wind (e.g. Hill et al., 2012; Walch et al., 2015; Girichidis et al., 2016). With mass outflow rates that can be comparable to the star formation rate these outflows have a strong dynamical impact on the evolution of galaxies and the redistribution of gas in the ISM (see e.g. reviews by Veilleux et al., 2005; Somerville and Davé, 2015; Naab and Ostriker, 2017). The turbulent hot gas, which is enriched with metals produced in the stars then forms the reservoir from which the next cycle of molecular clouds forms. It is important to note that the cycle of gas in the interstellar medium is not a truly periodic cycle but rather a continuous 
process, in which different regions of the interstellar medium pass through the hot, warm and cold phases at different rates.

One important aspect of stellar feedback is that it is not only disruptive. Instead, pressure waves, ejected material and turbulence created by feedback can also locally trigger the onset of gravitational collapse and thereby cause star formation by increasing the gas density and accelerating cooling, a result known as triggered star formation.

\section{Composition and thermodynamic behavior of the ISM}

The interstellar medium is composed almost entirely of hydrogen and helium, with the former accounting for around $70 \%$ of the total mass and the latter for $28 \%$. All other elements contribute the remaining $2 \%$ (Draine, 2011). While most of the light elements are found in the gas phase (Section 2.1), a large fraction of the heavier elements can be locked up in dust grains, corresponding on average to about $1 \%$ of the total mass (see $\S 2.2$ ). We provide an overview of the most important chemical reactions in $\S 2.3$ and of the relevant cooling and heating processes in $\S 2.4$ and $\S 2.5$.

The total gas mass in the Milky Way is difficult to estimate but is probably close to $10^{10} \mathrm{M}_{\odot}$ (Kalberla and Kerp, 2009). The majority of the volume of the ISM is occupied by ionized gas, but the total mass associated with this component is not more than around $25 \%$ of the total gas mass. The majority of the mass is located in regions dominated by neutral atomic gas $(\mathrm{H}, \mathrm{He})$ or molecular gas $\left(\mathrm{H}_{2}\right)$. Much of the atomic gas and all of the molecular gas is found in the form of dense clouds that occupy only $1-2 \%$ of the total ISM volume (see e.g. Ferrière, 2001).

\subsection{Different gas phases}

The changes in the chemical make-up of the ISM also go hand in hand with different thermal phases as discuss in more detail in Section 3. Starting with atomic gas, often called warm neutral medium (in short WNM), the main transitions are collisional ionization, leading to the warm ionized medium (WIM) when the temperature exceeds $10^{4} \mathrm{~K}$, or ionization due to ultraviolet photons in the vicinity of high-mass stars, creating classical HII regions. Similarly, the formation of the hydrogen molecule marks the build-up of molecular clouds with densities above $\sim 100 \mathrm{~cm}^{-3}$ and temperatures below $\sim 100 \mathrm{~K}$.

The simplest model of the ISM phase structure was suggested by Field et al. (1969). If one assumes that the atomic gas in the ISM is in thermal equilibrium and in pressure balance, then there are two thermally stable solutions for a wide range of pressures. The cold dense phase corresponds to the cold neutral medium (CNM) introduced earlier, and the warm, diffuse phase is the warm neutral medium (WNM) discussed above. In the Field et al. (1969) model, gas at intermediate temperatures is thermally unstable: depending on its density 
it will either cool and get denser until it joins the CNM, or heat and become more tenuous until it joins the WNM. At high densities, there is considerable overlap between the physical parameters of the CNM and of molecular clouds, which are often thought to a have an envelope of dense neutral atomic gas. This two-phase model was extended by McKee and Ostriker (1977), who pointed out that supernovae exploding in the ISM would create large, ionized bubbles filled with very hot gas $\left(T \sim 10^{6} \mathrm{~K}\right)$. Although this gas would eventually cool, the temperature dependence of the atomic cooling curve at high temperatures is such that the cooling time around $T \sim 10^{6} \mathrm{~K}$ is considerably longer than the cooling time in the temperature range $10^{4} \mathrm{~K}<T<10^{6} \mathrm{~K}$ (see $\S 2.3$ below). It is also longer than the time needed for supernovae to produce appreciable amounts of ionized material. And so, while gas at the low end of the temperature range quickly cools to join the WIM, gas close to $\sim 10^{6} \mathrm{~K}$ remains at these temperatures, effectively forming a third phase known as the hot ionized medium (HIM).

The Galactic distribution of molecular gas can be estimated by combining data from $\mathrm{CO}$ observations, which trace clouds with high concentrations of both $\mathrm{H}_{2}$ and $\mathrm{CO}$, with measurements of $\mathrm{C}^{+}$, which trace so-called "dark molecular gas", i.e. clouds with high $\mathrm{H}_{2}$ fractions but little $\mathrm{CO}$ (see e.g. Pineda et al., 2013). The molecular gas surface density shows a peak within the central few hundred parsec of the Galaxy, a region known as the Central Molecular Zone (CMZ). It then falls off sharply between 0.5 and $3 \mathrm{kpc}$, possibly owing to the influence of the Milky Way's central stellar bar (Morris and Serabyn, 1996), before peaking again at a Galactocentric radius of around 4-6 kpc in a structure known as the Molecular Ring. Outside the Molecular Ring, the surface density of molecular gas declines exponentially, but it can still be traced out to distances of at least 12-13 kpc (Heyer et al., 1998).

Observational evidence for the presence of the WIM comes from free-free absorption of the Galactic synchrotron background (Hoyle and Ellis, 1963), the dispersion of radio signals from pulsars (Reynolds, 1989; Gaensler et al., 2008), and collisionally excited faint optical emission lines produced by ionized species such as $\mathrm{O}^{+}$and $\mathrm{N}^{+}$(Reynolds et al., 1973; Mierkiewicz et al., 2006). Its density is comparable to the WNM with a scale-height of the order of $1 \mathrm{kpc}$ (see e.g. Reynolds, 1989). It is thought that over $90 \%$ of the total ionized gas within the ISM is located in the WIM (Haffner et al., 2009). We note that the ionized gas in classical HII regions surrounding O stars is generally not considered to be part of the WIM. As mentioned above, the material in the WIM is collisionally ionized, whereas the high ionization degree in classical HII regions is due to ultraviolet photons from massive stars. We summarize the main physical properties of the different ISM phases in Table 1. We note, however, that the picture of a simple three-phase medium is a severe over-simplification for many real applications. In many regions of the galaxy, these phases appear strongly intermixed. This is due to the fact that the ISM is a highly turbulent medium. Turbulence in the ISM is driven by a number of different physical processes, including thermal instability (Section 3.2), supernova feedback (see e.g. Mac Low and Klessen, 2004; Klessen and Glover, 2016), and the inflow 
of gas onto the disk (Klessen and Hennebelle, 2010; Elmegreen and Burkert, 2010). It acts to mix what would otherwise be distinct ISM phases (see e.g. Joung et al., 2009; Seifried et al., 2011). We discuss the role that turbulence plays in structuring the ISM in $\S 4$.

Table 1 Phases of the ISM

\begin{tabular}{rrrr}
\hline Component & $\begin{array}{r}\text { Temperature } \\
(\mathrm{K})\end{array}$ & $\begin{array}{r}\text { Density } \\
\left(\mathrm{cm}^{-3}\right)\end{array}$ & $\begin{array}{r}\text { Fractional } \\
\text { ionization }\end{array}$ \\
\hline $\begin{array}{r}\text { Molecular gas } \\
10-20\end{array}$ & $>10^{2}$ & $<10^{-6}$ \\
Cold neutral medium (CNM) & $50-100$ & $20-50$ & $\sim 10^{-4}$ \\
Warm neutral medium (WNM) & $6000-10000$ & $0.2-0.5$ & $\sim 0.1$ \\
Warm ionized medium (WIM) & $\sim 8000$ & $0.2-0.5$ & 1.0 \\
Hot ionized medium (HIM) & $\sim 10^{6}$ & $\sim 10^{-2}$ & 1.0 \\
\hline
\end{tabular}

Adapted from Ferrière (2001), Caselli et al. (1998), Wolfire et al. (2003), and Jenkins

(2013).

\subsection{Interstellar dust}

The reddening of starlight in the ISM points towards an additional component, responsible for absorbing light over a wide range of frequencies. There are distinct features in the extinction curve, for example the $217.5 \mathrm{~nm}$ bump that tend to be extremely broad, very different from the narrow lines and structured bands that we expect from atoms or small molecules. Furthermore, abundance measurements show that a number of elements, notably silicon and iron, are strongly depleted in the gas-phase when compared to the solar value. These are typically also the most refractory elements Draine (2011). Finally, midinfrared and far-infrared observations show widespread continuum emission, with a spectrum close to that of a blackbody, and an intensity that correlates well with the hydrogen column density. We conclude that besides the ionized, atomic and molecular constituents of the ISM there is an addition component, which we usually call dust.

We gain further insight into the nature of dust by looking at the spectral shape of the extinction curve that it produces. To first approximation, individual dust grains absorb only those photons with wavelengths smaller than the physical size of the grain. From the fact that we see a large amount of absorption in the ultraviolet, somewhat less in the optical and even less at infrared wavelengths, we can directly infer that there are many more small dust grains than there are large ones. In addition, we often associate particular spectral features in the extinction curve with particular types of dust grain, e.g. graphite in the case of the $217.5 \mathrm{~nm}$ bump (Mathis et al., 1977) and amorphous silicates in the case of the infrared bands at $9.7 \mu \mathrm{m}$ and $18 \mu \mathrm{m}$ (e.g. Draine and Lee, 1984; Draine and Li, 2007). 
This can be used to derive more quantitative constraints on the size distribution of interstellar dust grains. For example, Mathis et al. (1977) reproduced the ISM extinction curve between $0.1-1 \mu \mathrm{m}$ with a mixture of spherical graphite and silicate grains with a size distribution

$$
N(a) d a \propto a^{-3.5} d a,
$$

where $a$ is the grain radius and the distribution extends over a range of radii from $a_{\text {min }} \sim 50 \mathrm{~nm}$ to $a_{\max } \sim 0.25 \mu \mathrm{m}$. We note however that actual grains are not spherical, as evidenced by polarization of starlight in extinction and of thermal emission in the submillimetre. Subsequent studies have improved on this simple description (see e.g. Draine and Lee, 1984; Weingartner and Draine, 2001a), but it remains a useful first approximation to the properties of interstellar dust. Due to the steep distribution of grain sizes, see (4), all models predict that the total mass is dominated by the contribution from large grains, while the total surface area is dominated by the contribution made by small grains (for a more detailed discussion, see Draine, 2011).

The total mass in dust grains is difficult to constrain, but if we combine absorption measurements with the observed elemental depletion patterns in the cold ISM, we find that the total mass of metals locked up in grains is roughly the same as the total mass of metals in the gas phase. The dust therefore accounts for around 1\% of the total ISM mass. Therefore, when we attempt to model the thermal and chemical behavior of the ISM dust can play a role that is as important or more important than the gas-phase metals.

\subsection{Chemistry of molecular cloud formation}

As star formation in the local universe takes place in molecular clouds, we focus our attention now on the chemical processes that lead to the build-up of these clouds. We note that there are two main chemical transitions, occurring at different points in their assembly history, which we can use to identify molecular clouds. The first and most fundamental of these is the transition from atomic to molecular hydrogen. We define a molecular cloud as a region where most of the hydrogen content is in the form of $\mathrm{H}_{2}$ rather than $\mathrm{H}$. However, due to the symmetric structure of the $\mathrm{H}_{2}$ molecule has no electric dipole transitions but only (forbidden) higher-order ones, and so it does not emit at the temperatures of a few $10 \mathrm{~K}$ to $100 \mathrm{~K}$ typical of Galactic clouds. Therefore, it is common to use a different, observationally-motivated definition, which refers to the moment when the cloud becomes visible in $\mathrm{CO}$ emission. This requires understanding the chemical transition from $\mathrm{C}^{+}$to $\mathrm{C}$ to $\mathrm{CO}$ within the assembling cloud, which we discuss in $§ 2.3 .2$ ).

\subsubsection{Transition from $\mathrm{H}$ to $\mathrm{H}_{2}$}

The simplest way to form $\mathrm{H}_{2}$ in the ISM is via the radiative association of two hydrogen atoms, i.e.

$$
\mathrm{H}+\mathrm{H} \rightarrow \mathrm{H}_{2}+\gamma \text {. }
$$


However, the rate coefficient for this reaction is extremely small (Latter and Black, 1991), so that very little $\mathrm{H}_{2}$ forms in this way. Ion-neutral reaction pathways

$$
\begin{gathered}
\mathrm{H}+\mathrm{e}^{-} \rightarrow \mathrm{H}^{-}+\gamma, \\
\mathrm{H}^{-}+\mathrm{H} \rightarrow \mathrm{H}_{2}+\mathrm{e}^{-},
\end{gathered}
$$

and

$$
\begin{aligned}
& \mathrm{H}+\mathrm{H}^{+} \rightarrow \mathrm{H}_{2}^{+}+\gamma, \\
& \mathrm{H}_{2}^{+}+\mathrm{H} \rightarrow \mathrm{H}_{2}+\mathrm{H}^{+},
\end{aligned}
$$

are also highly inefficient. And so it is difficult to produce a $\mathrm{H}_{2}$ fractional abundances larger than $f_{\mathrm{H}_{2}} \sim 10^{-2}$ with these reactions, even in the most optimal conditions (see e.g. Tegmark et al., 1997). Moreover, photodetachment of $\mathrm{H}^{-}$ and photodissociation of $\mathrm{H}_{2}^{+}$by photons from the interstellar radiation field render these pathways considerably less effective (Glover, 2003). Consequently, the formation of $\mathrm{H}_{2}$ in the gas phase is extremely inefficient under typical ISM conditions.

Formation of $\mathrm{H}_{2}$ in the Milky Way and in essentially all galaxies in the low-redshift Universe therefore follows a very different pathway: molecular hydrogen assembles on the surface of dust grains (Gould and Salpeter, 1963). This is an exothermic reaction with the energy released being absorbed in part by the dust grain and in part being converted to internal energy and kinetic energy of the $\mathrm{H}_{2}$ molecule (see e.g. Bron et al., 2014). Association reactions between adsorbed hydrogen atoms occur readily on grain surfaces; the rate at which $\mathrm{H}_{2}$ forms is then limited primarily by the rate at which $\mathrm{H}$ atoms are adsorbed onto the surface. For typical Milky Way conditions, the resulting $\mathrm{H}_{2}$ formation rate is approximately (Jura, 1975)

$$
R_{\mathrm{H}_{2}} \sim 3 \times 10^{-17} n n_{\mathrm{H}} \mathrm{s}^{-1} \mathrm{~cm}^{-3} .
$$

Here, $n$ is the total number density of gas particles, while $n_{\mathrm{H}}$ is the number density of atomic hydrogen, all given in units of particles per $\mathrm{cm}^{3}$. For atomic hydrogen gas, both quantities are identical if we neglect contributions from helium and possibly metals. Note that $n_{\mathrm{H}}$ declines as the molecular fraction increases, while $n$ remains the same in the absence of compression or expansion. The $\mathrm{H}_{2}$ formation timescale corresponding to the formation rate (10) is approximately

$$
t_{\text {form }}=\frac{n_{\mathrm{H}}}{R_{\mathrm{H}_{2}}} \sim 10^{9} n^{-1} \mathrm{yr} .
$$

We point out that at low density this timescale is considerably longer than the dynamical timescale of the system, such as the gravitational free-fall time or the turbulent crossing time. In order to form molecular clouds within a timescale of several million years as inferred by observations (Fukui et al., 2001; Clark and Glover, 2014), we must again invoke interstellar turbulence. The intermittent compression of gas due to supersonic turbulent gas motions 
can shorten $t_{\text {form }}$ considerably (see e.g. Glover and Mac Low, 2007; Micic et al., 2012).

Complementary to the processes that lead to $\mathrm{H}_{2}$ formation, we must also consider $\mathrm{H}_{2}$ destruction. Destruction can happen by collision with another atom or molecule in the cloud or by ultraviolet photodissociation. Focusing on the main cloud constituents, collisional dissociation can be formulated as

$$
\begin{aligned}
\mathrm{H}_{2}+\mathrm{H} & \rightarrow \mathrm{H}+\mathrm{H}+\mathrm{H}, \\
\mathrm{H}_{2}+\mathrm{H}_{2} & \rightarrow \mathrm{H}+\mathrm{H}+\mathrm{H}_{2} .
\end{aligned}
$$

We note that these reactions do not play an important role in regulating the molecular content of the ISM. They are only effective at destroying $\mathrm{H}_{2}$ in warm, dense gas, such as observed in molecular outflows (see e.g. Flower et al., 2003). Consequently, the key reaction for our purpose here is photodissociation of $\mathrm{H}_{2}$, which occurs via a process known as spontaneous radiative dissociation (Stecher and Williams, 1967; van Dishoeck, 1987). The $\mathrm{H}_{2}$ molecule first absorbs a UV photon with energy $E>11.2 \mathrm{eV}$, placing it in an excited electronic state. The excited $\mathrm{H}_{2}$ molecule then undergoes a radiative transition back to the electronic ground state. This transition can occur either into a bound rovibrational level in the ground state, in which case the molecule survives, or into the vibrational continuum, in which case it dissociates. Altogether the dissociation probability is around $15 \%$ per UV photon absorption (Draine and Bertoldi, 1996). The decay back to the rovibrational ground state produces a discrete set of UV absorption lines which are known as the Lyman-Werner bands.

Because $\mathrm{H}_{2}$ photodissociation is line-based, rather than continuum-based, the $\mathrm{H}_{2}$ photodissociation rate in the ISM is highly sensitive to self-shielding. Lyman-Werner photons of the ambient radiation field with energies corresponding to the main absorption lines are mostly absorbed by $\mathrm{H}_{2}$ on the surface of the molecular cloud, with only a few photons remaining in the regions further in. Consequently, the $\mathrm{H}_{2}$ photodissociation rate drops by a large factor compared to the rate in the unshielded, optically thin gas. This becomes important once the $\mathrm{H}_{2}$ column density exceeds $N_{\mathrm{H}_{2}} \sim 10^{14} \mathrm{~cm}^{-2}$ (Draine and Bertoldi, 1996). The total column density of hydrogen, $N$, depends on the strength of the interstellar radiation field and on the density $n$ of the gas. When we express the radiation field in Habing units $G_{0}$ (Habing, 1968) we find that self-shielding becomes important for column densities exceeding a value of

$$
N=10^{20} G_{0} n^{-1} \mathrm{~cm}^{-2} .
$$

We note that the visual extinction required to reduce the $\mathrm{H}_{2}$ photodissociation rate by a factor of ten is approximately $A_{\mathrm{V}} \approx 0.65$. In the diffuse ISM this corresponds to a total hydrogen column density $N \sim 10^{21} \mathrm{~cm}^{-2}$. Consequently, $\mathrm{H}_{2}$ self-shielding becomes important at lower total column densities than dust shielding in conditions when $G_{0} / n$ is small, such as in the CNM far away from regions of massive star formation. On the other hand, if $G_{0} / n$ is large, e.g. in the photodissociation regions surrounding massive stars dust extinction typically dominates. 


\subsubsection{Transition from $C^{+}$to $C$ to $C O$}

The chemistry involved in the transition from $\mathrm{C}^{+}$to $\mathrm{C}$ is very simple. Atomic carbon forms via the radiative recombination of $\mathrm{C}^{+}$,

$$
\mathrm{C}^{+}+\mathrm{e}^{-} \rightarrow \mathrm{C}+\gamma
$$

and is destroyed by photoionization,

$$
\mathrm{C}+\gamma \rightarrow \mathrm{C}^{+}+\mathrm{e}^{-}
$$

The formation of CO is considerably more complicated, because there is no single dominant process but instead a number of different pathways compete to build up $\mathrm{CO}$. The two main routes to $\mathrm{CO}$ formation involve either hydroxyl $(\mathrm{OH})$ or its positive ion $\left(\mathrm{OH}^{+}\right)$as chemical intermediates, or they rely on simple hydrocarbons such as $\mathrm{CH}$ or $\mathrm{CH}_{2}$ and their positive ions. A brief summary of these reaction rates is provided by Klessen and Glover (2016). For more details and a more comprehensive discussion we refer the readers to the classic papers by Glassgold and Langer (1975), Langer (1976), Dalgarno and Black (1976), Tielens and Hollenbach (1985) and Sternberg and Dalgarno (1995).

Both main CO-formation pathways share one key feature. The rate-limiting step is the formation of the initial chemical intermediate. In the first case, it is the build-up of $\mathrm{OH}$ and the $\mathrm{OH}^{+}$ion. In the second case, it is the formation of $\mathrm{CH}, \mathrm{CH}_{2}, \mathrm{CH}^{+}$or $\mathrm{CH}_{2}^{+}$by radiative association or the formation of $\mathrm{H}_{3}^{+}$as a consequence of the cosmic ray ionization of $\mathrm{H}_{2}$. Once the initial molecular ion or radical forms, the remainder of the reactions that lead to $\mathrm{CO}$ proceed relatively quickly. This behavior forms the basis of several simplified methods for treating CO formation (for a comparison of different approaches, see Glover and Clark, 2012a). In addition, all of the different pathways to proceed from $\mathrm{C}^{+}$or $\mathrm{C}$ to $\mathrm{CO}$ rely on the presence of molecular hydrogen. It implies that substantial quantities of $\mathrm{CO}$ will form only in regions that already have high $\mathrm{H}_{2}$ fractions. As a consequence, the non-equilibrium, time-dependent behavior of $\mathrm{H}_{2}$ chemistry can be important also for $\mathrm{CO}$, despite the fact that the characteristic timescales of the chemical reactions involved in $\mathrm{CO}$ formation are generally shorter than the $\mathrm{H}_{2}$ formation time.

In the surface layers of molecular clouds, i.e. regions with a low visual extinction, the destruction of $\mathrm{CO}$ is dominated by photodissociation,

$$
\mathrm{CO}+\gamma \rightarrow \mathrm{C}+\mathrm{O}
$$

The molecule first absorbs a UV photon with energy $E>11.09 \mathrm{eV}$, placing it in an excited electronic state (van Dishoeck, 1987). From here, it can either return to the ground state via radiative decay, or it can undergo a transition to a repulsive electronic state via a radiationless process. In the latter case, the molecule very rapidly dissociates. In the case of $\mathrm{CO}$, dissociation is typically far more likely than decay back to the ground state (van Dishoeck and Black, 1988; Visser et al., 2009). Consequently, the lifetimes of the excited electronic states are very short. This is important, as Heisenberg's uncertainty principle 
then implies that their energy is comparatively uncertain. The UV absorption lines associated with the photodissociation of $\mathrm{CO}$ are therefore much broader than the lines associated with $\mathrm{H}_{2}$ photodissociation. As a result, more photons need to be absorbed to prevent molecules further into the cloud from being photodissociated, and consequently $\mathrm{CO}$ self-shielding is less effective than the analogous process for $\mathrm{H}_{2}$ discussed above.

Once the visual extinction $A_{\mathrm{V}}$ at the cloud surface due to dust absorption and $\mathrm{H}_{2}$ shielding becomes large, very few photons remain in the cloud interior to photodissociate $\mathrm{CO}$, and so two other processes become important in balancing CO formation. First, cosmic ray ionization of hydrogen molecules or hydrogen atoms produces energetic photo-electrons (see Section 2.5.3). If these collide with other hydrogen molecules before dissipating their energy, they can excite the $\mathrm{H}_{2}$ molecules into excited electronic states. The subsequent radiative decay of the molecules back to the ground state produces UV photons that can initiate localized photodissociation of CO and other molecules (Prasad and Tarafdar, 1983; Gredel et al., 1987, 1989). Second, CO is also destroyed via dissociative charge transfer with $\mathrm{He}^{+}$ions

$$
\mathrm{CO}+\mathrm{He}^{+} \rightarrow \mathrm{C}^{+}+\mathrm{O}+\mathrm{He} .
$$

The $\mathrm{He}^{+}$ions required by this reaction are again produced by cosmic ray ionization of neutral helium. Altogether, $\mathrm{CO}$ destruction in high $A_{\mathrm{V}}$ regions is controlled by the cosmic ray ionization rate. This is relatively small (van der Tak and van Dishoeck, 2000) in typical clouds, and so almost all of the carbon is found in the form of CO. The situation changes in regions of high cosmic ray flux, such as inferred for the Central Molecular Zone of the Galaxy, where the CO fraction can be significantly suppressed even in well-shielded gas (see e.g. Clark et al., 2013).

\subsection{Cooling processes}

In this section we summarize the key heating and cooling processes that determine the thermal evolution of the ISM. Most cooling processes are based on the fact that collisions can excite internal degrees of freedom of the atom, molecule, ion or dust grain under consideration, therefore removing kinetic energy from the system, and that there are de-excitation pathways that involve the emission of photons. If these photons are absorbed nearby i.e. there are optically thick conditions, the energy remains in the system and there is no net cooling effect. If the photons leave the system the medium is optically thin, and their energy is carried away and the system is cooler. We note that also adiabatic expansion can lead to a decrease of the kinetic temperature of the medium. Most heating processes are based on the inverse of these processes. However, we note that reality can be more complicated due to the fact that some chemical phase changes involve latent heat, which needs to be included in the energy budget. There are other potential heating and cooling mechanisms that are not based on collisional or radiative coupling or on cloud dynamics 
but instead may involve interaction with a magnetic field to exchange energy, such as heating by magnetic reconnection.

For the microphysical treatment of the collisional and radiative coupling of multi-level atoms and molecules, we refer to the relevant textbooks, e.g. to Rybicki and Lightman (1986), Osterbrock (1989), Tielens (2010), and Draine (2011), or to a recent compilation provided by Klessen and Glover (2016).

\subsubsection{Cooling by permitted transitions}

At high temperatures in regions dominated by atomic or ionized gas, the cooling of the ISM takes place largely via permitted (i.e. dipole-allowed) electronic transitions of various atoms and ions. At temperatures close to $10^{4} \mathrm{~K}$, excitation of the Lyman series lines of atomic hydrogen is the dominant process leading to a cooling rate per unit volume (Black, 1981; Cen, 1992) of

$$
\Lambda_{\mathrm{H}}=7.5 \times 10^{-19} \mathrm{erg} \mathrm{s}^{-1} \mathrm{~cm}^{-3} \frac{1}{1+\left(T / 10^{5}\right)^{1 / 2}} \exp \left(\frac{-118348}{T}\right) n_{\mathrm{e}} n_{\mathrm{H}},
$$

where $n_{\mathrm{e}}$ and $n_{\mathrm{H}}$ are the number densities of free electrons and atomic hydrogen given in units of $\mathrm{cm}^{-3}$, respectively. At temperatures $T \sim 3 \times 10^{4} \mathrm{~K}$ and above, however, the abundance of atomic hydrogen generally becomes very small, and other elements, particularly $\mathrm{C}, \mathrm{O}, \mathrm{Ne}$ and $\mathrm{Fe}$, start to dominate the cooling (see e.g. Gnat and Ferland, 2012).

We note that there are many cases in which the assumption of collisional ionization equilibrium does not apply. For example, consider gas in the HII regions around massive stars, where the ionization state of the gas is determined primarily by photoionization rather than collisional ionization. The assumption of collisional ionization equlibrium also breaks down whenever the gas cools rapidly, i.e. when the cooling time becomes shorter than the recombination time, or if gas is heated more rapidly than it is collisionally ionized, such as in a very strong shock. There are many efforts to account for non-equilibrium effects, either by explicitly solving for the non-equilibrium ionization state (see e.g. Cen and Fang, 2006; de Avillez and Breitschwerdt, 2012; Oppenheimer and Schaye, 2013; Richings et al., 2014) or with an ionization state dominated by photoionization rather than collisional ionization (e.g. Wiersma et al., 2009; Gnedin and Hollon, 2012).

\subsubsection{Cooling by fine structure lines}

At temperatures far below $10^{4} \mathrm{~K}$, it becomes extremely difficult for the gas to cool via radiation from permitted atomic transitions, as the number of electrons available with sufficient energy to excite these transitions declines exponentially with decreasing temperature. In this regime forbidden transitions between different fine structure energy levels become important.

Fine structure splitting is caused by the coupling between the orbital and spin angular momenta of the electrons in an atom (see e.g. Atkins and Friedman, 2011). Each electron within an atom has a magnetic moment due to its 
orbital motion and also an intrinsic magnetic moment due to its spin. States where these magnetic moments are parallel have higher energy than states where they are anti-parallel. In order for an atom or ion to display fine structure splitting in its ground state, the electrons in the outermost shell must have both non-zero total orbital angular momentum (i.e. $L>0$ ) and non-zero total spin angular momentum (i.e. $S>0$ ), or else the spin-orbit coupling term in the Hamiltonian, which is proportional to $\mathbf{L} \cdot \mathbf{S}$, will vanish. The resulting splitting of the energy levels is small, with energy separations of the order of $10^{-2} \mathrm{eV}$. This corresponds to a temperature of the order of $100 \mathrm{~K}$, meaning that it is possible to excite these transitions even at relatively low gas temperatures.

The corresponding quantum mechanical transition matrix elements are of the order of $\alpha^{2} \approx 5 \times 10^{-5}$ times smaller than for electric dipole transitions, where $\alpha$ is the fine structure constant. Furthermore, the spontaneous transition rates scale as $A_{i j} \propto \nu_{i j}^{3}$. Since associated frequencies are about a thousand times smaller than those of the most important permitted electronic transitions, such as Lyman- $\alpha$, one expects the spontaneous transition rates to be a factor of $10^{9}$ smaller. These two effects combined result in transition rates that are of order of $10^{14}$ times smaller than those of the permitted atomic transitions. Consequently, the critical densities associated with many of the important fine structure lines are relatively low: $n_{\text {crit }} \sim 10^{2}-10^{6} \mathrm{~cm}^{-3}$ in conditions when collisions with $\mathrm{H}$ or $\mathrm{H}_{2}$ dominate, and up to two to three orders of magnitude smaller when collisions with electrons dominate (Hollenbach and McKee, 1989). Therefore fine structure emission is effective in the WNM or CNM but not so important at the much higher densities found in gravitationally collapsing regions within molecular clouds.

Since hydrogen and helium have no fine structure in their ground states, fine structure cooling in the diffuse ISM is dominated by the contribution from the next most abundant elements: carbon and oxygen in their atomic and low ionization states (Wolfire et al., 1995). Data on the collisional excitation rates of the fine structure transitions of $\mathrm{C}^{+}, \mathrm{C}$ and $\mathrm{O}$ can be found in a number of places in the literature. Compilations of excitation rate data are given in Hollenbach and McKee (1989), Glover and Jappsen (2007) and Maio et al. (2007), as well as in the LAMDA database (Schöier et al., 2005).

\subsubsection{Carbon monoxide}

In order to study cold and dense molecular clouds, we need to resort to lowenergy rotational and vibrational transitions of molecular species. Molecular hydrogen is a symmetric molecule and therefore has no permanent dipole moment. The next order transition based on the quadrupole moment requires higher temperatures than are usually found in Galactic molecular clouds and are typically not observed. Luckily, the second most abundant molecular species, CO, has a significant dipole moment. It is readily rotationally excited even at very low gas temperatures, $T<20 \mathrm{~K}$, owing to very small energy 
separations between its excited rotational levels. It therefore plays a key role in the thermal balance of cold, dense clouds.

We note that at low densities, fine structure cooling from neutral atomic carbon is more effective than $\mathrm{CO}$ cooling, and that at $T \sim 20 \mathrm{~K}$ and above the contribution from $\mathrm{C}^{+}$also becomes significant. The overall importance of $\mathrm{CO}$ therefore depends strongly on the chemical state of the gas. If the gasphase carbon is primarily in the form of $\mathrm{C}$ or $\mathrm{C}^{+}$, then fine structure emission from these species will dominate, implying that $\mathrm{CO}$ becomes important only once the fraction of carbon in $\mathrm{CO}$ becomes large. Consequently, CO cooling only dominates once the gas density exceeds $n \sim 1000 \mathrm{~cm}^{-3}$. Furthermore, the relevant density range is quite limited. For $n \gg 1000 \mathrm{~cm}^{-3}$ the relative populations of the lowest rotational levels quickly reach their LTE levels. Furthermore, the lines of the most abundant species, ${ }^{12} \mathrm{CO}$ become optically thick and the molecule tends to freeze out on dust grains and its gas phase abundance drops significantly (Goldsmith, 2001).

\subsubsection{Gas-grain energy transfer}

At high temperatures dust can also play an important role in the cooling of the ISM (Goldreich and Kwan, 1974; Leung, 1975). Individual dust grains are extremely efficient radiators, and so the mean temperature of a population of dust grains very quickly relaxes to an equilibrium value given by the balance between radiative heating caused by absorption of photons from the interstellar radiation field (ISRF) and radiative cooling via thermal emission from the grains. If the resulting dust temperature, $T_{\mathrm{d}}$, differs from the gas temperature, $T_{\mathrm{K}}$, then collisions between gas particles and dust grains lead to a net flow of energy from one component to the other, potentially changing both $T_{\mathrm{K}}$ and $T_{\mathrm{d}}$.

We can write the cooling rate per unit volume due to energy transfer from the gas to the dust as

$$
\Lambda_{\mathrm{gd}}=\pi \sigma_{\mathrm{d}} \bar{v} \bar{\alpha}\left(2 k T_{\mathrm{K}}-2 k T_{\mathrm{d}}\right) n_{\mathrm{tot}} n_{\mathrm{d}} .
$$

Here, $\sigma_{\mathrm{d}}$ is the mean cross-sectional area of a dust grain, $n_{\mathrm{d}}$ is the number density of dust grains, $n_{\text {tot }}$ is the number density of particles, and $\bar{v}$ is the mean thermal velocity of the particles in the gas. Although it is common to discuss this in terms of cooling, if $T_{\mathrm{d}}>T_{\mathrm{K}}$ then energy will flow from the dust to the gas, i.e. this will become a heating rate. $\Lambda_{\mathrm{gd}}$ is often expressed in the form

$$
\Lambda_{\mathrm{gd}}=C_{\mathrm{gd}} T_{\mathrm{K}}^{1 / 2}\left(T_{\mathrm{K}}-T_{\mathrm{d}}\right) n^{2} \mathrm{erg} \mathrm{s}^{-1} \mathrm{~cm}^{-3},
$$

where $n$ is the number density of hydrogen nuclei and $C_{\mathrm{gd}}$ is a cooling rate coefficient given by

$$
C_{\mathrm{gd}}=2 \pi k \sigma_{\mathrm{d}}\left(\frac{\bar{v}}{T_{\mathrm{K}}^{1 / 2}}\right) \bar{\alpha} \frac{n_{\mathrm{tot}} n_{\mathrm{d}}}{n^{2}} .
$$


The value of $C_{\mathrm{gd}}$ is largely determined by the assumptions that we make regarding the chemical state of the gas and the nature of the dust grain population, but in principle it also depends on temperature through the temperature dependence of the mean accommodation coefficient, $\bar{\alpha}$, which quantifies how efficiently energy is shared between dust and gas. There is some debate in the literature concerning the exact functional form of $C_{\mathrm{gd}}$ and its normalization (for example, compare the discussions in Hollenbach and McKee, 1989; Tielens and Hollenbach, 1985; Goldsmith, 2001; Krumholz et al., 2011); however, most values for typical Milky Way conditions are in the range of $C_{\mathrm{gd}}=10^{-33} \mathrm{erg} \mathrm{s}^{-1} \mathrm{~cm}^{3} \mathrm{~K}^{-3 / 2}$ with deviations of about one order of magnitude. The uncertainty in $C_{\mathrm{gd}}$ becomes even greater as we move to lower metallicity, as less is known about the properties of the dust. It is often assumed that $C_{\text {gd }}$ scales linearly with metallicity (e.g. Glover and Clark, 2012c), but this is at best a crude approximation, particularly as the dust abundance appears to scale non-linearly with metallicity in metal-poor galaxies (Galametz et al., 2011; Herrera-Camus et al., 2012).

\subsection{Heating processes}

\subsubsection{Photoelectric heating}

One of the most important forms of radiative heating in the diffuse ISM is photoelectric heating. It is caused by the absorption of UV photons by dust grains which subsequently emit photo-electrons. Their kinetic energy is equal to the difference between the energy of the photon and the energy barrier for detaching the electron from the grain. This difference can be large (of the order of an $\mathrm{eV}$ or more), and the released energy is efficiently redistributed amongst the other gas particles by collisions, causing the gas to heat up.

For standard interstellar dust, following Bakes and Tielens (1994) the photoelectric heating rate per unit volume can be written as

$$
\Gamma_{\mathrm{pe}}=1.3 \times 10^{-24} \epsilon G_{0} n \mathrm{erg} \mathrm{s}^{-1} \mathrm{~cm}^{-3},
$$

where $\epsilon$ is the photoelectric heating efficiency, given by

$$
\epsilon=\frac{0.049}{1+(\psi / 1925)^{0.73}}+\frac{0.037(T / 10000)^{0.7}}{1+(\psi / 5000)},
$$

$G_{0}$ is the strength of the interstellar radiation field in units of the Habing (1968) field, and $T$ is the gas temperature. The parameter

$$
\psi \equiv \frac{G_{0} T^{1 / 2}}{n_{\mathrm{e}}}
$$

is related to the charge of the dust grains in the ISM (Draine and Sutin, 1987; Weingartner and Draine, 2001b). The interpretation is as follows: a high photon flux or a low numbers of of free electrons will lead to dust grains being 
more positively charged, while the converse will lead to grains being more negatively charged. In the latter case electrons can more easily detach and the heating rate is high. The proportionality $T^{1 / 2}$ simply reflects the temperature dependence of the rate coefficient for electron recombination with the grains. For small $\psi, \epsilon \approx 0.05$ when the temperature is low, and $\epsilon \approx 0.09$ when the temperature is high. Since the photons required to eject electrons must be energetic, with minimum energies typically around $6 \mathrm{eV}$, the photoelectric heating rate is highly sensitive to the dust extinction. It becomes ineffective once the visual extinction exceeds values of $A_{V} \sim 1-2$ mag.

\subsubsection{Heating by ultraviolet radiation}

Besides the photoelectric effect, UV photons heat the ISM in two other important ways. First, the photodissociation of $\mathrm{H}_{2}$ results in a transfer of energy to the gas. The absorption process produces a molecule in an excited state, which can break apart and convert the excess energy into kinetic energy through nonradiative decay. As the hydrogen atoms produced in this process have average kinetic energies that are larger than that of the gas particles. The energy release varies depending on which rovibrational level of the excited electronic state was involved in the dissociation (Stephens and Dalgarno, 1973; Abgrall et al., 2000). The average heating rate is around $0.4 \mathrm{eV}$ per dissociation (Black and Dalgarno, 1977). Second, UV irradiation of molecular hydrogen can lead to heating via a process known as UV pumping. The absorption of a UV photon by $\mathrm{H}_{2}$ leads to photodissociation only around $15 \%$ of the time (Draine and Bertoldi, 1996). The rest of the time, the $\mathrm{H}_{2}$ molecule decays back into a bound rovibrational state. Although the molecule sometimes goes back directly into the $v=0$ vibrational ground state, it is far more likely to end up in a vibrationally excited level. In low density gas, it then radiatively decays back to the rovibrational ground state, producing a number of near infrared photons in the process which do not contribute to the overall heating. In high density gas, collisional de-excitation occurs more rapidly, and so most of the excitation energy is converted into heat. In this case, the resulting heating rate is around $2 \mathrm{eV}$ per pumping event, compared to around $10-11 \mathrm{eV}$ per photodissociation (see e.g. Burton et al., 1990). This process becomes significant only above a critical density of $n_{\text {crit }} \sim 10^{4} \mathrm{~cm}^{-3}$. It is thus not a major heat source at typical molecular cloud densities but can become important in dense cores exposed to strong UV radiation fields.

\subsubsection{Cosmic rays}

In the deep interior of molecular clouds, where the gas is well shielded from the interstellar radiation field, both of the above processes become unimportant and the same holds for photoelectric heating. In this case, cosmic rays provide the main source of heat. They can penetrate deeply into molecular clouds and collisionally ionize hydrogen or helium atoms or $\mathrm{H}_{2}$ molecules. The resulting 
ions and electrons typically have very large velocities and so subsequent collisions can trigger secondary ionization events. Eventually the excess kinetic energy gets converted into heat, with the amount of energy transferred depending on the composition of the gas (Dalgarno et al., 1999; Glassgold et al., 2012), but it is typically around 10-20 eV. Most models of thermal balance in dark clouds adopt a heating rate that is a simply fixed multiple of the primary hydrogen cosmic ray ionization rate (see e.g. Goldsmith and Langer, 1978; Goldsmith, 2001; Ceverino et al., 2010; Krumholz et al., 2011). A commonly adopted parameterization is

$$
\Gamma_{\mathrm{cr}} \sim 3.2 \times 10^{-28}\left(\zeta_{\mathrm{H}} / 10^{-17} \mathrm{~s}^{-1}\right) n \mathrm{erg} \mathrm{cm}^{-3} \mathrm{~s}^{-1},
$$

where the cosmic ray ionization rate of atomic hydrogen $\zeta_{\mathrm{H}}$ is scaled by its typical value of $10^{-17} \mathrm{~s}^{-1}$ and $n$ is the number density of hydrogen nuclei. Note that the uncertainty introduced by averaging is typically much smaller than the current uncertainty in the actual cosmic ray ionization rate in the considered region. We note that in most applications the cosmic ray ionization and heating rates are assumed to be constant throughout the cloud. A better approach is

$$
\Gamma_{\mathrm{cr}}=n Q(n) \zeta(n)
$$

with density dependent heating rate $Q(n)$ and cosmic ray flux $\zeta(n)$. Whereas $Q$ only slightly depends on density $n$ (Glassgold et al., 2012), $\zeta$ decreases by orders of magnitude in the densest parts of a cloud (see e.g. Padovani et al., $2009,2018)$. The use of equation (27) leads to more accurate values of $\Gamma_{\text {cr }}$ up to a factor of $\sim 7$.

\subsubsection{X-rays}

X-rays can heat the interstellar medium in a very similar fashion to cosmic rays: X-ray ionization produces an energetic electron that can cause a cascade of secondary ionization events with some fraction of the excess energy going into heat. Unlike cosmic rays, X-rays are sensitive to the effects of absorption, because their mean free path is much smaller. Hence, they can be an important heat source in the diffuse ISM (see e.g. Wolfire et al., 1995), but they are generally thought to be unimportant in the dense gas inside molecular clouds, unless these clouds are located close to a strong X-ray source such as an AGN (see e.g. Hocuk and Spaans, 2010).

\subsubsection{Chemical reactions}

The latent heat associated with chemical reactions can also contribute to the overall thermal energy balance in certain phases of the ISM. For example, the formation of a new chemical bond, such as that between the two hydrogen nuclei in a $\mathrm{H}_{2}$ molecule, releases energy. Much of this energy will be channeled into rotational and/or vibrational modes of the newly-formed molecule. In low density environments, it will rapidly be lost by radiation. At high densities, 


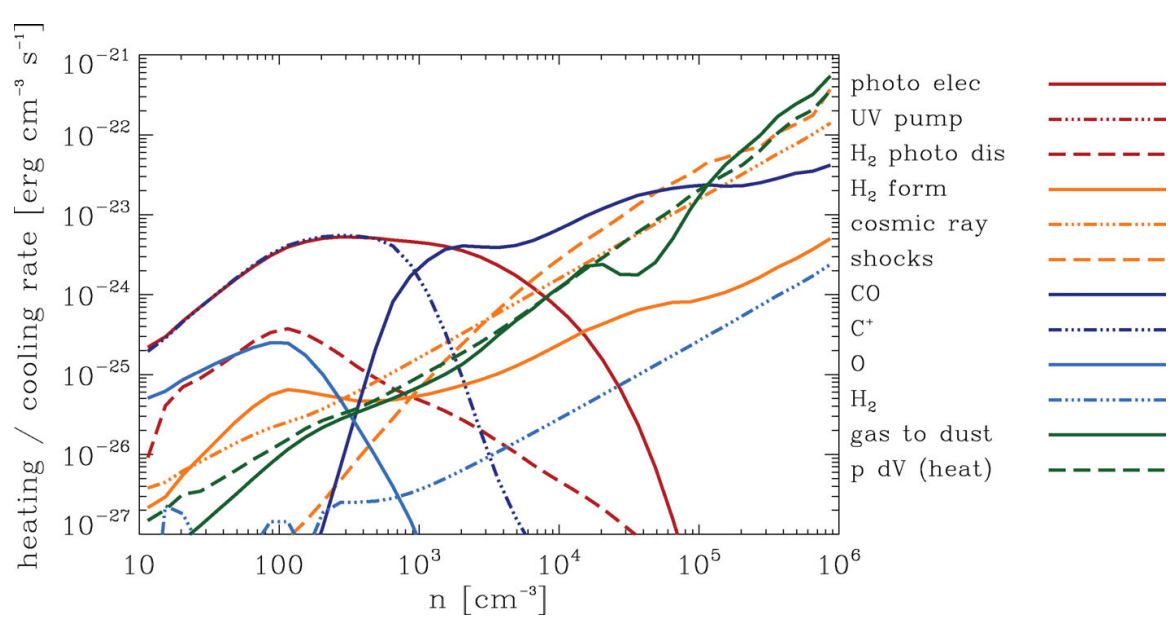

Fig. 1 Illustration of the relative importance of the various heating and cooling processes in the ISM, plotted as a function of the hydrogen nuclei number density, $n$, calculated from a simulation of molecular cloud formation from initially atomic gas in the solar neighborhood. Heating processes are depicted in red and orange, cooling processes in dark and light blue, processes that in principle can either add or remove heat from the gas component of the ISM are shown in green. Figure adapted from Glover and Clark (2012b)

however, collisional de-excitation can convert this energy into heat before it can be lost via radiation.

Some of the energy released in a reaction may also go into motion of the newly-formed molecule, and this will also be rapidly converted into heat via collisions. Many of the reactions occurring in interstellar gas lead to heating in this way, but for the most part, their effects are unimportant. The one case in which this process can become significant is the formation of $\mathrm{H}_{2}$ at high densities. Each event releases a total energy of $4.48 \mathrm{eV}$. With the typical $\mathrm{H}_{2}$ formation rates given by (10) we obtain

$$
\Gamma_{\mathrm{H}_{2} \text { form }} \sim 2 \times 10^{-28} \epsilon_{\mathrm{H}_{2}} n n_{\mathrm{H}} \mathrm{erg} \mathrm{cm}^{-3} \mathrm{~s}^{-1}
$$

for the corresponding heating rate, assuming that the efficiency with which this energy is converted into heat is $\epsilon_{\mathrm{H}_{2}}$. Comparing with the cosmic ray heating rate (26), we see that $\mathrm{H}_{2}$ formation heating will dominate whenever $\epsilon_{\mathrm{H}_{2}} n_{\mathrm{H}}>$ $\left(\zeta_{\mathrm{H}} / 10^{-17} \mathrm{~s}^{-1}\right)$. In principle, $\mathrm{H}_{2}$ formation heating can be important provided that the efficiency factor $\epsilon_{\mathrm{H}_{2}}$ is not too small. Unfortunately, the value of $\epsilon_{\mathrm{H}_{2}}$ is highly uncertain (see e.g. Le Bourlot et al., 2012; Roser et al., 2003; Congiu et al., 2009, and references therein), and more work is required to constrain it.

\subsubsection{Dynamical processes}

Finally, hydrodynamical and magneto-hydrodynamical (MHD) effects can also lead to significant heating. In subsonic, gravitationally collapsing regions, such as low-mass prestellar cores, adiabatic compression ( $P d V$ heating) can be a 
major source of heat and can actually be more important in the overall thermal balance of the core than cosmic ray heating. In less quiescent environments, where the gas flow is supersonic, turbulent dissipation in shocks or regions of strong shear is another major heat source. The same is true for magnetic reconnection and other non-ideal MHD processes in magnetized media (e.g. Momferratos et al., 2014). The rate at which turbulent kinetic energy dissipates in regions of supersonic turbulence is reasonably well established (Mac Low et al., 1998; Stone et al., 1998; Mac Low, 1999). The energy dissipation rate within a cloud of mass $M$ can be written to within a factor of order unity as (Mac Low, 1999)

$$
\dot{E}_{\mathrm{kin}} \sim-M k_{\mathrm{d}} \sigma_{v}^{3},
$$

where $k_{\mathrm{d}}$ is the wavenumber on which energy is injected into the system and where $\sigma_{v}$ is the velocity dispersion at this scale. We assume that it is comparable to the size of the cloud (see e.g. Brunt et al., 2009). Furthermore, we adopt Larson's relations between the size of the cloud and its velocity dispersion, $\sigma_{v} \propto L^{\alpha}$ with $\alpha \sim 0.5$, and number density, $n \propto L^{\beta}$ with $\beta \sim 1$. We note that normalization and slope are both quite uncertain (Larson, 1981; Hennebelle and Falgarone, 2012). Put together, we arrive at an average turbulent heating rate (Pan and Padoan, 2009)

$$
\Gamma_{\text {turb }}=3 \times 10^{-27}\left(\frac{L}{1 \mathrm{pc}}\right)^{0.2} n \mathrm{erg} \mathrm{s}^{-1} \mathrm{~cm}^{-3} .
$$

While dominating on large scales, this heating rate can become comparable to the cosmic ray heating rate on small scales, in more quiescent regions deeply embedded regions of the cloud such as low-mass protostellar cores. We also note that turbulent heating is highly intermittent (Pan and Padoan, 2009). This means that in much of the cloud, the influence of turbulent dissipation is small, while in small, localized regions, very high heating rates can be produced (see e.g. Falgarone et al., 1995; Godard et al., 2009). We provide a more detailed account of properties of turbulence in $\S 4$.

Finally, note that the physical nature of the heating depends upon the strength of the magnetic field within the gas. If the field is weak, energy dissipation occurs mostly through shocks, whereas if the field is strong, a substantial amount of energy is dissipated via non-ideal MHD processes such as ambipolar diffusion, i.e. the drift between the neutral and charged constituents of the ISM (Padoan et al., 2000; Li et al., 2012).

\subsubsection{Heating and cooling in the nearby ISM}

Figure 1 provides an overview of the most important heating and cooling processes for the solar neighborhood ISM. The rates are plotted as a function of the hydrogen nuclei number density, $n$. The figure shows that initially atomic gas exhibits three different regimes. At densities $n<2000 \mathrm{~cm}^{-3}$, the gas heating is dominated by photoelectric emission from dust grains (§2.5.1), while cooling is provided by fine structure emission from $\mathrm{C}^{+}$. In the density 
regime $2000<n<10^{5} \mathrm{~cm}^{-3}$, rotational line emission from CO becomes the main coolant. Photoelectric heating remains the main heat source initially but steadily becomes less effective, owing to the larger visual extinction of the cloud at these densities. Other processes - adiabatic compression of the gas, dissipation of turbulent kinetic energy in shocks and cosmic ray ionization heating - become more important at $n \sim 6000 \mathrm{~cm}^{-3}$ and above. Finally, at densities above about $10^{5} \mathrm{~cm}^{-3}$, the gas couples to the dust (Section 2.4.4), which acts as a thermostat and provides most of the cooling power. Weak shocks and adiabatic compressions together dominate the gas heating in this regime, each contributing close to half of the total heating rate (for a more detailed discussion, see Glover and Clark, 2012b).

\section{Thermal structure of the ISM}

To understand the ISM it is crucial to know its temperature distribution. As mentioned in the previous section, the ISM consists of two atomic phases, namely the cold neutral medium and the warm neutral medium. The latter has a typical density of about $0.5 \mathrm{~cm}^{-3}$ and a temperature of the order of $8000 \mathrm{~K}$. The former is approximately 100 times denser and cooler. We first describe in short the cooling and heating processes that are relevant for the atomic gas, and we then discuss the principle of thermal instability.

\subsection{Thermal balance}

The balance of cooling and heating must be computed to provide the temperature as a function of density. The detailed analysis for the thermal equilibrium state in the neutral atomic phase can be found, for example, in Wolfire et al. (2003). The dominant heating mechanisms are photoelectric emission from small grains and PAHs, ionization by cosmic rays and soft X-rays, and the formation and photodissociation of $\mathrm{H}_{2}$. The FUV field is close to the Habing's value, $G_{0}=1.7$. The most important cooling processes are line emission from $\mathrm{H}, \mathrm{C}, \mathrm{O}, \mathrm{Si}$, and $\mathrm{Fe}$, and rovibrational lines from $\mathrm{H}_{2}$ and $\mathrm{CO}$. Collisions of atomic and molecules with dust grains and the resulting thermal emission from the collisionally heated dust also contribute to cooling.

At high density, typically $10^{3} \mathrm{~cm}^{-3}<n<10^{6} \mathrm{~cm}^{-3}$, the cooling depends on the column density and UV radiation field and molecular cooling dominates. How well the radiation field is shielded, in relative importance of $\mathrm{H}_{2}$ self-shielding and dust shielding is duscussed in Sternberg et al. (2014). The chemistry and cooling for a broad range of density, column density, metallicity, and radiation fields are discussed by Koyama and Inutsuka (2000), Glover and Clark (2012b) and Gong et al. (2017).

Figure 2 displays the phase diagram, i.e. temperature and pressure as a function of density. The interstellar radiation field in this analysis is 1.7 times the Habing field $\left(G_{0}=1.7\right.$, Habing 1968) and is attenuated in regions of high 


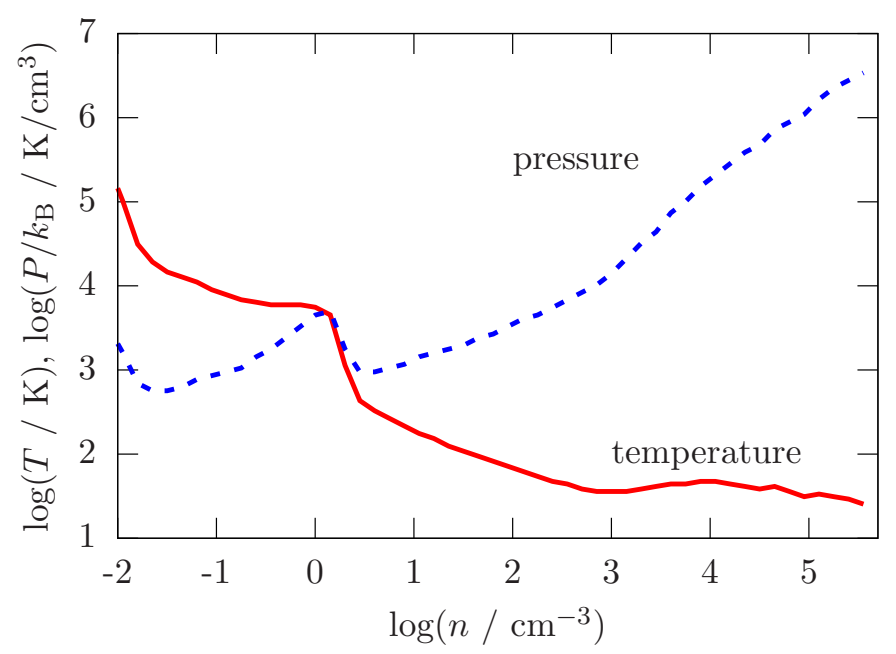

Fig. 2 Phase diagram of the ISM including the most relavant heating and cooling processes Computed using the heating and cooling processes described in Glover and Clark (2012a) implemented in the SILCC ISM simulations (Walch et al., 2015; Girichidis et al., 2016, 2018).

optical depth (Walch et al., 2015; Girichidis et al., 2016). The At high column density, the main cooling process is due to CO molecules up to about $10^{5}$ $\mathrm{cm}^{-3}$, above which dust cooling dominates. Under these conditions, the most important heating mechanism is usually cosmic rays.

\subsection{Thermal Instability}

Thermal instability is believed to play an important role in the ISM. It stems from the fact that cooling is proportional to the square of the gas density (because cooling elements get excited through two-body collisions) while the heating function in some range of temperatures is approximately proportional to the density. Quantitatively the existence of the thermal instability can be related to the slope of heat-loss function, $\mathcal{L}=\rho \Lambda-\Gamma$, where $\rho \Lambda$ is the cooling function per unit volume with the density $\rho$ and the cooling rate $\Lambda$ in units of $\operatorname{erg} \mathrm{cm}^{3} \mathrm{~s}^{-1}$, and $\Gamma$ is the heating function in units of $\operatorname{erg} \mathrm{gs}^{-1}$. The stability conditions of a uniform medium subject to heating and cooling have been studied in great detail by Field (1965), who investigated the impact of isochoric, isentropic and isobaric perturbations on the thermal equilibrium. In the ISM the unstable isobaric perturbation is believed to be the most relevant one and can be expressed as

$$
\left(\frac{\partial \mathcal{L}}{\partial T}\right)_{\mathrm{P}}<0 \Leftrightarrow\left(\frac{\partial P}{\partial \rho}\right)_{\mathcal{L}}<0 .
$$




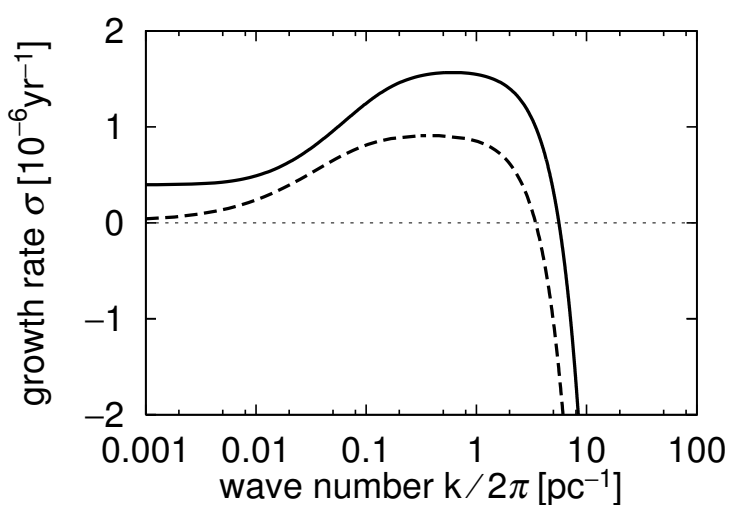

Fig. 3 The dispersion relation for the condensation mode of thermal instability analyzed in Koyama and Inutsuka (2000) for a temperature of $T=10^{3} \mathrm{~K}$ and a pressure of $P / k_{\mathrm{B}} \approx 2000 \mathrm{~K} \mathrm{~cm}^{-3}$. The dashed curve denotes the result for a system at rest in thermal equilibrium. The solid curve denotes the case of an isobarically contracting core (Koyama and Inutsuka, 2000), (CAAS. Reproduced with permission.

The physical meaning of this expression is straightforward. Consider thermal equilibrium, i.e. $\mathcal{L}=0$. If the pressure decreases as the density increases the support against a further increase in density reduces. This thermal state is unstable because any small over-density corresponds to a local pressure minimum and will therefore be amplified. Typically this instability appears for in the temperature regime between $\sim 100$ and $\sim 5000 \mathrm{~K}$. Figure 3 displays the growth rate of thermal instability as a function of wave number. The dashed curve shows the dispersion relation in at thermal equilibrium for a system with uniform density at rest. The solid line indicates the counterpart for an isobarically contracting sphere. Thermal conduction is also taken into account in this calculation, which is why the growth rate vanishes at large wave numbers. As discussed by Field (1965), thermal conduction can counteract thermal instability because it smooths out small scale temperature perturbations. The critical wavelength, i.e. the one at which the growth rate becomes zero, is now called the "Field length" and is given by

$$
\lambda_{\mathrm{F}} \equiv \sqrt{\frac{K T}{\rho^{2} \Lambda}} .
$$

Here $K$ denotes the coefficient of thermal conduction. The peak of the growth rate occurs at a wavelength that is several times larger than the Field length. Therefore, thermal instability leads to structures larger than the Field length.

The effect of magnetic fields on the linear growth of thermal instability was studied in detail by Ames (1973). A sufficiently strong magnetic field suppresses motions perpendicular to the field lines assuming flux freezing. Consider for instance a slab geometry, in which the magnetic pressure is simply proportional to the density squared (since the magnetic field is proportional to the density) and therefore an increase in the magnetic pressure can compensate 
for a decrease in thermal pressure. However, perturbations in the direction along the magnetic field are not suppressed and remain unstable if the cooling function satisfies the instability criteria.

\subsection{Thermal front propagation and pressure regulation}

The non-linear development of thermal instability has been studied by various authors (e.g. Hennebelle and Pérault, 1999; Koyama and Inutsuka, 2000; Piontek and Ostriker, 2004; Inoue et al., 2007; van Loo et al., 2007; Choi and Stone, 2012), see also Elphick et al. (1992) and Shaviv and Regev (1994) for the dynamics of fronts in thermally bistable media. Typically, after the linear phase, a non-linear structure develops and eventually a cloud of dense gas embedded in the warm surrounding medium settles into an equilibrium. The dense cloud is then connected to the WNM through thermal fronts, whose thicknesses are given by the Field length (Field, 1965; Begelman and McKee, 1990; Stone and Zweibel, 2010; Kim and Kim, 2013). Note that the Field length varies from about $10^{-3} \mathrm{pc}$ in the CNM to $0.1 \mathrm{pc}$ in the WNM and the fronts have a thickness that can be approximated by the geometric mean of the Field length in the cold and warm medium (e.g. Kim and Kim, 2013). The fronts themselves are not in thermal equilibrium, since the denser part is cooling while the more diffuse one is heating. Thus, except for a particular value of the pressure for which the two contributions are equal (also called saturation pressure), it is generally the case that either the cloud evaporates or condenses. If the ambient pressure is lower than the saturation pressure the thermal front becomes an evaporation front. Conversely, if the ambient pressure is larger than the saturation pressure the front leads to a condensation (e.g. Inoue et al., 2006).

\section{Turbulence}

\subsection{Introduction}

Fluid flows are often divided into two sharply different categories: quiet smooth flows known as laminar, and turbulent flows in which the fluid velocity exhibits chaotic fluctuations in both space and time over a wide range of length and time scales. The turbulent velocity field is unpredictable because small variations in the initial conditions produce large changes to the subsequent motion. This irregular state of motion is a truly remarkable feature of the governing Navier-Stokes (NS) equation, describing the rate of change of velocity in a viscous fluid,

$$
\partial_{t} \boldsymbol{u}+(\boldsymbol{u} \cdot \boldsymbol{\nabla}) \boldsymbol{u}=-\nabla p / \rho+\nu \nabla^{2} \boldsymbol{u}+\boldsymbol{f} / \rho,
$$

under suitable initial and boundary conditions. This equation is simply Newton's law for a fluid, but it includes a seemingly innocent nonlinear inertial term, $(\boldsymbol{u} \cdot \boldsymbol{\nabla}) \boldsymbol{u}$, where the fluid velocity, $\boldsymbol{u}(\boldsymbol{x}, t)$, appears in a quadratic form. 
It is this nonlinearity in the deterministic NS equation that brings about a source of chaos with it. The velocity must further satisfy a simplified form of the continuity equation $\boldsymbol{\nabla} \cdot \boldsymbol{u}=0$, if the density of the fluid is constant, $\rho=\rho_{0}$. Finally, $\nu$ is the kinematic viscosity, and $\boldsymbol{f}(\boldsymbol{x}, t)$ is a random external force, which is usually referred to as driving.

The emergence of turbulence only occurs at sufficiently large Reynolds numbers, $R e=U L / \nu$, which measures the ratio of the inertial term to the viscous term in Eq. (33), assuming $U$ is the characteristic (e.g. root mean squared) velocity of energy-containing eddies ${ }^{1}$ of size, $L$.

Turbulent fluids are not, however, completely random and unpredictable. Instead their statistics are reproducible and strictly obey certain scaling laws, which can be derived from the NS equation under simplifying assumptions about the symmetries of the underlying problem.

\subsection{Basic probabilistic tools}

The scale-dependent correlations of fluid variables in turbulent flows are traditionally described in terms of structure functions, correlation functions, and their Fourier counterparts (e.g. power spectra). These standard probabilistic tools are widely used in studies of incompressible turbulence in which the velocity field $\boldsymbol{u}(\boldsymbol{x}, t)$ fully describes the system. The velocity of a turbulent fluid at a given point in space and in time can be treated as a vector-valued centered random variable (zero mean value $\langle\boldsymbol{u}\rangle=0$ ), as it would still be a function of the initial conditions.

Let us consider the velocity increment, $\delta \boldsymbol{u}(\boldsymbol{x}, \boldsymbol{r})=\boldsymbol{u}(\boldsymbol{x}+\boldsymbol{r})-\boldsymbol{u}(\boldsymbol{x})$, between two points, $\boldsymbol{x}$ and $\boldsymbol{x}+\boldsymbol{r}$, separated by the lag, $\boldsymbol{r}$. In homogeneous systems, the statistics of $\delta \boldsymbol{u}(\boldsymbol{x}, \boldsymbol{r})$ only depends on the lag, $\boldsymbol{r}$, and not on the position, $\boldsymbol{x}$. If turbulence is also isotropic, the statistics of velocity increments would depend only on the magnitude of the lag, $r=|\boldsymbol{r}|$, not its orientation. Homogeneity and isotropy imply that spatial translations, rotations, and reflections of the original system of coordinate axes $\left(x_{1}, x_{2}, x_{3}\right)$ do not change the distribution functions of physical variables. ${ }^{2}$ It is convenient then to define the longitudinal velocity increment as $\delta u_{\|}(r)=\delta \boldsymbol{u}(\boldsymbol{r}) \cdot \boldsymbol{r} / r$, using the natural coordinate frame with the direction of one of the coordinate axes aligned with $\boldsymbol{r}$. The two remaining transverse components, $\delta u_{\perp}(r)$, are equal in the isotropic case. The longitudinal and transverse velocity structure functions of order $p$ are defined, respectively, as $S_{p}^{\|}(r)=\left\langle\left[\delta u_{\|}(r)\right]^{p}\right\rangle$ and $S_{p}^{\perp}(r)=\left\langle\left[\delta u_{\perp}(r)\right]^{p}\right\rangle$, where $\langle\cdot\rangle$ denotes

\footnotetext{
1 In fluid dynamics, an eddy is understood as the swirling current of a fluid or a 'blob of vorticity', but there is a good tradition in hydrodynamic turbulence of avoiding any formal definition of a turbulent eddy, see page 52 in Davidson (2004).

2 In realistic situations, both assumptions are usually not satisfied at large scales. However, if they are valid at small scales and far from boundaries of the flow or its other special regions, this general theoretical framework still remains useful.
} 
averaging over an ensemble of point pairs ${ }^{3}$ with the fixed lag magnitude $r$. Note that the velocity structure functions retain Galilean invariance because they depend on $\delta \boldsymbol{u}$.

The two-point velocity autocorrelation function is defined as $R_{\boldsymbol{u} u}(\boldsymbol{r})=$ $\langle\boldsymbol{u}(\boldsymbol{x}) \cdot \boldsymbol{u}(\boldsymbol{x}+\boldsymbol{r})\rangle$, also assuming homogeneity. One can readily show that the velocity autocorrelation function is related to the second order structure function as $R_{\boldsymbol{u} \boldsymbol{u}}(\boldsymbol{r})=\left\langle\boldsymbol{u}^{2}\right\rangle-\left\langle[\delta \boldsymbol{u}(r)]^{2}\right\rangle / 2$ and also that $R_{\boldsymbol{u} \boldsymbol{u}}(\mathbf{0})=\left\langle\boldsymbol{u}^{2}\right\rangle$. In addition, the three-dimensional power spectrum of velocity, $P_{3 D}(\boldsymbol{u}, \boldsymbol{k}) \equiv|\widehat{\boldsymbol{u}}(\boldsymbol{k})|^{2}=\widehat{R_{\boldsymbol{u} \boldsymbol{u}}}(\boldsymbol{k})$, is the Fourier transform of the autocorrelation function, as follows from the Wiener-Khinchin formula (Wiener, 1930; Khintchine, 1934) (here ... denotes the Fourier transform ${ }^{4}$ and $\boldsymbol{k}$ is the wave vector). If the turbulence is also isotropic then the 3D power spectrum depends only on the magnitude of the wave vector, $k=|\boldsymbol{k}|$, and hence the one-dimensional (angle-integrated) spectrum can be written as $P(\boldsymbol{u}, k) \equiv \int P_{3 D}(\boldsymbol{u}, \boldsymbol{k}) d \Omega_{\boldsymbol{k}}=4 \pi k^{2} P_{3 D}(\boldsymbol{u}, k)$. From Parseval's theorem we further get $\int_{0}^{\infty} P(\boldsymbol{u}, k) d k=\int P_{3 D}(\boldsymbol{u}, \boldsymbol{k}) d \boldsymbol{k}=\left\langle\boldsymbol{u}^{2}\right\rangle=$ $R_{\boldsymbol{u}, \boldsymbol{u}}(0)$, which relates various second order moments introduced above to the mean turbulent kinetic energy, $E=\rho_{0}\left\langle\boldsymbol{u}^{2}\right\rangle / 2$, in the incompressible case. Another useful quantity, the velocity dispersion $\sigma_{u}(r)$ as a function of scale $r=2 \pi / \kappa$, can be easily computed from the power spectrum since the variance $\sigma_{u}^{2}(r)=\int_{\kappa}^{\infty} P(\boldsymbol{u}, k) d k$, if $\langle\boldsymbol{u}\rangle=0$, and interpreted as a result of high-pass filtering operation.

\subsection{Energy cascade and the four-fifths law}

In the classical phenomenology of turbulence, kinetic energy is supplied at the largest scales (e.g. by a stochastic forcing mechanism). Non-linear advection coupled with fluid instabilities then generates motions on progressively smaller and smaller scales. This energy transfer process continues until molecular transport becomes dominant and dissipates the energy as heat. In such scale-by-scale energy cascade ${ }^{5}$ powered by vortex stretching in three dimensions, fluid motions progressively lose information about (non-universal) details of large-scale energy injection, leading to presumably self-similar (universal) fluid behavior at small scales. This self-similarity leads to a power-law energy spectrum determined solely by the magnitude of the energy flux if we (heuristically) assume that the cascade interactions are local, i.e. only comparable spatial scales interact with one another. The locality assumption does

\footnotetext{
3 Relevant methods of taking averages of random functions of position and time in homogeneous and ergodic systems are discussed, for instance, in section 2.1 of Batchelor (1953) and in section 4.4 of Frisch (1995).

4 Note that the Fourier transforms of homogeneous random functions are, generally, random distributions, i.e. not ordinary functions of their argument, $\boldsymbol{k}$. One way to deal with this mathematical difficulty is to replace the ordinary integrals with generalized stochastic Fourier-Stieltjes integrals (Batchelor, 1953); another way is to use low- or high-pass filtering (e.g. coarse-graining), which allows one to deal lusively with ordinary functions (Frisch, 1995).

5 Envisioned by Lewis F. Richardson in 1922 (Richardson, 1965).
} 
not hold universally true, though, as there are non-local scale interactions in Fourier space (see, e.g., section 7.3 in Frisch (1995) and section 5.5 in Diamond et al. (2010)).

To describe the scale-by-scale energy balance, one can write the NS equation in Fourier space and use Fourier integrals to describe turbulent flow in a bounded region of an infinite space, $\mathbb{R}^{3}$. The resulting equation takes the form (e.g. Frisch, 1995) $\partial_{t} E(k)=T(k)-D(k)+F(k)$, where $\partial_{t}$ denotes the partial time derivative, $T(k)$ is the transfer function describing energy transfer to scale $k$ due to nonlinear interaction of velocity fluctuations at all different scales, $D(k)=2 \nu k^{2} E(k)$ describes the viscous dissipation of kinetic energy into heat, and $F(k)$ accounts for the energy supply to the system due to the work of an external force. If turbulence is statistically stationary, $E(k)$ does not change with time at all wave numbers, $k$, and hence $T(k)-D(k)+F(k)=0$. In this case, energy injection and dissipation rates are balanced overall. Note that energy supply is mostly concentrated at large scales (small $k$ ), while energy dissipation occurs at small scales (large $k$ ), see Fig. 4 . Balance between energy injection and dissipation implies that the areas under $F(k)$ and $D(k)$ curves are equal. Since in Fig. 4 the energy is supplied on the left and removed on the right, it should be transferred across the range $k \in\left[k_{f}, k_{\eta}\right]$ where $F(k)=D(k)=0$ and hence $T(k)=0$, i.e. all energy incoming to $k$ from larger scales gets transferred to smaller scales. The range of scales that are sufficiently distant from both $k_{f}$ and $k_{\eta}$, where we expect self-similar behavior of fluctuations, is called the inertial range. We can also define the kinetic energy contained at all scales smaller than a given scale as $\int_{k}^{\infty} E(\kappa) d \kappa$ and the cross-scale energy flux as $\Pi(k)=\int_{k}^{\infty} T(\kappa) d \kappa$; similarly, $\Phi(k)=\int_{k}^{\infty} F(\kappa) d \kappa$ and $\Delta(k)=\int_{k}^{\infty} D(\kappa) d \kappa$. In statistically stationary turbulence, these quantities are related: $\Pi(k)-\Delta(k)+\Phi(k)=0$. In the inertial range (shown in green in Fig. 4), the energy transfer rate is independent of scale, $\Pi(k)=$ const. It is also equal to the energy dissipation rate, $\epsilon$, and to the energy injection rate, $\langle\boldsymbol{u} \cdot \boldsymbol{f}\rangle$, associated with the driving force: $\Pi(k)=\Delta(0) \equiv \epsilon=\Phi(0)=\langle\boldsymbol{u} \cdot \boldsymbol{f}\rangle$.

Suppose that eddies of size $r$ participating in this energy transfer process in the inertial range have a characteristic velocity, $u_{r}$, and break up on the eddy turn-over time, $\tau_{r} \equiv r / u_{r}$. If the energy flux, $\Pi(r)$, along the cascade is constant (we consider a statistically stationary situation) and equal to the energy dissipation rate, $\epsilon$, then $\Pi(r) \sim u_{r}^{2} / \tau_{r} \sim u_{r}^{3} / r \sim \epsilon$. In terms of the first-order velocity structure function, this can be written approximately as $S_{1}(r)=C_{1}(\epsilon r)^{1 / 3}$, where $C_{1}$ is a dimensionless constant, which is usually called the Kolmogorov-Obukhov law Kolmogorov (1941a); Obukhov (1941). It was pointed out by Onsager (1949) that the $r^{1 / 3}$ law for velocity increments may reflect the lack of smoothness of the velocity field, which is only Hölder continuous of exponent $1 / 3$ (see, e.g., section 1.3 in Feireisl et al. (2016) for the definition of Hölder continuous functions). Onsager was perhaps the first to raise the issue of singularities in turbulent fluid flows (Frisch, 1995; Eyink and Sreenivasan, 2006). Using dimensional arguments, the Kolmogorov-Obukhov law can be extended to structure functions of ar- 


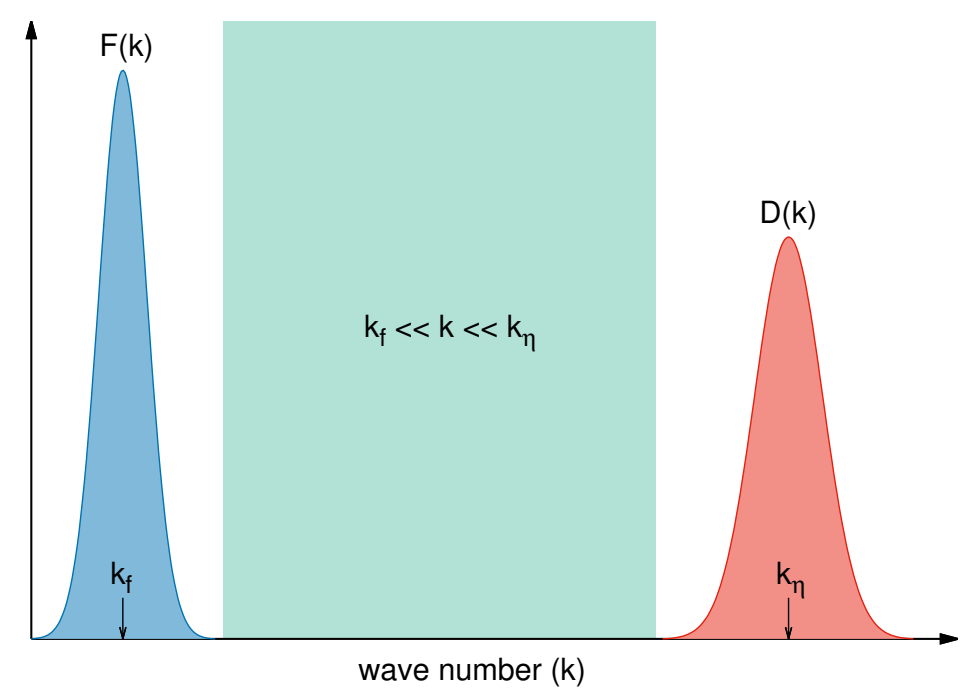

Fig. 4 Sketch of scale-by-scale energy budget in spectral space. $F(k)$ indicates the forcing range centered around $k_{f}, D(k)$ shows the dissipation range around $k_{\eta}=2 \pi / \eta$; the inertial range of scales in between, $k_{f} \ll k \ll k_{\eta}$, is shown in green.

bitrary order $p$ as $S_{p}(r)=C_{p}(\epsilon r)^{p / 3}$. Similarly, the energy spectrum can be written as $E(k)=2 \pi k^{2}|\widehat{\boldsymbol{u}}(\boldsymbol{k})|^{2}=C_{K} \epsilon^{2 / 3} k^{-5 / 3}$, where $C_{K}$ is the Kolmogorov constant. Since the dissipation scale, $\eta$ (also called the Kolmogorov scale), depends only on the dissipation rate, $\epsilon$, and on the viscosity, $\nu$, the Kolmogorov scale can be readily written as $\eta=\left(\nu^{3} / \epsilon\right)^{1 / 4}$ using dimensional arguments (see Eq. (6.66) in Frisch, 1995).

It is important to emphasize that most of the scaling relations introduced above are approximations since they were obtained phenomenologically. The only exception is the exact expression for the third order longitudinal velocity structure function, $S_{3}^{\|}(r)=-\frac{4}{5} \epsilon r$, derived by Kolmogorov from the NS equations Kolmogorov (1941b). This result, known as the four-fifths law, is valid for homogeneous isotropic stationary turbulence in the inertial range. A primitive form of the four-fifths law for isotropic turbulence, which served as a stepping stone for the derivation, combines longitudinal and vector velocity increments, $\left\langle\delta u_{\|}(r)|\delta \boldsymbol{u}(r)|^{2}\right\rangle=-\frac{4}{3} \epsilon r$, and is known as the four-thirds law or the von Kármán-Howarth relation (de Karman and Howarth, 1938). ${ }^{6}$

The third-order moment of velocity increments is special, since it depends on the mean dissipation rate, $\epsilon=\langle\varepsilon\rangle$. Note that the local dissipation rate, $\varepsilon$, is not a constant but rather a random variable with its own probability

6 A similar relation, $\left\langle\delta u_{\|}(r)[\delta \theta(r)]^{2}\right\rangle=-\frac{4}{3} \epsilon_{\theta} r$, was obtained by Yaglom (1949) for temperature fluctuations in turbulent flows. Here, $\delta \theta(r)$ is the temperature increment and $\epsilon_{\theta}$ is the mean dissipation rate of temperature fluctuations. An anisotropic generalization of this relation exploited in Galtier and Banerjee $(2011), \boldsymbol{\nabla} \cdot\left\langle|\delta \boldsymbol{u}(\boldsymbol{r})|^{2} \delta \boldsymbol{u}(\boldsymbol{r})\right\rangle=-4 \epsilon$, avoids projection onto the $\|$ direction and is sometimes called the von Kármán-Howarth-Monin relation (Frisch, 1995; Antonia et al., 1997). 
density function (PDF). Moments of order $p \neq 3$ depend on $\left\langle\varepsilon^{p / 3}\right\rangle \neq \epsilon^{p / 3}$, and hence their scaling relations cannot be universal functions of $\epsilon$ and $\nu$ as they depend on the detailed structure of the $\varepsilon-\mathrm{PDF}$, which may be different in different turbulent flow realizations. This lack of universality was first noticed by Landau in his famous remark made at a seminar in Kazan in early 1942, where Kolmogorov has summarized his 1941 work (Landau and Lifshitz, 1987; Frisch, 1995).

As noted by Uriel Frisch (Frisch, 1995, Section 6.2), the four-fifths law "is one of the most important results in fully developed turbulence, because it is both exact and non-trivial. It thus constitutes a kind of 'boundary condition' on theories of turbulence: such theories, to be acceptable, must either satisfy the four-fifths law or explicitly violate the assumptions made in deriving it."

\subsection{Effects of compressibility}

Turbulence in the ISM, like in many other astrophysical environments (e.g. solar wind and stellar winds, supernova collapse, black hole accretion flows) and in numerous terrestrial applications (e.g. supersonic mixing in scramjets, hypersonic turbulent boundary layers in high speed aerodynamics, shockturbulence interaction, inertial confinement fusion, volcanic eruptions, etc.) is highly compressible and hence the formalism developed for incompressible fluids does not directly apply.

In incompressible turbulence, the pressure is not an autonomous thermodynamic variable, but acts as an enslaved Lagrange multiplier connected to the solenoidal constraint on the velocity. Hence the full solution is contained in the solenoidal (divergence-free) velocity field, see Section 2.1.3 in Sagaut and Cambon (2018).

The next level of complexity is represented by a family of compressible barotropic models where the pressure is a function of density only, $p=p(\rho)$. The specific internal energy (or rather the specific Gibbs free energy) in barotropic flows is interpreted as $e \equiv P(\rho)$, where the pressure potential $P(\rho) \equiv-\int_{\rho_{0}}^{\rho} p(\rho) d(1 / \rho)$. The continuity and NS equations in this case form a closed system and the changes to internal energy in such barotropic flows are interpreted in a purely mechanical sense as resulting from the work done on a volume element of a fluid. Two simple examples include an isothermal closure, $p(\rho)=c_{\mathrm{s}}^{2} \rho$, where the sound speed $c_{\mathrm{s}}=$ const, and a family of polytropic models, $p(\rho)=a \rho^{\gamma}$, where $\gamma>1$ is the adiabatic constant and $a=$ const $>0$ (Batchelor, 1967; Feireisl, 2004). In both cases, compressibility brings along a new scalar field, $\rho$, and a new vector field of dilatational (irrotational) velocity, $\boldsymbol{u}_{\mathrm{d}}$. This compressible velocity component is responsible for pressure-dilatation effects and for the new channel of dilatational dissipation. Note that, while ideal barotropic models provide a mathematically more tractable description for molecular fluids, inclusion of molecular transport effects in the momentum equation is problematic (e.g. Chandrasekhar (1951); Eyink and Drivas (2018), see also Batchelor (1967)). 
In non-barotropic fluids, strong coupling of momentum with thermodynamics becomes important due to pressure-dilatation work (Aluie, 2013) and baropycnal work (Lees and Aluie, 2019). This brings into play fundamentally different energy transfer pathways, missing in the incompressible turbulence (Sagaut and Cambon, 2018). Moreover, various constitutive equations may bring new physical nonlinearities in addition to the usual geometric nonlinearity associated with the Eulerian description (Feireisl, 2004).

To better understand the differences between turbulent fluctuations in incompressible and compressible fluids, it is instructive to consider linearized fluctuation modes on a uniform background. Linear decomposition (Kovásznay, 1953 ) yields three modes of fluctuations: (i) the vortical mode is purely incompressible, includes solenoidal velocity, $\boldsymbol{u}_{\mathrm{sv}}\left(\boldsymbol{\nabla} \cdot \boldsymbol{u}_{\mathrm{sv}}=0\right)$, and no pressure or density fluctuations; (ii) the acoustic mode, which includes only dilatational velocity, $\boldsymbol{u}_{\mathrm{da}}\left(\boldsymbol{\nabla} \times \boldsymbol{u}_{\mathrm{da}}=0\right)$, and isentropic pressure and density fluctuations; and (iii) the entropy mode, which is a wave-like linear solution with entropy fluctuations, no pressure fluctuations, and purely dilatational velocity, $\boldsymbol{u}_{\mathrm{de}}$. The motions in the entropy mode are induced by the viscous effects, hence in the inviscid case $\boldsymbol{u}_{\mathrm{de}}=0$.

In the context of Helmholtz (1858) decomposition for the velocity field, $\boldsymbol{u}=\boldsymbol{u}_{\mathrm{s}}+\boldsymbol{u}_{\mathrm{d}}$, one obtains $\boldsymbol{u}_{\mathrm{s}}=\boldsymbol{u}_{\mathrm{sv}}$ and $\boldsymbol{u}_{\mathrm{d}}=\boldsymbol{u}_{\mathrm{da}}+\boldsymbol{u}_{\mathrm{de}}$ for the viscous case. As can be seen, the solenoidal velocity component does not include the acoustic waves, but the dilatational field is not limited to acoustic phenomena and generally includes convective effects of heat transfer. It is worth noting that the purely kinematic Helmholtz decomposition is exact and does not rely on any small parameter expansion, see Chapters 2 and 3 in Sagaut and Cambon (2018) for an in-depth discussion of various decomposition techniques for compressible flows.

The linear modes of a uniform state we discussed above are decoupled in the first order in amplitude but become fully coupled already in the second order due to mean gradients and nonlinear inertia terms in the dynamical equations (Moyal, 1952; Chu and Kovasznay, 1957). It should be emphasized that such modal decomposition would fail as a general approach to deal with genuinely nonlinear dynamics of turbulence, since corresponding solutions of NS equations cannot be consistently expanded in linear modes. Even though for second-order moments at relatively low turbulent Mach numbers $\left(M \equiv \sqrt{\left\langle u^{2}\right\rangle} /\left\langle c_{\mathrm{s}}\right\rangle=0.1-0.6\right)$ the predictions of the linear approximation are accurate at a few percent level, the errors inevitably become large for higher-order moments and as fluctuations get stronger at higher Mach numbers (Donzis and Jagannathan, 2013; Eyink and Drivas, 2018).

The solenoidal and dilatational Helmholtz projections of the velocity vector $\boldsymbol{u}$ are locally orthogonal in Fourier space (since $\boldsymbol{k} \cdot \widehat{\boldsymbol{u}_{\mathrm{s}}}(\boldsymbol{k})=0$ and $\boldsymbol{k} \times \widehat{\boldsymbol{u}_{\mathrm{d}}}(\boldsymbol{k})=0$ ) and hence the variance $\langle\boldsymbol{u} \cdot \boldsymbol{u}\rangle$ can be split into a sum of the dilatational and solenoidal parts, regardless of the strength of the fluctuations. ${ }^{7}$ A useful diag-

\footnotetext{
7 The same splitting based on $\boldsymbol{u}_{\mathrm{s}}$ and $\boldsymbol{u}_{\mathrm{d}}$ does not work for the Reynolds stress $R_{i j} \equiv$ $\left\langle\rho u_{i} u_{j}\right\rangle$, since in addition to solenoidal and dilatational stresses, there is also non-zero cross Reynolds stress (Lele, 1994).
} 
nostic of compressibility levels in the energy containing range of fully developed homogeneous turbulence is the ratio, $\chi \equiv\left\langle\boldsymbol{u}_{\mathrm{d}} \cdot \boldsymbol{u}_{\mathrm{d}}\right\rangle /\left\langle\boldsymbol{u}_{\mathrm{s}} \cdot \boldsymbol{u}_{\mathrm{s}}\right\rangle$. Together with $R e$ and $M$, the compressive ratio, $\chi$, determines the scaling Donzis and Panickacheril (2019). At low Mach numbers $(M<1)$ the solenoidal and dilatational modes are mostly decoupled and the statistics of turbulence depend either on $\chi_{\mathrm{i}}$, corresponding to initial conditions (in decaying turbulence), or on $\chi_{f}$, reflecting the mode mixture in the forcing (in stationary turbulence). The lack of mode coupling allows drastically different turbulence regimes with vortical and acoustic modes mixed in different proportions controlled by external factors.

The idea of universal scaling classes at low Mach numbers was explored in (Donzis and Panickacheril, 2019) with a diverse set of direct numerical simulations (DNS) (including homogeneous isotropic turbulence and homogeneous shear turbulence). In DNS of homogeneous turbulence with purely solenoidal forcing at $M \geq 0.4$, the dilatational part of the specific kinetic energy is dominated by the acoustic component. If $M<0.4$, small scales are dominated by the pseudo-sound component ${ }^{8}$ associated with hydrodynamic eddies (Wang et al., 2017c). In all cases with solenoidal forcing, the fraction of specific kinetic energy in dilatational modes is small, $\chi<0.1$. When dilatational forcing is used at $M<0.25$, the compressive ratio $\chi$ can be as high as $\approx 2.5$. At $M \sim 0.6$, the compressive ratio, $\chi$, still can be as high as $\sim 1$, but the nonlinear mode coupling would limit its further growth (Donzis and Panickacheril, 2019).

The energy exchange between vortical and acoustic modes further leads to equipartition at $M>1$, as predicted by Kraichnan (1955) for the inviscid case with weak excitation based on Liouville's theorem. Since the acoustic energy includes two equal parts (kinetic + potential) and these are in detailed equilibrium across scales (Sarkar et al., 1991; Donzis and Jagannathan, 2013), the dilatational kinetic energy associated with acoustic waves represents only one half of the full acoustic energy. Thus the expected asymptotic equilibrium value, reflecting energy equipartition between hydrodynamic (eddies) and acoustic (waves) parts of supersonic turbulence, is $\chi_{\infty}=1 / 2$, which corresponds to the so-called 'natural' mix of the modes reproduced in simulations of supersonic turbulence (Kritsuk et al., 2010).

A somewhat lower value of the compressive ratio, $\chi \approx 0.3$ was measured in a simulation of supernova driven magnetized molecular cloud turbulence (Pan et al., 2016). The energy injection mechanism in this simulation can be described as stochastic thermal forcing, which is different from the standard stochastic large-scale acceleration approach. ${ }^{9}$ The resulting compressive ratio,

\footnotetext{
8 There are hydrodynamic and acoustic pressure fluctuations. The pseudo-sound represents vortical pressure fluctuations advected with the fluid velocity, while acoustic waves propagate at the speed of sound. In the pseudo-sound component, the dilatational velocity field is in equilibrium with the solenoidal pressure. Both types of fluctuations can be measured by the observer.

9 Thermal forcing can also be facilitated through a generalized cooling function (a volumetric source in the energy conservation law (Kritsuk and Norman, 2002)) or through a large-scale injection of internal energy (Wang et al., 2019).
} 
nevertheless, is comparable to the value found in isothermal simulations with random solenoidal forcing at $M \approx 9$ and Alfvénic Mach numbers from 3 to 5 (Kritsuk et al., 2010) and deviates from $1 / 2$, as expected, due to different equipartition constraints in magnetohydrodynamic (MHD) turbulence.

Thus, in fully developed compressible turbulence, the Mach number and the compressive ratio, $\chi$, define the scaling universality classes in subsonic regimes, while in supersonic turbulence the compressive ratio assumes an asymptotic value, $\chi_{\infty}$, which only depends on the nature of the system (e.g. the magnetization level) and does not have to depend on details of the energy injection mechanism.

If this phenomenology is correct, in an idealized case of homogeneous supersonic turbulence in a periodic box with large-scale forcing, the energy cascade proceeds from the injection scale with dilatational and solenoidal modes tightly coupled until the sonic scale. ${ }^{10}$ At the sonic scale, the compressive ratio still remains close to its asymptotic 'natural' value; at scales below the sonic scale the hydrodynamic and acoustic cascades fully decouples and proceeds independently without active energy exchange.

Observational measurements of $\chi$ in molecular clouds are difficult, primarily due to projection effects. Therefore, methods developed for nearby molecular clouds for which high-sensitivity, high spatial dynamic range spectral line observations are available, have to rely on the assumption of statistical isotropy and hence cannot account for anisotropies caused by the magnetic fields or large-scale energy injection (Brunt et al., 2010). Another limiting factor is the emission-weighted nature of observational data, which implies the use of Helmholtz decomposition of the momentum density $\boldsymbol{j} \equiv \rho \boldsymbol{u}$ instead of the velocity $\boldsymbol{u}$ (Brunt and Federrath, 2014). It is remarkable that the global compressive ratio measured this way for the Orion B molecular cloud with a mean $\mathcal{M} \sim 6$, using ${ }^{13} \mathrm{CO}(J=1-0)$ data in the $11 \times 6 \mathrm{pc}^{2}$ area encompassing the cloud, yields $\chi \lesssim 0.4$ (Orkisz et al., 2017) - in reasonable agreement with the prediction based on the energy equipartition conjecture discussed above.

4.5 Scaling relations and energy cascades in compressible turbulence

\subsubsection{Numerical simulations}

Most of what we know about turbulence dynamics in the local interstellar medium comes from computer simulations. In a very abstract way such simulations provide us with at least a 12-dimensional data hyperspace (position, time, density, pressure, velocity and magnetic field components), while observations usually yield severely reduced volumes of information, resulting from line-of-sight projection effects and intricate convolutions (e.g. two-dimensional emission maps or 3D position-position-velocity cubes). Because of this dramatic information loss, the only sensible approach to test models is through

\footnotetext{
10 The sonic scale, $k_{\mathrm{S}}$, is defined as a scale at which the root mean squared velocity fluctuations are equal to the mean sound speed, $\int_{k_{\mathrm{s}}}^{\infty} E(k) d k=\left\langle c_{\mathrm{s}}\right\rangle^{2}$.
} 
systematic synthetic observations that mimic the involved convolutions in a realistic way (e.g. Orkisz et al. (2017)).

Interstellar turbulence regimes of interest for star formation studies involve sonic rms Mach numbers from $\mathcal{M} \lesssim 1$ through $\sim 15$ and Alfvénic Mach numbers from $\sim 0.5$ through $\sim 5$ (Kritsuk et al., 2017). These conditions cannot be reproduced in the laboratory, with perhaps a few exceptions (e.g. White et al., 2019). Hence to obtain insights into the physics of interstellar turbulence one has to pursue theoretical research and numerical experiments. One has to keep in mind that realism of star formation simulations is limited by the power of available computational resources and can be improved by advances in numerical methods.

Over the past decade, well-resolved DNS of subsonic regimes with a primary focus on engineering applications have become substantially more mature, as they now routinely use high-order accurate and computationally efficient numerical methods (Wang et al., 2010a), grids up to $2048^{3}$ and Taylor microscale Reynolds numbers up to $R_{\lambda}=430$. Emphasis is being placed on creating a database with various simulation cubes populating the vast parameter space (e.g. Donzis and Jagannathan, 2013; Chen et al., 2015; Jagannathan and Donzis, 2016; Donzis and Panickacheril, 2019; Wang et al., 2017c,b,a).

For comparison, simulations of homogeneous isotropic incompressible turbulence in a periodic box with pseudo-spectral methods aimed at improving our understanding of turbulence small-scale structure have reached a resolution of $8192^{3}$ and $R_{\lambda}=1300$ in 2015 (Yeung et al., 2015). The next steps involve a new pseudo-spectral code that scales up to a problem size of $18,432^{3}$ on the GPU-based supercomputer Summit at ORNL and will continue to focus on intermittency in incompressible turbulence (Yeung, 2019).

The biggest supersonic turbulence simulation to date rely on second-order accurate finite-volume shock-capturing method implemented in the FLASH code, did not include the viscous terms, ${ }^{11}$ and boosted the grid resolution to $10,048^{3}$ in 2016 (Federrath et al., 2016). This should be compared to the first $1024^{3}$ simulation of decaying transonic turbulence with the piecewise-parabolic method (PPM) carried out in 1998 (Porter et al., 1998) to appreciate the stunning improvement in availability of computational resources achieved over the past two decades.

\subsubsection{Scaling in compressible turbulence}

Early simulations of stationary transonic turbulence at $\mathcal{M} \sim 1$ revealed a Kolmogorov-like scaling of velocity in terms of both structure functions and power spectra (Porter et al., 2002), thereby demonstrating the compatibility of the $k^{-5 / 3}$ spectrum with a mild compressibility at transonic Mach numbers. Isothermal simulations exploring supersonic regimes followed closely, but up to mid-2000s did not have enough resolution to measure the absolute scaling

\footnotetext{
11 This type of modeling is usually called an implicit large-eddy simulation (ILES) (Sytine et al., 2000) or a coarse DNS since the dissipation is of purely numerical origin and depends on the numerical method used.
} 
exponents. Instead, they had to rely on the so-called extended self-similarity hypothesis (Benzi et al., 1995) to boost the extent of the scaling range by plotting $S_{p}(r)$ against $S_{3}(r)$, which was expected to scale linearly with the lag $r$, as in the four-fifths law. This early work indicated that scaling exponents of the velocity statistics may change gradually between $\mathcal{M} \sim 1$ and $\sim 3$ as the Hausdorff dimension of the most singular dissipative structures transitions from $D \sim 1$ (vortex filaments) to $D \sim 2$ (shock waves) (Padoan et al., 2004). Thus the change in the scaling was attributed to intermittency. Higher resolution simulations showed that the velocity spectra indeed get steeper and density spectra get shallower as the turbulent Mach numbers enter the hypersonic range (Kim and Ryu, 2005; Kritsuk et al., 2006a). However, measurements of the absolute exponent $\zeta_{3}$ of the third-order velocity structure function $S_{3}(r) \propto r^{\zeta_{3}}$ at $\mathcal{M}>3$ returned $\zeta_{3} \approx 1.25$ (Kritsuk et al., 2006b; Boldyrev et al., 2002), ${ }^{12}$ indicating that the observed change in scaling could not occur due to intermittency alone.

This was a clear indication that the energy transfer in supersonic regime is mediated by density fluctuations and hence ignoring the density velocity correlations would lead to large errors in the kinetic energy flux across scales (von Weizsäcker, 1951; Lighthill, 1955; Henriksen, 1991; Fleck, 1996). A very naïve check to make was to consider the third-order structure function of a density-weighted velocity, $\boldsymbol{v} \equiv \rho^{1 / 3} \boldsymbol{u}$, which happened to be linear (Kritsuk et al., 2007a). The linear scaling at high Mach numbers was then independently confirmed in Kowal and Lazarian (2007); Schmidt et al. (2008); Schwarz et al. (2010); Zrake and MacFadyen (2012). These results were consistent with simple Richardson-Kolmogorov-like energy cascade phenomenology modified to account for density fluctuations, $\Pi(r) \sim \rho_{r} u_{r}^{3} / r \sim \rho_{0} \epsilon$, and hence triggered a quest for exact relations describing the energy cascade in compressible turbulence.

Note that the density-velocity correlations are also present at subsonic turbulent Mach numbers, when the compressibility is weak, but their effects are so small that the power spectra of $\boldsymbol{u}$ and $\boldsymbol{v}$ are practically indistinguishable (Kritsuk et al., 2007b; Wang et al., 2012). At the same time, physics of highly compressible turbulence in molecular clouds stimulated numerical experiments at high Mach numbers, which eventually led to the discovery of new fundamental scaling laws.

The first non-trivial step needed to extend Kolmogorov's phenomenology to compressible turbulence was the interpretation of the four-fifth law; namely, whether the velocity, $\boldsymbol{u}$, should be carried over to the compressible case as velocity or as momentum, $\boldsymbol{j} \equiv \rho \boldsymbol{u}$ (or as both).

Taking the latter approach, Falkovich et al. (2010) derived a series of new exact relations for fluxes and densities of conserved variables. A particular case relevant to the cascading of mean squared momentum, $\left\langle|\boldsymbol{j}|^{2} / 2\right\rangle$, injected by external forcing, produced the following exact law: $\nabla_{r} \cdot\left\langle\left(\boldsymbol{j} \cdot \boldsymbol{j}^{\prime}\right) \boldsymbol{u}^{\prime}+c_{\mathrm{s}}^{2} \rho^{\prime} \boldsymbol{j}\right\rangle=$

\footnotetext{
12 A similar slope was also obtained in simulations by W.-C. Müller (2005, private communication).
} 
$-\left\langle\rho \rho^{\prime}\right\rangle \epsilon$. While this relation reduces to the four-fifths law in the incompressible limit, it does not yield a desired universal scaling law for compressible turbulence. Indeed, it can be readily seen that scaling of the density autocorrelation function in the r.h.s. varies with the Mach number, as does the slope of the density power spectrum (Kim and Ryu, 2005), see also Wagner et al. (2012); Kritsuk et al. (2013b). One can also argue that $\boldsymbol{j}^{2}$ is not a conserved quantity and hence plays no obvious dynamically important role in compressible turbulence (Eyink and Drivas, 2018). Quite a variety of anomalous balance relations for compressible turbulence can be derived. Even though they recover the von Kármán-Howarth-Monin relation in the incompressible limit, most of them may appear physically irrelevant.

\subsubsection{Total energy as an ideal invariant}

A more traditional interpretation of the four-fifths law in terms of the kinetic energy transfer poses its own challenges when an extension to the compressible case is attempted. First, the kinetic energy is not an ideal invariant of the compressible system, but instead the total energy, $E=K+U=\langle\rho \boldsymbol{u} \cdot \boldsymbol{u} / 2+\rho e\rangle$, is invariant. Second, both kinetic, $K$, and internal, $U$, energy terms are not quadratic. Finally, in the isothermal case, the specific free energy per unit mass $e=c_{\mathrm{s}}^{2} \ln \left(\rho / \rho_{0}\right)$ is not sign-definite. Note that including the fluctuations of magnetic field, $\boldsymbol{b}$, would further add the magnetic energy, $M$, to the invariant, $E=K+M+U=\langle\rho \boldsymbol{u} \cdot \boldsymbol{u} / 2+\boldsymbol{b} \cdot \boldsymbol{b} / 8 \pi+\rho e\rangle$, and this new term is quadratic. Same is true for including the effects of self-gravity represented by the fluctuations of the free-fall acceleration, $\boldsymbol{g}$, which adds a negative quadratic contribution of the potential energy, $-W$, to the total energy, $E=K+M+U-W=$ $\langle\rho \boldsymbol{u} \cdot \boldsymbol{u} / 2+\boldsymbol{b} \cdot \boldsymbol{b} / 8 \pi+\rho e-\boldsymbol{g} \cdot \boldsymbol{g} / 8 \pi G\rangle$. The full self-gravitating MHD system would also include the magnetic induction equation and the Poisson equation, which do not add new nonlinearities.

\subsubsection{Kinetic energy spectra and correlation functions}

Let us first consider the kinetic energy, $K=\langle\rho \boldsymbol{u} \cdot \boldsymbol{u}\rangle / 2$, which can be decomposed into solenoidal, dilatational, and mean components by introducing a new variable, $\boldsymbol{w} \equiv \sqrt{\rho} \boldsymbol{u}$, and applying Helmholtz decomposition, $\boldsymbol{w}=\boldsymbol{w}_{s}+\boldsymbol{w}_{d}+\boldsymbol{w}_{o}$ (Kida and Orszag, 1990, 1992; Miura and Kida, 1995). The kinetic energy is then the sum of these three components $K=\tilde{K}_{s}+\tilde{K}_{d}+\tilde{K}_{o}$, where $\tilde{K}_{s}=$ $\left\langle\boldsymbol{w}_{s}^{2}\right\rangle / 2, \tilde{K}_{d}=\left\langle\boldsymbol{w}_{d}^{2}\right\rangle / 2$, and $\tilde{K}_{o}=\left\langle\boldsymbol{w}_{o}^{2}\right\rangle / 2$. The spectrum of kinetic energy can be defined in terms of the Fourier transform of $\boldsymbol{w}$ as $\tilde{K}(\boldsymbol{k})=|\widehat{\boldsymbol{w}}(\boldsymbol{k})|^{2} / 2$, and in the isotropic case $\tilde{K}(k)=4 \pi k^{2} \tilde{K}(\boldsymbol{k})$. The total kinetic energy spectrum then includes two familiar components, $\tilde{K}(k)=\tilde{K}_{s}(k)+\tilde{K}_{d}(k)$, and $K=\int_{0}^{\infty} \tilde{K}(k) d k+\tilde{K}_{o}$. This $\boldsymbol{w}$-based decomposition is not unique (Sagaut and Cambon, 2018), but convenient since it enforces the positive-definiteness of the compressive and rotational kinetic energies. For that reason, $\boldsymbol{w}$ has been often used to compute the kinetic energy spectrum in compressible turbulence (e.g. Cook and Zhou, 2002; Wang et al., 2013; Grete et al., 2017; Mittal and 
Girimaji, 2019). Empirically, it was noticed that in supersonic turbulence $\tilde{K}(k)$ shows a very strong "bottleneck" (present in both $\tilde{K}_{s}(k)$ and $\tilde{K}_{d}(k)$ spectra), which can be traced to shock fronts (section 3.5 in Kritsuk et al., 2007a). While the linear momentum, $\boldsymbol{j}$, is continuous across shock fronts, any massweighted velocity, $\rho^{\alpha} \boldsymbol{u}$, with $\alpha \neq 1$ is discontinuous. It is therefore likely that strong small-scale autocorrelation of $\boldsymbol{w}$ caused by the fractional weight $\rho^{\alpha}$ with $\alpha=1 / 2$ is responsible for the "bottleneck" in $\tilde{K}(k)$. Note that the compression factors across isothermal shocks can be very high $\left(\rho_{2} / \rho_{1} \sim \mathcal{M}_{\text {shock }}{ }^{2}\right)$ and therefore the effect can be strong in supersonic regimes. In contrast, in subsonic turbulence, both $\boldsymbol{u}$ - and $\boldsymbol{w}$-based decompositions yield similar results, making mass weighting impractical.

Similar effects of symmetric mass weighting appear in the scaling of third order moments (Kritsuk et al., 2013b). While the mixed structure function $\left\langle(\delta \boldsymbol{j} \cdot \delta \boldsymbol{u}) \delta \boldsymbol{u}_{\|}\right\rangle$showed a clean extended linear scaling range, $\left\langle\left|\delta \boldsymbol{v}_{\|}\right|^{3}\right\rangle$ displayed only an approximately linear scaling overall with no clear linear range (fig. 6 in Kritsuk et al., 2013b). The failure of the $\boldsymbol{v}$-based approach to capture the desired universal scaling in simulations of supersonic turbulence suggests that fractional mass weighting should be avoided.

Generally speaking, there is no fundamental a priori reason to favor one definition of the kinetic energy correlation function over another. The problem we are facing here is similar to the problem of statistical averaging in variable density fluid turbulence (see e.g. chapter 5 in Chassaing et al., 2002) and it is not yet clear what will eventually represent the best way forward. However, some guidance can be found in numerical simulations.

Similar issues arise when coarse-graining is used instead of point-splitting ${ }^{13}$ as a convenient regularization that removes short-distance divergences. The coarse-graining approach was pursued by Aluie (2011); Aluie et al. (2012); Aluie (2013) and more recently by Eyink and Drivas (2018) also to yield an analogue to Kolmogorov's four-fifths law for compressible fluid turbulence. The Favre scale-decomposition framework (Favre, 1983) separates the "resolved" kinetic energy, $\frac{1}{2}\langle\boldsymbol{j}\rangle_{r}^{2} /\langle\rho\rangle_{r} \leq \frac{1}{2}\langle\boldsymbol{j} \cdot \boldsymbol{u}\rangle_{r}$, from the "unresolved" or "subscale" kinetic energy represented by a second-order Favre cumulant, $\frac{1}{2}\left[\langle\boldsymbol{j} \cdot \boldsymbol{u}\rangle_{r}-\right.$ $\left.\langle\boldsymbol{j}\rangle_{r}^{2} /\langle\rho\rangle_{r}\right]$. When the coarse-graining operation ${ }^{14}\langle\cdot\rangle_{r}$ is applied to scale $r$ that falls in the inertial range, the cumulant (or the so-called "subscale stress") is interpreted as an effective inertial range dissipation of kinetic energy (Eyink and Drivas, 2018).

However, the mass-weighted Favre filtering is not the only way to decompose scales and regularize the equations. Alternatives include (but are not limited to): $\frac{1}{2}\langle\rho\rangle_{r}\langle\boldsymbol{u}\rangle_{r}^{2}, \frac{1}{2}\langle\boldsymbol{w}\rangle_{r}^{2}, \frac{1}{2}\langle\boldsymbol{j}\rangle_{r} \cdot\langle\boldsymbol{u}\rangle_{r}, \frac{1}{2}\langle\rho\rangle_{r}\left\langle|\boldsymbol{u}|^{2}\right\rangle_{r}$, or linear combina-

\footnotetext{
13 In this context, point-splitting regularization refers to the use of two-point statistics (such as correlation functions, structure functions, and power spectra) when the products of fields at the same spatial (temporal) location are not mathematically well-defined.

14 The coarse-graining operation is a simple convolution $\langle\boldsymbol{a}\rangle_{r}(\boldsymbol{x}, t)=\int \phi_{r}(\boldsymbol{y}) \boldsymbol{a}(\boldsymbol{x}+\boldsymbol{y}, t) d \boldsymbol{y}$ with a smooth mollifier $\phi_{r}(\boldsymbol{y})=\phi(\boldsymbol{y} / r) / r^{3}$ such that $\int \phi_{r}(\boldsymbol{y}) d \boldsymbol{y}=1$. This type of smooth filtering with compact support in space is used to single out the large scale component of a field variable $\boldsymbol{a}(\boldsymbol{x}, t)$ corresponding to length scales $>r$.
} 
tions thereof. Based on the so-called inviscid criterion (Aluie, 2013), Zhao and Aluie (2018) showed that the first two decompositions (Reynolds- and $\boldsymbol{w}$-based) fail to capture the inertial range, if density variations are significant. The key idea behind the inviscid criterion is that the scale-decomposition of momentum and kinetic energy should guarantee that viscous contributions are negligible at large enough length-scales, which is necessary for the study of inertial range dynamics. The numerical evidence presented by Zhao and Aluie (2018) is consistent with similar indications from point-splitting approach that we discussed above. This can explain the difficulty of $\boldsymbol{w}$-based point-splitting analysis in Grete et al. (2017) and Schmidt and Grete (2019) to capture a reasonably extended inertial range.

Note that the so-called transport-selected regrouping (or mixed-weighted decomposition introduced by Chassaing, 1985), using Reynolds averaging for the transporting agent (e.g. the advection velocity) and Favre averaging for the convected function (e.g. a momentum component), $\frac{1}{2}\langle\boldsymbol{j}\rangle_{r} \cdot\langle\boldsymbol{u}\rangle_{r}$, has yet to be evaluated with respect to the inviscid criterion. However, its point-splitting counterpart is known to yield robust inertial range in supersonic turbulence (Kritsuk et al., 2013b).

Getting back to the point-splitting approach, let us consider an alternative definition of the kinetic energy correlation function, $K(\boldsymbol{r})=R_{\boldsymbol{j} \boldsymbol{u}}(\boldsymbol{r}) / 2$, using the symmetric part of the cross-covariance of linear momentum density and velocity, $R_{\boldsymbol{j} \boldsymbol{u}}(\boldsymbol{r}) \equiv\langle[\boldsymbol{j}(\boldsymbol{x}) \cdot \boldsymbol{u}(\boldsymbol{x}+\boldsymbol{r})+\boldsymbol{j}(\boldsymbol{x}+\boldsymbol{r}) \cdot \boldsymbol{u}(\boldsymbol{x})]\rangle / 2$. It was used by Graham et al. (2010); Galtier and Banerjee (2011); Banerjee and Galtier (2013, 2014, 2017); Andrés and Sahraoui (2017); Banerjee and Kritsuk (2017); Andrés et al. (2018a); Banerjee and Kritsuk (2018); Andrés et al. (2018b) to derive exact relations for energy transfer in compressible turbulence analogous the von Kármán-Howarth-Monin relation we introduced earlier. This approach does not suffer from artificial "bottleneck" in the kinetic energy cospectrum, $K(\boldsymbol{k})=\widehat{R_{\boldsymbol{j} \boldsymbol{u}}}(\boldsymbol{k}) / 2=\left[\widehat{\boldsymbol{j}}(\boldsymbol{k}) \cdot \widehat{\boldsymbol{u}}^{*}(\boldsymbol{k})+\widehat{\boldsymbol{j}}^{*}(\boldsymbol{k}) \cdot \widehat{\boldsymbol{u}}(\boldsymbol{k})\right] / 4$ since linear momentum decouples from the velocity at shock fronts. ${ }^{15}$ It also recovers the detailed small-scale magnetic-kinetic energy equipartition, $K(k) \approx M(k)$, in an $1024^{3}$ MHD turbulence simulation at $\mathcal{M} \approx 10$ and plasma $\beta_{0}=2$, previously plagued by a large excess in the kinetic energy spectrum, $\tilde{K}(k)$, computed using the Fourier transform of $\boldsymbol{w}$ (e.g. fig. $3 \mathrm{~g}$ in Kritsuk et al., 2009).

\subsubsection{Internal energy spectra and correlation functions}

The same diversity of formally allowed definitions equally applies to the twopoint correlation function of thermodynamic energy. A set of options to split the non-quadratic combination of $\rho e$ betwen points $\boldsymbol{x}$ and $\boldsymbol{x}^{\prime}=\boldsymbol{x}+\boldsymbol{r}$ discussed in the literature includes: (i) $\left\langle\rho e^{\prime}\right\rangle$ (Galtier and Banerjee, 2011; Banerjee and

\footnotetext{
15 Here, ${ }^{*}$ denotes the complex conjugate and we used the convolution theorem to cast the Fourier transform of the correlation function $R_{\boldsymbol{j} \boldsymbol{u}}(\boldsymbol{r})$ using the Fourier transforms of $\boldsymbol{j}$ and $\boldsymbol{u}$. Using the symmetric cross-covariance makes sure that the spectral kinetic energy density, $K(\boldsymbol{k})$, is real. Finally, it follows from Parseval's theorem that $K=\int K(\boldsymbol{k}) d \boldsymbol{k}=R_{\boldsymbol{j} \boldsymbol{u}}(0) / 2$.
} 
Galtier, 2013, 2017; Andrés and Sahraoui, 2017; Andrés et al., 2018a; Banerjee and Kritsuk, 2018; Andrés et al., 2018b), (ii) $\left\langle\rho c_{\mathrm{s}} c_{\mathrm{s}}^{\prime}\right\rangle / \gamma(\gamma-1)$ (Banerjee and Galtier, 2014), and (iii) $\left\langle\sqrt{\rho} c_{\mathrm{s}} \sqrt{\rho^{\prime}} c_{\mathrm{s}}^{\prime}\right\rangle / \gamma(\gamma-1)$ (Schmidt and Grete, 2019; Mittal and Girimaji, 2019). The most popular option (i) uses point splitting between a conserved variable $\rho$ and the specific thermodynamic energy e. Option (ii) mimics the momentum-velocity point splitting for the kinetic energy by replacing the velocity $\boldsymbol{u}$ with the sound speed $c_{\mathrm{s}}$. It was suggested for systems with polytropic turbulence and cannot be applied to isothermal fluids where $c_{\mathrm{s}}$ is constant. Option (iii) is analogous to the $\boldsymbol{w}$-based splitting of the kinetic energy correlator, where the velocity is also replaced by the sound speed.

In contrast to the kinetic energy correlation function, where centering of the velocity and momentum $(\langle\boldsymbol{u}\rangle=0,\langle\boldsymbol{j}\rangle=0)$ can be achieved by a proper choice of the reference frame, the thermodynamic energy requires an appropriate decomposition of turbulent fluctuations from uniform background. This issue, overlooked in the early works on energy transfer in isothermal compressible turbulence, was exposed in (Banerjee and Kritsuk, 2017), where the thermodynamic energy correlation function was defined as $R_{\rho e}(\boldsymbol{r})=\langle\rho e\rangle / 2+\left\langle\rho e^{\prime}+\right.$ $\left.\rho^{\prime} e\right\rangle / 4$. In the single-point limit, we get $R_{\rho e}(0)=\langle\rho e\rangle$, as needed, and the first term in $R_{\rho e}(\boldsymbol{r})$ describes a single-point contribution required by the presence of a nontrivial uniform background in the homogeneous case. It can be readily shown with data from numerical simulations that using $\tilde{R}_{\rho e}(\boldsymbol{r})=\left\langle\rho e^{\prime}+\rho^{\prime} e\right\rangle / 2$ instead of $R_{\rho e}(\boldsymbol{r})$ would break the acoustic energy equipartition Sarkar et al. (1991) at small scales, where turbulence is strongly dominated by the acoustic mode (Falkovich and Kritsuk, 2017; Kritsuk, 2019). Moreover, incorrect uniform background removal adds spurious source terms to the scale-by-scale energy balance equation (Kritsuk and Banerjee, 2020).

We can now use $R_{\rho e}(\boldsymbol{r})$ to define the thermodynamic energy spectral density for an isothermal fluid as a cospectrum of $\rho$ and $e, U(\boldsymbol{k})=\widehat{R_{\rho e}}(\boldsymbol{k})=$ $\left[\widehat{\rho}(\boldsymbol{k}) \widehat{e}^{*}(\boldsymbol{k})+\widehat{\rho}^{*}(\boldsymbol{k}) \widehat{e}(\boldsymbol{k})\right] / 4$. From Parseval's rule, we have for the thermodynamic energy of fluctuations: $U=\int U(\boldsymbol{k}) d \boldsymbol{k}$.

\subsubsection{Scale-by-scale energy balance and energy cascades}

As a simple example, let us consider energy cascade in compressible homogeneous isothermal turbulence. The relevant ideal invariant in this case is the total energy, $E=K+U=\left\langle\rho \boldsymbol{u}^{2} / 2+\rho e\right\rangle$, where $e=c_{\mathrm{s}}^{2} \ln \left(\rho / \rho_{0}\right)$. The energy spectral density of turbulent fluctuations is given by $K(k)$ and $U(k)$ defined above. To describe the scale-by-scale energy balance, one can use the same equation we introduced for the incompressible case, $\partial_{t} E(k)=T(k)-D(k)+F(k)$, but with the transfer, forcing, and dissipation functions taken from Banerjee and Kritsuk (2017). Similar equations can be written for the kinetic and thermodynamic energy balance, but these will include the energy exchange (cross) terms, $X(k)$, which cancel out in the total energy balance: $\partial_{t} K(k)=$ $T_{K}(k)-D(k)+F(k)-X_{K \rightarrow U}(k)$ and $\partial_{t} U(k)=T_{U}(k)+X_{K \rightarrow U}(k)$. The total transfer function $T(k)=T_{K}(k)+T_{U}(k)$. The cross-scale total energy 
flux is defined in a familiar way, $\Pi(k)=\int_{k}^{\infty} T(\kappa) d \kappa$, and also includes two components, $\Pi(k)=\Pi_{K}(k)+\Pi_{U}(k)$.

This formalism can be readily used to analyze data from simulations of stationary homogeneous compressible turbulence (e.g. Falkovich and Kritsuk, 2017; Kritsuk, 2019; Kritsuk and Banerjee, 2020) and similar analysis can also be carried out in the configuration space (e.g. Kritsuk et al., 2013b, 2015). By computing statistics of $T(k), D(k)$, and $F(k)$ from the simulation data, one can get direct access to detailed picture of energy injection, transfer, and dissipation across scales, including the limits on the inertial range, direction of the cascade, possible coexistence of several independent energy cascades, etc. Unique physical definitions of relevant spectral energy densities (or correlation functions) provide means to explore detailed equipartition between various energy components of turbulent fluctuations. It is worth noting that traditional power spectra of density, velocity, and weighted velocity (e.g. $\boldsymbol{v}$ or $\boldsymbol{w}$ ) do not bear a large fraction of that information.

Including self-gravity of the gas and magnetic fields in this analysis does not pose any major technical challenges since both magnetic and gravitational potential energy components are quadratic and Poisson's equation is linear (see Banerjee and Kritsuk $(2017,2018)$, where the corresponding formalism is developed). It is worth noting that fluctuations of $\boldsymbol{g}$ and $\boldsymbol{b}$ are correlated with other fluctuating quantities in compressible turbulence, hence it is conceptually incorrect to talk about turbulence, gravity, and magnetic fields as separate factors regulating star formation in turbulent molecular clouds. It should be also mentioned that application of virial theorem (McKee and Zweibel, 1992) to such clouds (or clumps within them), while ignoring the surface terms (Dib et al., 2007), would lead to confusing results (Ballesteros-Paredes, 2006; Kritsuk et al., 2013a) since this is equivalent to setting $\Pi(r)=0$ at the cloud size scale $r$. Thus the whole concept of virial equilibrium in the context of turbulent molecular clouds or their substructure should be taken with a grain of salt. Instead, the formalism describing energy transfer in self-gravitating MHD turbulence developed in (Banerjee and Kritsuk, 2017, 2018; Kritsuk et al., 2017) should be used. It shows that the role of self-gravity of the gas can be two-fold: (i) it can provide kinetic energy injection in a wide range of scales (wide-band forcing) and (ii) it can work as a trigger of dynamic gravitational collapse at small scales, where collapsing objects decouple from the general turbulent field and the dilatational velocity component $\boldsymbol{u}_{d}$ gets locally enslaved by the gravitational acceleration $\boldsymbol{g}$, leading to small-scale equipartition of the kinetic energy and gravitational potential energy of the collapsing material, $K(k) \sim W(k)$ at $k>k_{\text {crit }}$ (Banerjee and Kritsuk, 2017).

\subsection{Bibliographical notes}

Our discussion of interstellar turbulence merely provides an overview of the recent progress achieved in the last decade, while the reader is referred to recent books and review articles on the subject. Excellent reviews on interstellar 
turbulence can be found in Elmegreen and Scalo (2004) and in Chapter 13 of Lequeux (2005); recent accounts of supersonic turbulence in the star formation context are given in Mac Low and Klessen (2004); McKee and Ostriker (2007); Hennebelle and Falgarone (2012); Padoan et al. (2014); Federrath (2018); The role of magnetic fields in molecular cloud formation and evolution is reviewed in Hennebelle and Inutsuka (2019). For general theoretical background on incompressible turbulence, see Frisch (1995); for magnetohydrodynamic (MHD) turbulence, see Biskamp (2003); Beresnyak and Lazarian (2019); for an upto-date overview of compressible turbulence, see Chapters 2, 3, and 13-16 in Sagaut and Cambon (2018) and a technical perspective in Chen et al. (2015). Current status of research in MHD turbulence theory and numerical experiments including supersonic MHD turbulence is covered in Beresnyak (2019); Lazarian et al. (2020); recent work on compressibility effects in molecular cloud and MHD turbulence is reviewed in Galtier (2018). Finally, Alexakis and Biferale (2018) is an excellent introduction to cascades and transitions in turbulent fluid flows which are not exactly homogeneous and isotropic, presenting new opportunities for addressing interesting situations in astrophysics, including large-scale turbulence in galactic disks.

\section{Magnetic fields}

\subsection{Introduction and observational facts}

Galaxies and thus the interstellar medium in galaxies are permeated by magnetic fields (Fletcher et al., 2011; Beck, 2015). First observational evidence for the magnetisation of the ISM dates back to Hall (1949), Hiltner (1949), and Davis and Greenstein (1951), who found that polarization of starlight seems to increase with reddening and to be correlated spatially, in polarization fraction and even more so angle. More recent observations of the interstellar medium and star forming regions allow to quantify the field strength and geometry (Crutcher, 1999; Bourke et al., 2001; Heiles and Crutcher, 2005; Troland and Crutcher, 2008; Crutcher, 2012; Beck, 2015; Haverkorn, 2015; Planck Collaboration et al., 2018). Using polarised synchrotron of electrons, thermal dust emission as well as starlight polarization in extinction, we are able to reconstruct the orientation of the magnetic field in the plane of the sky. The strength can be determined using Faraday rotation and the Zeeman effect, where the former one is mainly used on galactic scales and the diffuse ISM, whereas the latter one is applied in determining the field strength in dense clouds. Observations on galactic scales reveal large-scale fields, which follow the spiral structure of galactic arms (Beck, 2009; Fletcher et al., 2011). The field strength of this coherent magnetic field component ranges from a few up to a few tens of $\mu \mathrm{G}$ (Fletcher et al., 2011). In the diffuse interstellar medium fields with intensities of 0.1 to $10 \mu \mathrm{G}$ have been observed (Crutcher, 2012) with very little correlation of the field strength with gas density. For column densities above $10^{22} \mathrm{~cm}^{-2}$ the scatter remains large between individual measurements 
but there is a clear tendency of the maximum field strength to increases up to the $\mathrm{mG}$ regime. The scaling of the maximum field strength is consistent with the field compression in the ideal MHD approximation, which we will further explain below. The formation of stars is thus likely to be tightly connected to the evolution of magnetic fields. Recent reviews by Hennebelle and Inutsuka (2019) and Krumholz and Federrath (2019) focus on the impact of magnetic fields from molecular clouds down to the formation of stars and the resulting initial stellar mass function. The review by Wurster and Li (2018) explicitly discusses magnetized protostellar discs. In the following we provide a theoretical background and some basic implications of how magnetic fields influence the star formation process.

\subsection{Theoretical background}

\subsubsection{Magneto-hydrodynamics}

The basic equations describing electro-magnetism are Maxwell's equations. In Gaussian cgs units their differential form reads

$$
\begin{aligned}
4 \pi \boldsymbol{j}+\partial \boldsymbol{E} / \partial t & =c \boldsymbol{\nabla} \times \boldsymbol{B} \\
\partial \boldsymbol{B} / \partial t & =-c \boldsymbol{\nabla} \times \boldsymbol{E} \\
\boldsymbol{\nabla} \cdot \boldsymbol{E} & =4 \pi \rho_{\mathrm{e}} \\
\boldsymbol{\nabla} \cdot \boldsymbol{B} & =0
\end{aligned}
$$

Here, $\boldsymbol{E}$ and $\boldsymbol{B}$ are the electric and magnetic field vector, $\boldsymbol{j}$ is the electrical current density and $\rho_{\mathrm{e}}$ the charge density. Equation (34) is Ampère's circuital law, which relates the magnetic field around a closed loop to the electric current passing through the loop. Equation (35) is known as Faraday's law of induction. Gauss' law in equation (36) describes the charge density as the source of the electric field. And finally, Equation (37) describes the magnetic field to be source-free.

Many astrophysical systems are strongly electrically conducting, and so is the interstellar medium. In the limit of an infinite conductivity, this results in an effectively vanishing electric field. Any small electric field would immediately result in a strong current until the electric field has vanished. We note that the electric field only vanishes in the comoving frame of the fluid. An observer moving relative to the fluid with a speed $v$ could observe an electric field,

$$
\boldsymbol{E}=-\boldsymbol{v} \times \boldsymbol{B} / c .
$$

The magnetic field $(\boldsymbol{B})$ and inducition $(\boldsymbol{H})$ are related via the dimensionless relative permeability $\mu, \boldsymbol{B}=\mu \boldsymbol{H}$. Similarly, the electric field $(\boldsymbol{E})$ and the electric displacement $(\boldsymbol{D})$ are connected by relative permittivity $\epsilon$, so $\boldsymbol{D}=\epsilon \boldsymbol{E}$. In most astrophysical applications and so in the ISM both proportionality parameters are very close to unity, which allows to ignore the distinction between 
magnetic field strength and magnetic induction as well as between electric field and electric displacement.

Combining equations (35) and (38) yields the induction equation

$$
\frac{\partial \boldsymbol{B}}{\partial t}=\boldsymbol{\nabla} \times(\boldsymbol{v} \times \boldsymbol{B}),
$$

which describes the changes of the magnetic field in the presence of a velocity field $\boldsymbol{v}$ in a perfectly conducting fluid. This approximations is known as ideal MHD.

In this limit of ideal MHD we can picture the magnetic field lines to be frozen in the gas flow. This allows the gas and the magnetic field to dynamically interact and transfer momentum and energy. The Lorentz force per unit volume reads

$$
\begin{aligned}
\boldsymbol{F}_{\mathrm{L}} & =\frac{1}{c} \boldsymbol{j} \times \boldsymbol{B} \\
& =\frac{1}{4 \pi}(\boldsymbol{\nabla} \times \boldsymbol{B}) \times \boldsymbol{B} \\
& =\underbrace{-\frac{1}{8 \pi} \boldsymbol{\nabla} \boldsymbol{B}^{2}}_{\text {pressure term }}+\underbrace{\frac{1}{4 \pi}(\boldsymbol{B} \cdot \boldsymbol{\nabla}) \boldsymbol{B}}_{\text {curvature term }}
\end{aligned}
$$

which is a force per unit volume of the fluid exerted on a globally electrically neutral, conducting fluid by the magnetic field. The formulation in the last line illustrates the contribution to the Lorentz force by the gradient of the magnetic pressure $\left(\boldsymbol{B}^{2} / 8 \pi\right)$ and the curvature of the field. The pressure term can be understood in analogy to thermal pressure where the field lines provide a force per area against compression. The magnetic tension is a restoring force that acts to straighten bent field lines.

The equations of motion for ideal MHD compared to hydrodynamics can be described by adding only the magnetic field to the set of equations, i.e. without reference to other components of the Maxwell's equations. In addition, the induction equation is added as a separate evolution equation for $\boldsymbol{B}$,

$$
\begin{aligned}
\frac{\partial \rho}{\partial t}+\boldsymbol{\nabla} \cdot(\rho \boldsymbol{v}) & =0 \\
\frac{\partial \rho \boldsymbol{v}}{\partial t}+\boldsymbol{\nabla} \cdot[\rho \boldsymbol{v} \boldsymbol{v}^{\mathrm{T}}+\underbrace{\left(P_{\mathrm{th}}+\frac{\boldsymbol{B}^{2}}{8 \pi}\right)}_{P_{\mathrm{tot}}} \mathrm{I}-\frac{\boldsymbol{B} \boldsymbol{B}^{\mathrm{T}}}{4 \pi}] & =\rho \boldsymbol{g} \\
\frac{\partial e}{\partial t}+\boldsymbol{\nabla} \cdot\left[\left(u+\frac{\rho \boldsymbol{v}^{2}}{2}+\frac{\boldsymbol{B}^{2}}{8 \pi}+\frac{P_{\mathrm{th}}}{\rho}\right) \boldsymbol{v}-\frac{\boldsymbol{B}(\boldsymbol{v} \cdot \boldsymbol{B})}{4 \pi}\right] & =\rho \boldsymbol{v} \cdot \boldsymbol{g} \\
\frac{\partial \boldsymbol{B}}{\partial t}-\boldsymbol{\nabla} \times(\boldsymbol{v} \times \boldsymbol{B}) & =0,
\end{aligned}
$$


with the gas density $\rho$, the gas velocity $\boldsymbol{v}$, and the gravitational acceleration $\boldsymbol{g}$. The thermal energy density is denoted by $u$, the total energy density

$$
e=u+\frac{\rho \boldsymbol{v}^{2}}{2}+\frac{\boldsymbol{B}^{2}}{8 \pi},
$$

and the thermal pressure by $P_{\mathrm{th}}$. The notation $\boldsymbol{B} \boldsymbol{B}^{\mathrm{T}}$ is the dyadic product of two vectors. The total pressure is

$$
P_{\mathrm{tot}}=P_{\mathrm{th}}+\frac{\boldsymbol{B}^{2}}{8 \pi}
$$

and we close the system with the equation of state

$$
P_{\mathrm{th}}=(\gamma-1) \rho e .
$$

\subsubsection{Non-ideal MHD effects}

The limit of ideal MHD holds for large regions of the interstellar medium, where the gas is partially ionised. The ions gyrate around the magnetic field lines with the cyclotron frequency and are tied to the field lines. Contrary, the neutrals do not experience the Lorentz force and can move independent of the magnetic field. In principle, this allows for relative motions between the ions and neutrals, which is called ion-neutral drift or ambipolar diffusion with a drift velocity

$$
\boldsymbol{v}_{\mathrm{a}} \equiv \boldsymbol{v}_{\mathrm{i}}-\boldsymbol{v}=\frac{\boldsymbol{F}_{\mathrm{L}}}{\gamma \rho \rho_{\mathrm{i}}}=\frac{1}{4 \pi} \frac{(\nabla \times \boldsymbol{B}) \times \boldsymbol{B}}{\gamma \rho \rho_{\mathrm{i}}} .
$$

Here, $\rho$ and $\rho_{\mathrm{i}}$ are the density of the neutrals and the ions, and $\gamma$ is a friction coefficient (Mouschovias and Paleologou, 1981; Balbus, 2009). However, ions and neutrals are coupled via collisions and an efficient transfer of momentum between them would effectively also couple the neutrals to the field lines and the relative drift can be small or negligible compared to other speeds in the system. How efficient this coupling is depends on the degree of ionisation. From dimensional arguments the typical time reads

$$
\tau_{\mathrm{a}}=\frac{L}{v_{\mathrm{a}}} \sim \frac{4 \pi \gamma \rho \rho_{\mathrm{i}} L^{2}}{\boldsymbol{B}^{2}}
$$

with the characteristic length at scale $L$. Using a numerical value of $\gamma=$ $3 \times 10^{13} \mathrm{~cm}^{3} \mathrm{~s}^{-1} \mathrm{~s}^{-1}$ (Draine et al., 1983) and typical values for a star forming core $\left(L \sim 0.1 \mathrm{pc}, \rho \sim 10^{-19} \mathrm{~g} \mathrm{~cm}^{-3}, \rho_{\mathrm{i}}=10^{-23} \mathrm{~g} \mathrm{~cm}^{-3}, B \sim 100 \mu \mathrm{G}\right)$ this time scale estimate yields $\sim 100 \mathrm{Myr}$, which is much longer than the turbulent crossing time $t_{\text {turb }}=L / v_{\text {turb }} \sim 0.1$ Myr for a turbulent velocity of $1 \mathrm{~km} \mathrm{~s}^{-1}$. For a lower ion density of the ions (e.g. $\rho_{\mathrm{i}}=10^{-25} \mathrm{~g} \mathrm{~cm}^{-3}$ ) and a five times stronger field both time scales become comparable. At what scale in under what conditions ambipolar diffusion becomes relevant is still debated.

Besides ambipolar diffusion we would like to mention two further processes, namely Hall drift and Ohmic resistivity, that become important only in the 
densest regions of star formation such as the in protostellar discs. We refer the reader to the review by Wurster and $\mathrm{Li}$ (2018) for details and their importance on scales below $\sim 100 \mathrm{au}$. The Hall drift described the effect when the massive particles (ions and charged grains) decouple from the magnetic field, whereas the electrons are still tight to the field lines. This results in relative motions between electrons and ions,

$$
\boldsymbol{v}_{\mathrm{H}}=\boldsymbol{v}_{\mathrm{e}}-\boldsymbol{v}_{\mathrm{i}}=-\frac{\boldsymbol{j}}{e n_{\mathrm{e}}}=-\frac{c}{4 \pi} \frac{\boldsymbol{\nabla} \times \boldsymbol{B}}{e n_{\mathrm{e}}}
$$

with the electron charge $e$ and electron number density $n_{\mathrm{e}}$.

When all charged components (ions, electrons and charged grains) decouple from the magnetic field, Ohmic resistivity, $\eta_{\mathrm{O}}$ becomes important. The induction equation can thus be extended to (e.g. Spruit, 2013; Wurster and $\mathrm{Li}, 2018$ )

$$
\frac{\partial \boldsymbol{B}}{\partial t}=\boldsymbol{\nabla} \times\left[\left(\boldsymbol{v}+\boldsymbol{v}_{\mathrm{H}}+\boldsymbol{v}_{\mathrm{a}}\right) \times \boldsymbol{B}-\eta_{\mathrm{O}} \boldsymbol{\nabla} \times \boldsymbol{B}\right] .
$$

We would like to highlight that all non-ideal MHD effects allow for a drift between magnetic field lines and the gas. During the collapse of a gas cloud the non-ideal MHD effects therefore allow for a weaker field compared to the ideal MHD approximation.

\subsubsection{Weak and strong fields}

In order to investigate the importance of the field in a dynamical system it is useful to compare quantitatively the energy density in the magnetic field with the thermal and kinetic counterpart.

The magnetization of the gas is accompanied by magnetic waves travelling through the medium. The nature of the magnetic field allows for two types of waves, namely Alfvén waves and magneto-sonic waves. In order to determine the speeds of the waves one usually considers a uniform magnetic field in a uniform background material and investigates the transport effects of small perturbations. For a mathematical derivation we refer the reader to Shu (1992) and Spruit (2013). Alvén waves are transverse and travel along the field lines because of the magnetic tension like waves travel along a string. Alfvén waves propagate at a speed of

$$
v_{\mathrm{A}}=\frac{B}{\sqrt{4 \pi \rho}}
$$

and have the property that pressure and density perturbations vanish in the derivation. This means that Alfvén waves are incompressible. Their amplitudes are perpendicular to the unperturbed magnetic field as well as to the direction of propagation.

For magneto-sonic waves the perturbations in pressure and density do not vanish. They can be regarded as the compressive counterpart in analogy to thermal sound waves in a non-magnetised fluid. Magneto-sonic waves are characterized by the angle between the magnetic field and the direction of propagation $(\cos \theta)$ as well as the ratio of thermal sound speed $c_{\mathrm{s}}$ to Alfvén speed. 
The solution of the dispersion relation in the perturbation analysis yields two different modes, which are called the fast mode with a speed

$$
u_{\mathrm{f}}=\left(c_{\mathrm{s}}^{2}+v_{\mathrm{A}}^{2}\right)^{1 / 2}
$$

and a slow mode with a speed

$$
u_{\mathrm{s}}^{2}=\frac{c_{\mathrm{s}}^{2} v_{\mathrm{A}}^{2}}{c_{\mathrm{s}}^{2}+v_{\mathrm{A}}^{2}} \cos ^{2} \theta .
$$

An often used quantity is the ratio of thermal to magnetic pressure, which is known as plasma- $\beta$

$$
\beta=\frac{P_{\mathrm{th}}}{P_{\mathrm{mag}}} \propto \frac{c_{\mathrm{s}}^{2}}{v_{\mathrm{A}}^{2}},
$$

where $c_{\mathrm{s}}$ is the speed of sound. The analogue of the sonic Mach number,

$$
\mathcal{M}=\frac{c_{\mathrm{s}}}{v_{\mathrm{A}}} .
$$

is the Alfvénic Mach number,

$$
\mathcal{M}_{\mathrm{A}}=\frac{v}{v_{\mathrm{A}}} .
$$

For $\beta \gg 1$ the effects of the magnetic field are small. Depending on the Mach number, the system is either determined by the thermal pressure $(\mathcal{M} \ll 1)$ or by the kinetic motions $(\mathcal{M} \gg 1)$. the latter one is also called the kinematic limit.

\subsection{Magnetic fields and gravity}

In the presence of gravity, it is instructive to compare gravitational and magnetic energy. For a uniform cloud with gravitational energy $E_{\mathrm{g}}=3 G M^{2} /(5 R)$ and magnetic energy $E_{\mathrm{mag}}=\boldsymbol{B}^{2} V /(8 \pi)$ the ratio

$$
\frac{E_{\mathrm{g}}}{E_{\mathrm{mag}}} \propto \frac{M^{2}}{B^{2} R^{4}} \propto\left(\frac{M}{\Phi}\right)^{2},
$$

is proportional to the square of the mass-to-flux ratio. It is important to stress the importance of flux freezing in this context. If the gas can drift perpendicular to the field lines the mass of a contracting core can increase without changing the magnetic flux. This ratio is therefore most useful in the the ideal MHD approximation. The mass-to-flux ratio is often expressed in units of the critical value

$$
\mu=\frac{M / \Phi}{(M / \Phi)_{\text {crit }}},
$$

where the critical value is (Mouschovias and Spitzer, 1976)

$$
\left(\frac{M}{\Phi}\right)_{\text {crit }}=\frac{c_{1}}{3 \pi}\left(\frac{5}{G}\right)^{1 / 2}
$$


with a numerical dimensionless parameter $c_{1} \approx 0.53$. Structures with $\mu<1$ are called magnetically subcritical, whereas $\mu>1$ refers to supercritical regions. It is important to note that the mass is a volume quantity, whereas the magnetic flux is a surface quantity, which makes the critical value to be dependent on the geometry of the region under consideration. The above estimate is based on spherical symmetry and different numerical values have been obtained for spheroids (Mouschovias and Spitzer, 1976) and thin sheets (Nakano and Nakamura, 1978).

\subsection{Field amplification and magnetic dynamo}

There are two fundamental processes that can enhance the magnetic field strength: the first being adiabatic compression (assuming flux freezing), the second being the magnetic dynamo. Whereas adiabatic compression in idealized cases can explain the observed field strength going from galactic scales down to star forming regions, it is unlikely to be the only amplification process: In particular it will not work to attain the $\mu \mathrm{G}$ fields at Galactic scales. Given the complicated (turbulent) motions it is likely that field is also amplified by dynamo processes.

We estimate the magnetic field strength based on adiabatic compression. Let us assume that the diffuse gas in molecular clouds $\left(\rho \sim 10 \mathrm{~cm}^{-3}, B \sim\right.$ $1 \mu \mathrm{G}$ ) is compressed to protostellar cloud densities of $10^{6} \mathrm{~cm}^{-3}$ in the turbulent ISM due to gravitational forces. In the case of isotropic motions the magnetic field scales as $\rho^{2 / 3}$, which would enhance the average field strength to $\sim 2 \mathrm{mG}$. This estimate is at the upper end of the observed relation but still consistent it (Crutcher, 2012).

The adiabatic compression can account locally for strong fields, but the magnetic diffusivity is not entirely zero, even if over a typical dynamical time scale the approximation of ideal MHD is valid. Slow but steady diffusion of field lines with respect to the gas flow will eventually reduce the local field strength by evolving towards a low energy configuration. In addition reconnection of the field lines will result in topological changes of the field structure that overall minimize the energy. Therefore, the dynamical interaction of the fluid flow and a resulting field amplification is needed in order to explain the observed fields. The field amplification can be split into an amplification based on simple fluid flows on the one hand and complex turbulent flows on the other hand, where the latter one is typically referred to as turbulent dynamo. In general, the field amplification and its limitations are relatively complex and we refer to Spruit (2013), Chiuderi and Velli (2015) or Brandenburg et al. (2012) for further reading. Here, we only illustrate the basic principle of the two main models of the magnetic dynamo.

small-scale dynamo : The small-scale dynamo naturally acts in plasmas, in which magnetic fields are coupled to the fluid flow. Assume a magnetic flux 
tube with length $l$ and cross section $A$ embedded in gas with density $\rho$. Conservation of mass implies $\rho A l=$ constant; conservation of magnetic flux in the ideal MHD limit forces $B A$ to remain constant. If velocity fluctuations cause the flux tube to be stretched, the length increases and if the density does not change perceptibly, the cross section decreases, which in turn causes the field strength to increase. This amplification process can continue as long as the time scale for the diffusion of field lines $\left(\tau_{\text {diff }} \sim l_{\mathrm{d}}^{2} / \eta\right)$ is larger than the dynamical time scale for stretching the flux tubes $\left(\tau_{\text {dyn }} \sim l / v\right)$. Equating the two time scales yields $l_{\mathrm{d}} \sim l R_{\mathrm{M}}^{-1 / 2}$, where the magnetic Reynolds number is defined as $R_{\mathrm{M}}=v l / \eta$. For most of the ISM and star forming regions the magnetic Reynolds number is large, so $l_{\mathrm{d}} \ll l$, so we expect efficient dynamo action in the ISM (Brandenburg et al., 2012; Subramanian, 2019).

mean field dynamo : In order to investigate the evolution of the mean field, we decompose the magnetic field into a mean and a fluctuating component, $\boldsymbol{B}=\overline{\boldsymbol{B}}+\delta \boldsymbol{B}$. The velocity field is decomposed analogously, $\boldsymbol{v}=\overline{\boldsymbol{v}}+\delta \boldsymbol{v}$. The time evolution of the mean field is then given by (e.g. Brandenburg, 2018; Subramanian, 2019)

$$
\frac{\partial \overline{\boldsymbol{B}}}{\partial t}=\nabla \times(\overline{\boldsymbol{v}} \times \overline{\boldsymbol{B}}+\mathcal{E}-\eta \nabla \times \overline{\boldsymbol{B}}),
$$

where $\mathcal{E}=\overline{\delta \boldsymbol{v} \times \delta \boldsymbol{B}}$. By choosing an appropriate closure to express $\mathcal{E}$ in terms of the mean field $\overline{\boldsymbol{B}}$ and the mean flow $\overline{\boldsymbol{v}}$, the typical growth times in disc galaxies are of the order of $10^{8}-10^{9} \mathrm{yr}$, see for example Subramanian (2019).

\subsection{Effects of magnetic fields in star-forming regions}

The dynamical impact of the magnetic field in the star formation process encompasses several aspects. On galactic scales the strength of the magnetic field is independent of the gas density (Troland and Heiles, 1986; Crutcher et al., 2010). The field follows the large scale flows of the galactic rotation and is mainly ordered on scales of hundreds of parsecs (Beck, 2009; Fletcher et al., 2011; Beck, 2012) with field strengths of a few $\mu$ G. Here, the magnetic pressure supplements the thermal pressure against gravitational compression, which slows down the formation of dense and cold gas (e.g. Hill et al., 2012), in particular molecular gas (Girichidis et al., 2018). In addition to this delay the gas structures show smoother distributions if they are magnetized (e.g. Pardi et al., 2017). The individual fragments in the ISM, the filaments and clouds are generally more massive compared to the hydro case because the field reduces the degree of fragmentation from the diffuse gas down to the first hydrostatic core (e.g. Commerçon et al., 2011). In the low-density regime, in which self-gravity is not dominating, the probability distribution function is broadened in the magnetic compared the hydrodynamic case, albeit with a generally weak global impact (Molina et al., 2012). The authors compared turbulence simulations with and without magnetic fields showing that mainly 
the low-density range of the distribution is affected. At high densities the PDF develops a powerlaw tail (Klessen and Burkert, 2001; Slyz et al., 2005; Kainulainen et al., 2009; Girichidis et al., 2014; Schneider et al., 2015) due to the strong contraction driven by self-gravity. This range is hardy affected by magnetic fields. The reduced degree of fragmentation is propagated from the scale of GMC down to the scales of protostellar cores (Commerçon et al., 2011; Peters et al., 2014).

\section{Gravity}

Concerning gravitational forces we have to consider several aspects. On galactic scales we have to account for the gravitational attraction towards the galactic midplane, which is mainly caused by the disc as a whole and more specifically the stellar disc. In the Milky Way the stellar surface density is a factor of three larger than the gas surface density. For the hot and the warm diffuse gas, self gravity is not important. Turbulent motions and gravity on galactic scales like spiral density waves generate the seeds for molecular clouds that form close to the midplane in the dense sprial arms. Cooling results in lower thermal pressure support, but the molecular clouds as a whole are mostly still not gravitationally bound. Only the densest structures in the clouds are dynamically dominated by self-gravity and start to collapse if the gravitational compression exceeds the opposing pressure forces such as magnetic and thermal contributions and rotational support. Here we focus on the basic principles of self-gravitating isothermal gas dynamics.

\subsection{Ratio of thermal and gravitational energies}

We start by computing the ratio between the thermal energy,

$$
E_{\text {therm }}=\frac{M}{(\gamma-1) m_{p}} k_{\mathrm{B}} T \propto R^{3} P \propto R^{3} \rho^{\Gamma} \propto R^{3-3 \Gamma}
$$

and the gravitational counterpart $E_{\text {grav }}=-(3 / 5) M^{2} G / R$, with the cloud mass $M$, the radius $R$, the mean mass per particle $m_{p}$, the temperature $T$ and the pressure $P$. The Boltzmann constant is $k_{B}$ and the adiabatic index $\gamma$ depends on the number of internal degrees of freedom of the gas. Assuming a polytropic gas, the thermal pressure is $P=K \rho^{\Gamma}$ with $\Gamma$ being the effective adiabatic exponent including cooling processes. With this expression we find

$$
\frac{E_{\text {therm }}}{E_{\text {grav }}} \propto R^{4-3 \Gamma} .
$$

The scaling with $R$ revelas that $\Gamma=4 / 3$ is a critical case below which thermal pressure is unable to support the cloud against gravitational collapse because of a decreasing ratio of thermal support to gravitational energy with increasing radius. While this is true for the isothermal case, $\Gamma=1$, the gravitational 
collapse will be halted by thermal pressure as soon as the gas is unable to cool efficiently any more, which occurs when the gas becomes optically thick and the heating doe to compression cannot be radiated away. We note that for a monoatomic gas $\Gamma \simeq \gamma=5 / 3$ and for a diatomic one $\Gamma \simeq \gamma=7 / 5$.

6.2 Jeans length, Jeans mass and freefall time

The Jeans length (Jeans, 1902; Lequeux, 2005) is obtained via a linear analysis of the self-gravitating fluid equations. For a uniform cloud with density $\rho_{0}$, radius $R$, and sound speed $c_{\mathrm{s}}$ a linear analysis leads to the dispersion relation

$$
\omega^{2}=c_{\mathrm{s}}^{2} k^{2}-4 \pi G \rho_{0},
$$

We note however, that a self-gravitating isothermal cloud cannot have a strictly uniform density because pressure forces have to compensate for the gravitational attraction. For a wave number, $k$, smaller than $\sqrt{4 \pi G \rho_{0}} / c_{\mathrm{s}}$, the waves cannot propagate and perturbations are exponentially amplified. This threshold leads to the Jeans length,

$$
\lambda_{J}=\sqrt{\frac{\pi c_{\mathrm{s}}^{2}}{G \rho_{0}}},
$$

with the gravitational constant $G$. Physically this result means that self-gravity induces a contraction on a time scale of $1 / \sqrt{G \rho_{0}}$. Thermal pressure counteracts this contraction by reestablishing a uniform density over the time scale of a sound crossing time, $R / c_{\mathrm{s}}$. If $1 / \sqrt{G \rho_{0}}<R / c_{\mathrm{s}}$, the thermal pressure cannot erase fluctuations induced by the gravitational forces before the entire cloud collapses.

The Jeans mass is simply defined as the mass contained in a volume with a radius of the Jeans length, $\lambda_{J}$, and reads

$$
\begin{aligned}
M_{J} & =4 \pi / 3 \rho_{0}\left(\lambda_{J} / 2\right)^{3} \\
& =\frac{\pi^{5 / 2}}{6} \frac{c_{\mathrm{s}}^{3}}{\left(G^{3} \rho_{0}\right)^{1 / 2}} .
\end{aligned}
$$

We note that there is no fundamental justification for this choice within a factor of a few.

Equation (62) indicates that the Jeans mass decreases with increasing density, assuming an isothermal equation of state. Consequently, during the collapse of a region of a given mass the number of Jeans masses increases as the collapse proceeds. Hoyle (1953) used this argument to propose the concept of recursive fragmentation by which a cloud continues to fragment into more and more condensations as the density increases. However, as shown by eq. (60), the growth rate of the gravitational instability decreases with increasing $k$, (since $\omega^{2}<0$ ). This means that perturbations at large scales evolve faster than their small scale counterparts. As a result, the recursive fragmentation 
scenario suffers a timescale problem. A perturbation analysis of the exact solutions of the hydrostatic equilibrium reveals that the growth rate tends to zero for $k \rightarrow 0$ and the fastest growing mode corresponds to a few times the Jeans length (e.g., Nagai et al. 1998 for layers or Fiege and Pudritz 2000 for filaments). This solves the aforementioned time scale issue.

Generally, a solution for a cloud to collapse cannot be computed analytically. However, in the limit of spherical cold cloud of uniform density with a vanishing pressure one can calculate the collapse time exactly, which yields the free-fall time (see e.g., Lequeux, 2005),

$$
\tau_{\mathrm{ff}}=\sqrt{\frac{3 \pi}{32 G \rho_{0}}}
$$

6.3 The smallest Jeans mass in contemporary molecular clouds

The derivation of the Jeans mass in eq. (62) assumes a barotropic equation of state (i.e. the pressure is solely a function of density), which includes isothermal fluids as a particular case, and neglects explicit heating and cooling processes. The hierarchy of fragmentation as mentioned above in (close to) isothermal conditions will halt if the gas is so optically thick or the collapse so fast that the $P d V$ work released during the contraction cannot be radiated away any more. This limit determines the value of the smallest Jeans mass. We follow the approach of Rees (1976) and Whitworth et al. (2007) to compute this mass limit. Two conditions must be fulfilled. The first requires the size of the condensation, $R$, to be of the order of the Jeans length as explained above. The condition $R \simeq \lambda_{J}$ leads to

$$
R \simeq \frac{6}{\pi^{2}} \frac{G}{c_{\mathrm{s}}^{2}} M_{J}
$$

The second condition requires the energy released through gravitational contraction to be efficiently radiated. If this condition is not fulfilled the effective adiabatic index, $\Gamma$, will be larger than $4 / 3$ and thermal pressure will halt the collapse. The heating rate is given by the work of the thermal pressure per unit time $P d V / d t$. For a collpse in approximately freefall, we find $v=d R / d t \simeq \sqrt{2 G M / R}$ and therefore

$$
-P \frac{d V}{d t}=-\rho c_{\mathrm{s}}^{2} \frac{d}{d t}\left(\frac{4 \pi}{3} R^{3}\right) \simeq \frac{3 c_{\mathrm{s}}^{2} M}{R} \sqrt{\frac{G M}{R}} .
$$

The cooling due to radiative losses in the optically thick regime is given by (e.g. Mihalas and Mihalas, 1984; Hansen et al., 2004; Whitworth et al., 2007)

$$
\mathcal{L}=\frac{4 \pi R^{2} \sigma T^{4}}{\tau_{\text {eff }}}
$$


where $\tau_{\text {eff }}$ is the effective optical depth and $\sigma=2 \pi^{5} k_{\mathrm{B}}^{4} / 15 h^{3} c^{2}$ the StefanBoltzmann constant. In order to be ravitationally unstable heating and cooling need to balance,

$$
-P \frac{d V}{d t} \simeq \frac{3 c_{\mathrm{s}}^{2} M}{R} \sqrt{\frac{G M}{R}} \simeq \mathcal{L}=\frac{4 \pi R^{2} \sigma T^{4}}{\tau_{\mathrm{eff}}} .
$$

Solving for the radius yields

$$
R \simeq\left(\frac{3^{4} 5^{2}}{2^{8} \pi^{12}}\right)^{1 / 7}\left(\frac{G h^{6} c^{4}}{c_{\mathrm{s}}^{12} m_{p}^{8}}\right)^{1 / 7} \tau_{\mathrm{eff}}^{2 / 7} M^{3 / 7},
$$

where we assume the mean molecular weight to be unity in the sound speed. Combining the conditions stated by eqs. (64) and (68), we find a characteristic mass of

$$
\begin{aligned}
M & \simeq\left(\frac{5^{2} \pi^{2}}{2^{15} 3^{3}}\right)^{1 / 4}\left(\frac{h c}{G}\right)^{3 / 2} m_{p}^{-2}\left(\frac{c_{\mathrm{s}}}{c}\right)^{1 / 2} \tau_{\mathrm{eff}}^{1 / 2} \\
& \simeq \frac{m_{\text {Planck }}^{3}}{m_{p}^{2}}\left(\frac{c_{\mathrm{s}}}{c}\right)^{1 / 2} \tau_{\text {eff }}^{1 / 2}
\end{aligned}
$$

where $m_{\text {Planck }}=\sqrt{\hbar c / G}$ is the Planck mass. The exact numerical factor depends on the detailed assumptions. For an optical depth $\tau_{\text {eff }} \simeq 1$, the minimum mass that can collapse of order a few Jupiter masses. Whitworth and Stamatellos (2006) and Masunaga and Inutsuka (2000) discuss that $\tau \geq 1$ is not a necessary condition for collapse although it appears reasonable in this context.

\subsection{Equilibrium configurations}

In equilibrium configurations the pressure forces compensate gravitational forces. These static solutions of the fluid equations are of interest in order to test numerical codes and perform more rigorous stability analyses than the Jeans analysis. In some cases they can directly be compared to observations. The equations of hydrostatic equilibrium assuming an isothermal equation of state and the Poisson equation read

$$
\begin{aligned}
-c_{\mathrm{s}}^{2} \partial_{X} \rho+\rho \partial_{X} \phi & =0 \\
\frac{1}{X^{D-1}} \partial_{X}\left(X^{D-1} \partial_{X} \phi\right) & =-4 \pi G \rho .
\end{aligned}
$$

Combining these two equations yields the so-called Lane-Emden equation,

$$
\frac{1}{X^{D-1}} \partial_{X}\left(X^{D-1} \frac{\partial_{X} \rho}{\rho}\right)=-\frac{4 \pi G}{c_{\mathrm{s}}^{2}} \rho,
$$

with the dimension $D$ and the spatial coordinate $X$. 
The one-dimensional configuration $(D=1, X$ is the usual Cartesian coordinate, $z$ ) represents the plane-parallel geometry. The analytical solution of self-gravitating layer has been investigated by Spitzer (1942). In the twodimensional cases corresponding to cylindrical coordinates with $D=2$ and $X$ being the cylindrical radius, the analytical solution of a self-gravitating filament has been obtained by Ostriker (1964). Both solutions are characterized by a flat density profile near $X=0$. At large $X$ the two solutions differ. Whereas Spitzer (1942) presents an exponential decrease of the density at large $z$, Ostriker (1964) finds a profile decreasing as $r^{-4}$.

In spherical geometry, where $D=3$ and $X$ is the spherical radius, $r$, the solutions of eq. (73) are called Bonnor-Ebert spheres (Ebert, 1955; Bonnor, 1956). In general, the equations cannot be solved analytically but must be obtained numerically. A noticeable exception is the singular isothermal sphere (SIS) whose density profile is given by $\rho_{\mathrm{SIS}}=c_{\mathrm{s}}^{2} /\left(2 \pi G r^{2}\right)$. The density profile of the Bonnor-Ebert sphere is flat in the central part and asymptotes toward the profile of the singular isothermal sphere at large radii. In order to obtain a finite radius, the solutions are obtained up to an arbitrarily defined radius, assuming pressure equilibrium diffuse and warm medium outside the cloud. A whole family of equilibrium solutions is obtained, which are parameterized by the density contrast between the central core and the integration edge. Performing a analysis reveals that solutions with a density contrast smaller than about 14 are stable, and unstable otherwise.

Stability analyses of self-gravitating layers and filaments have been performed (e.g. Larson 1985, Fiege and Pudritz 2000). Both configurations are unstable to perturbations with a wavelengths of order the Jeans length. This suggests that periodically distributed cores or filaments could form through gravitational instability within self-gravitating filaments and layers. Since the interstellar medium is not in steady state equilibrium but stirred supersonic motions, it is difficult to address this paradigm quantitatively. However, qualitatively, spatially approximately periodically distributed cores and filaments are observed (Dutrey et al., 1991; Takahashi et al., 2013; Palau et al., 2018; Lu et al., 2018).

\subsection{Gravitational collapse}

The gravitational collapse of a spherical cloud has been investigated both analytically and numerically. Analytical models have mainly focused on selfsimilar solutions (e.g. Larson, 1969; Penston, 1969; Shu, 1977; Whitworth and Summers, 1985) which allow the reduction of the non-linear equations to simpler ordinary differential equations. These solutions provide easily timedependent density and velocity fields and help to understand the physics of the collapse. There are two main types of solutions. The one by Larson (1969) and Penston (1969) describes supersonic infall velocities at large radii $\left(\simeq 3.3 c_{\mathrm{s}}\right)$. Contrary, in the solution by Shu (1977) the gas is initially at rest and collapse evolves inside-out. Starting from the centre in which the protostar forms, A 
rarefaction wave propagates outwards at the sound speed. All self-similar solutions share the property of a constant accretion rate, which is equal to a few up to several times $c_{\mathrm{s}}^{3} / G$. We note that all solutions are characterized by a density field proportional to $r^{-2}$ in the outer part of the cloud and a $r^{-3 / 2}$ profile in the central region which has been reached by the rarefaction wave. Finally, the density in the Larson-Penston solution is about 8 times larger than the one in the Shu solution at infinite radius.

There are also a few noticeable numerical solutions. Starting from an spherical cloud with initially uniform density Larson (1969) calculates the gas contraction up to the formation of the protostar including a simplified model of radiative transfer (see also Masunaga and Inutsuka, 2000). The first accretion shock in his simulation forms at the edge of the thermally supported core. This core forms when the dust becomes opaque to its own radiation, i.e. at a density of about $10^{-13} \mathrm{~g} \mathrm{~cm}^{-3}$, and is sometimes called the first Larson core. A second accretion shock develops at the edge of the protostar at significantly higher density $\left(\simeq 10^{-2} \mathrm{~g} \mathrm{~cm}^{-3}\right)$. The model by Foster and Chevalier (1993) starts with a marginally unstable Bonnor-Ebert sphere. In their solution the collapse proceeds very slowly in the outer part of the cloud and only develop subsonic infall motions. Supersonic motions only appear in the inner part of the cloud, which indeed converges towards the Larson-Penston solution. In the outer part of the envelope, the density profile remains close to solution of the SIS. Simulations of triggered collapse have also been investigated by various authors (e.g. Hennebelle et al., 2003). There, typically faster infall velocities are reached and densities a few times higher than the ones of the SIS. Contrary to the self-similar solution, the triggered numerical models all develop strongly varying accretion rate varies over time.

\section{Overview of Stellar Feedback}

Stars impact their environment through a range of energetic processes including radiation, magnetically launched outflows, winds, and supernova explosions. This stellar feedback powers a variety of cosmic processes including heavy element production, evolution of galaxies, reionization of the Universe, formation of planetary systems and ultimately the prevalence of life.

Stellar feedback acts over a broad range of physical scales, carrying mass, momentum and energy from stellar scales $(\sim$ au $)$ up to galactic scales $(\sim$ $\mathrm{kpc}$ ). Unlike the fundamental processes described above, feedback is not a single physical process but a heterogeneous set of effects that arise from the messy and energetic life cycle of stars.

In this section, we begin with the feedback from individual stars - protostellar outflows, radiation and winds - and then discuss the collective and multiplicative effect of feedback when many stars act together. It is instructive to visualize this progression as a "feedback ladder," with the various sources ordered based on their energy and scale of influence (Bally, 2011). We will begin with the lowest "rung" of the ladder: protostellar outflows. 


\subsection{Protostellar Outflows}

Stars begin to shape their environment during formation. The process of accretion is surprisingly violent, producing significant radiation (see $\S 7.2$ ) and flinging mass at velocities of $10 \mathrm{~s}-100 \mathrm{~s}$ of $\mathrm{km} / \mathrm{s}$ out to $\sim 0.1-1 \mathrm{pc}$ from the forming star in an outflow. Outflows form as a result of rotating gas that winds up the magnetic field lines. Mass coupled to the field is redirected outwards, carrying away angular momentum and thereby facilitating accretion of lower angular momentum material. Here we use the term outflow to refer to the phenomena of collimated mass-loss from young stars, while jet refers to a very narrowly collimated outflow. The first protostellar outflows were discovered in molecular emission in 1980 (Snell et al., 1980; Rodriguez et al., 1980) (see Bally (2016) for a recent detailed review of protostellar outflows). Molecular outflows, predominately observed in ${ }^{12} \mathrm{CO}$ and ${ }^{13} \mathrm{CO}$ are typically associated with the youngest and most embedded stage of star formation when the young outflow entrains a significant amount of the surrounding core envelope as it travels away from the star. The result is that molecular outflows are relatively slow moving with velocities of a few to $10 \mathrm{~s}$ of $\mathrm{km} \mathrm{s}^{-1}$ and gas temperatures of 10s of K. Older, less embedded young stellar objects are associated with highly collimated, optical jets, which achieve velocities of $200 \mathrm{~km} \mathrm{~s}^{-1}$ and commonly exhibit atomic line and maser emission (Reipurth and Bally, 2001).

It is now accepted that outflows are a fundamental part of the star formation process and a by-product of the formation of stars ranging from brown dwarfs (Lee et al., 2009; Whelan et al., 2009) to massive stars (Shepherd and Churchwell, 1996; Zhang et al., 2005; Cyganowski et al., 2008). The ubiquity of outflows suggests that a universal launching mechanism is at work.

Obscuration by dust and gas during the early stages of star formation have frustrated high-resolution observational studies of the expected $\sim a u$ launching region. Consequently, analytic models and numerical simulations have provided the primary insights into the launching mechanism. Seminal theoretical work by Blandford and Payne (1982) proposed a general theory to describe outflow launching in hydro-magnetic accreting systems, ranging from protostars to active galactic nuclei. In this model, outflows are caused by magneto-rotational coupling, whereby magnetic fields anchored to rotating accreting gas can centrifugally redirect the gas outwards along open field lines, accelerating it to high velocities. They derived a minimum critical angle of $30^{\circ}$ between the poloidal component of the magnetic field and the rotation axis for jet launching to occur. Successive work built on these principles to propose models describing outflows from young accreting stars. The "X-Wind" model developed a formulation for outflows in which the magnetic fields are anchored near the magnetospherical truncation radius of the disk, i.e. where the magnetic pressure balances the ram pressure of the accreting material, which is otherhwise known as the "X-point" (Shu et al., 1988, 1995). Due to the small launching radius, the predicted velocities are expected to be comparable to Keplerian velocity at the stellar surface, $v_{w}=\sqrt{G M_{*} / r_{*}} \sim 100-200$ $\mathrm{km} / \mathrm{s}$ with $\sim 30 \%$ of accreting material being redirected into a well-collimated 
jet. In contrast, in the "disk-wind" model, the magnetic field lines are anchored within the disk. and material is launched over a wider range of radii (Pelletier and Pudritz, 1992). Disk-winds are slower, less well collimated and expected to eject $10 \%$ of accreting material. However, the two mechanisms are not mutually exclusive. High-resolution ALMA observations find both a wide-angle component and a well-collimated, episodic component (Zhang et al., 2016, 2019), which suggests both launching mechanisms may be active simultaneously.

Numerical simulations are necessary to progress beyond idealized, stationary axisymmetric MHD outflow models and to explore the impact of initial conditions (Banerjee and Pudritz, 2006; Hennebelle and Fromang, 2008; Machida et al., 2008; Commerçon et al., 2010; Tomida et al., 2010; Price et al., 2012; Machida and Hosokawa, 2013). However, the launching velocity is sensitive to the simulation resolution (Seifried et al., 2012), where $\sim$ au resolution is required to produce even slow outflows of $10 \mathrm{~s}$ of $\mathrm{km} / \mathrm{s}$, while $R_{\odot}$ resolution is required to resolve the launching of the highest velocity material. High-resolution calculations that follow the collapse until the formation of the second core (protostar) find that outflow launching ensues as soon as a compact rotating structure forms, leading to a slow outflow of a few $\mathrm{km} / \mathrm{s}$ even before the protostar forms (Tomida et al., 2010; Price et al., 2012). Further advances in computing power are necessary to achieve higher resolution, multi-physics simulations and be able to follow the outflow evolution over star formation timescales of a few $\sim 0.1$ Myr.

Outflows impact the star formation process and molecular clouds in a variety of ways. They play an important role in setting the efficiency of star formation (Wang et al., 2010b; Hansen et al., 2012; Federrath et al., 2014; Tanaka et al., 2017), clearing the natal envelope (Offner and Arce, 2014; Zhang et al., 2016), driving turbulence both locally (Offner and Arce, 2014; Offner and Chaban, 2017) and globally (Nakamura and Li, 2007; Carroll et al., 2009; Wang et al., 2010b; Hansen et al., 2012) and transporting angular momentum (Bai et al., 2016).

\subsection{Radiation}

Stars emit a significant amount of radiation while forming. Radiation influences the surrounding gas in three main ways: heating, ionization and dynamics, namely via radiation pressure. The latter two effects are relevant only for high-mass protostars $\left(M>10 M_{\odot}\right)$. We discuss the origin of the radiation and the scope of the impact below.

Origin. During the earliest stages of protostar formation, nuclear processes have not yet started and gravitational contraction is the source of the energy ultimately emitted as radiation. During accretion, gas accelerates as it falls into the gravitational potential well of the star. It slams to a halt on the stellar surface in a strong accretion shock. The gas kinetic energy is converted to heat, most of which is radiated away. The resulting accretion luminosity 
can be expressed as (Stahler et al., 1980; Offner and McKee, 2011):

$L_{\mathrm{acc}}=f_{\mathrm{acc}} \frac{G M_{*} \dot{M}_{*}}{R_{*}} \simeq 6.2 f_{\mathrm{acc}}\left(\frac{M_{*}}{0.25 M_{\odot}}\right)\left(\frac{\dot{M}_{*}}{2 \times 10^{-6} M_{\odot} \mathrm{yr}^{-1}}\right)\left(\frac{2.5 R_{\odot}}{R_{*}}\right) L_{\odot}$,

where $M_{*}$ and $R_{*}$ are the stellar mass and radius, respectively, $\dot{M}_{*}$ is the accretion rate, and $f_{\text {acc }}$ is an efficiency factor that reflects how efficiently heat is radiated away. This factor is not well-constrained, since it depends on the properties of the shock and accretion flow (Ostriker and Shu, 1995), and it is often assumed that $f_{\text {acc }} \simeq 1$ (Hartmann et al., 2016). However, this factor is central to the details of protostellar evolution, radii and inferred ages (Baraffe et al., 2009; Hosokawa et al., 2011).

As protostars contract along the Hayashi track (the luminosity-temperature relationship followed by pre-main sequence stars on the Hertzsprung-Russell diagram) towards the main sequence, they also radiate according to internal processes, including gravitational contraction and deuterium burning. For low-mass stars, $L_{\text {int }}$ is generally negligible compared to the accretion luminosity. Once nuclear processes begin and accretion starts to decline the intrinsic luminosity contributes an increasing larger fraction of the total luminosity.

Low-mass stars do not begin to fuse hydrogen until reaching the main sequence, but they fuse deuterium once their central temperatures reach $T \simeq$ $10^{6} \mathrm{~K}$. In contrast, high-mass stars join the main sequence and begin burning hydrogen while still vigorously accreting. For stars with $M>10 M_{\odot}$, the intrinsic stellar luminosity exceeds the accretion luminosity even at high accretion rates of $\dot{M}=10^{-4} M_{\odot} \mathrm{yr}^{-1}$ (Krumholz et al., 2009a).

Heating. The emitted radiation heats the surrounding gas and the gas temperature declines are

$$
T=\left(\frac{L_{*}}{4 \pi \sigma r^{2}}\right)^{1 / 4} \simeq 70\left(\frac{L_{*}}{1 L_{\odot}}\right)^{1 / 4}\left(\frac{r}{100 \mathrm{au}}\right)^{-1 / 2} K
$$

where $r$ is the distance from the emitting source. The radiation may be beamed more asymmetrically in the presence of an accretion disk and outflow cavity, in which radiation is preferentially escapes in the polar direction where the optical depth is lower (Yorke and Sonnhalter, 2002; Krumholz et al., 2005; Robitaille, 2011). While the extent of the heating is relatively modest for low-mass stars it is sufficient to increase the stability of the accretion disk, ultimately shaping the stellar initial mass function (Offner et al., 2014).

\subsection{Stellar Winds}

Stars of all masses and ages continuously shed mass in a high-velocity wind. The high mass-loss rates of winds launched by massive OB-type stars inject significant momentum and energy into the surrounding gas (Churchwell et al., 2006), aid in cloud dispersal (Rogers and Pittard, 2013) and help to power 
galaxy evolution (van der Kruit and Freeman, 2011; Hopkins et al., 2018). The strength and character of stellar winds vary as a function of stellar type and evolutionary phase, spanning a broad range of physical mechanisms. Winds are typically described by two fundamental parameters: $\dot{M}_{w}$, the mass-loss rate or mass-loss per unit time, and $v_{\infty}$, the terminal velocity or wind velocity far from the star. We review each of the three main wind-driving mechanisms below.

Gas-Pressure Driven Winds. Coronal winds, such as that of our Sun, are powered by gas pressure. The wind is launched by an outwardly increasing temperature gradient $\sim 10^{4}$-a few $10^{6} \mathrm{~K}$ in the solar photosphere that lifts mass from the surface (Parker, 1958). All cool stars with effective temperatures $T_{\text {eff }} \lesssim 6,500 \mathrm{~K}$ and a sub-surface convection zone have winds driven by gas pressure (Lamers and Cassinelli, 1999). This includes main-sequence stars with spectral types later than F5V or post-main sequence stars with types F5IV-K1III (Lamers and Cassinelli, 1999). Although coronal winds achieve terminal velocities of hundreds of $\mathrm{km} \mathrm{s}^{-1}$, the mass-loss rates are quite low, $\dot{M}<10^{-10} M_{\odot} \mathrm{yr}^{-1}$, so the net wind momentum and impact on the surroundings is small.

Radiation-Pressure Driven Winds. O, B, and A-type main-sequence stars, giants and supergiants emit prodigious amounts of radiation with net photon momentum that drives mass-loss rates of $\dot{M} \sim 10^{-9}-10^{-4} M_{\odot} \mathrm{yr}^{-1}$ and wind velocities up to $2,000 \mathrm{~km} \mathrm{~s}^{-1}$ (Vink et al., 2001). Such winds are known as "line-driven winds," since they are mediated by optically thick spectral lines. The Doppler effect plays a key role: a velocity gradient between the photosphere and outer stellar atmosphere allows redshifted photons to be absorbed in the outer layers without undergoing significant attenuation by the intervening material thereby contributing to accelerating the outer layers.

The momentum in the wind can be related to the stellar luminosity,

$$
\dot{M} v_{\infty}=\left(L_{*} / c\right) \tau_{w}
$$

where $\tau_{w}$ is the wind optical depth (Lamers and Cassinelli, 1999). In the limit that all photons are absorbed or scattered by the wind, $\tau_{w}=1$. In reality the wind is driven by a finite number of optically thick lines, $N_{\text {eff }}$, such that the efficiency of momentum transfer is (Lamers and Cassinelli, 1999):

$$
\eta_{\mathrm{mom}}=\dot{M} v_{\infty} /\left(L_{*} / c\right) \simeq N_{\mathrm{eff}} v_{\infty} / c,
$$

which depends on the typical optical depth of the lines. Then the terminal velocity is $v_{\infty} \simeq c / N_{\text {eff }}$ for $N_{\text {eff }}$ non-overlapping lines. Observed velocities of $v_{\infty} \sim 10^{3} \mathrm{~km} \mathrm{~s}^{-1}$ attest to the large number of spectral lines contributing to wind acceleration.

Line-driven winds are sensitive to the stellar metallicity, $Z$, since wind acceleration is powered by the absorption and re-emission of UV photons by heavy ions such as $\mathrm{C}, \mathrm{N}, \mathrm{O}$, and Fe. Wind mass-loss rates scale as $\dot{M} \propto$ $Z^{m}$, where $m \simeq 0.7-0.8$ (Vink et al., 2001; Smith, 2014). The efficiency of the acceleration also depends on the fractional abundance of metal ions, line 
optical depths and the amount of small-scale density inhomogeneities, which leads to lower mass-loss rates in early-O and late-type B stars (Lucy and Solomon, 1970; Smith, 2014).

Luminous, cool stars, namely cool supergiants, exhibit high mass-loss rates; however, these stars do not have coronae and their winds are not driven by gas pressure like those of other cool stars. Instead, radiation pressure acts on small dust grains that form in relatively cool regions above the photosphere $(T \lesssim 1500 \mathrm{~K})$ (Smith, 2014). The dust opacities are significantly higher than spectral line opacities, allowing efficient acceleration. While the mass-loss rates from such cool stars are relatively high, wind velocities are slow, reaching only a few $10 \mathrm{~s}$ of $\mathrm{km} \mathrm{s}^{-1}$.

Magnetically Driven Winds. Low-mass stars, such as F-type and later, have convective outer envelopes that enable the production of magnetic waves and magnetic energy transfer. Magnetic fields in concert with rotation can boost wind mass-loss rates, momentum and energy via "magnetic rotator" winds. One side effect of magnetically driven winds is that stellar angular momentum is carried away by the wind, such that stars spin-down over time (Weber and Davis, 1967). The Sun and other low-mass stars have appreciably lower rotation rates compared to their younger counterparts due to this angular momentum transport mechanism (Kraft, 1967; Bouvier et al., 2014). Magnetic rotator winds can also act in conjunction with the wind acceleration mechanisms described above, enhancing the wind strength, especially for young fast-rotating stars ("fast magnetic rotators") (Lamers and Cassinelli, 1999).

The wind mass-loss rate depends on the magnetic field strength and the stellar rotation rate, which vary with stellar type and age. Typically, magneticallydriven wind velocities are a few hundred $\mathrm{km} \mathrm{s}^{-1}$.

\subsection{Supernovae}

Supernovae can be classified into two main types. In thermonuclear supernovae, a white dwarf experiences runaway nuclear burning after being driven over the Chandrasekhar mass by accretion from a companion star (singledegenerate case) or merging with another white dwarf (double-degenerate case, Churazov et al., 2014; Diehl et al., 2014; Jha et al., 2019). In core collapse supernovae, the core of a massive star collapses after its main sequence life (Smartt, 2009). In both cases, typical explosion energies are of the order $10^{51} \mathrm{erg}$, as inferred from the typically ejected mass $\left(1 M_{\odot}\right)$ and velocity $\left(10,000 \mathrm{~km} \mathrm{~s}^{-} 1\right)$ of the ejecta. According to a widely used historical classification dating back to Minkowski (1941) and subsequently refined, thermonuclear supernovae are mainly of spectral type Ia (no hydrogen lines), whereas core collapse supernovae are classified as type II (strong hydrogen lines).

Massive stars $\left(>8-9 M_{\odot}\right)$ explode as core collapse supernovae, but not all stars explode (Müller et al., 2016; Ebinger et al., 2019). The theoretical models indicate certain mass ranges and possibly dependence on other parameters that determine if a star explodes or collapses to a black hole without explosion. 
Stars with initially several ten solar masses that do explode may sometimes have high explosion energies, up to several times $10^{52}$ erg (Mazzali et al., 2014; Heesen et al., 2015)

\subsection{Cosmic Rays}

Cosmic rays (CRs) are high-energy particles with non-thermal spectral energy distributions (Strong et al., 2007; Grenier et al., 2015). Strong shocks can accelerate particles from the thermal bath to super-thermal energies via diffusive shock acceleration (Axford et al., 1977; Krymskii, 1977; Blandford and Ostriker, 1978; Bell, 1978; Caprioli and Spitkovsky, 2014; Marcowith et al., 2016), which makes them the most promising site for CR production. The majority of Galactic CRs are produced by shocks in SN remnants, where approximately $5-10$ percent of the SN energy can be converted to the high-energy component (Aharonian et al., 2004, 2006, 2007; Abdo et al., 2011; Ackermann et al., 2013). This fraction has been constrained using Gamma ray emission, which is emitted during the interactions of CRs with the gas in the ISM. Stellar winds also provide appropriate conditions for CR acceleration. However, the total amount of CRs produced by stellar winds is certainly less than that by $\mathrm{SNe}$ (Webb et al., 1985), and the exact number is still a matter of debate. The composition of CRs reflects to first order the abundances in the ISM with protons occupying the largest fraction (e.g. Grenier et al., 2015). The energy distribution ranges from the thermal bath up to particle energies of $10^{20} \mathrm{eV}$ (e.g. Zweibel, 2013). Most of the energy is in CR protons at an energy of a few $\mathrm{GeV}$. Particles with higher energies are much less abundant, the low-energy counterpart does not carry enough energy per particle. As a result the GeV protons are the CRs that are dynamically relevant because of two reasons. They have comparable energy densities to the magnetic, thermal and kinetic one in the ISM (Ferrière, 2001). In addition the GeV CRs efficiently interact with the magnetic field in the ISM via the streaming instability (Kulsrud and Pearce, 1969), which heats the ISM (Wiener et al., 2013) and transfers enough momentum to drive galactic winds (Zweibel, 2017). It is important to note that the cross section of CRs with the gas in the ISM is very small. The momentum transfer by direct interactions that lead to Gamma ray emission are negligible. The dynamical interactions are primarily transferred via the magnetic field, except for $\mathrm{CR}$ energies $\lesssim \mathrm{MeV}$. Concerning the transport and the detailed impact of CRs in the star formation process including the impact of low-energy CRs on the chemistry we refer the reader to the chapter on CRs in this volume. Here, we only give a brief outline. Being coupled to the magnetic field results in highly anisotropic transport where the particles mainly diffuse or stream along the magnetic fields (see e.g. Skilling and Strong, 1976; Cesarsky and Volk, 1978; Chandran, 2000; Padovani and Galli, 2011; Padovani et al., 2013). The lack of direct collisions also allows for CRs to move relatively freely with respect to the gas. Consequently, the energy can be redistributed independently of the local gas motions. 
With regard to the impact of CRs it is necessary to distinguish between particles with sub-relativistic energies $(\sim \mathrm{MeV})$, the GeV range, and higher energies. Low-energy CRs are an important source of heating and ionization in molecular clouds because their cross section with the thermal gas increases perceptibly (Padovani et al., 2009). They can penetrate deeply into star-forming regions and provide an effective temperature floor. In addition they directly influence chemical reaction chains and alter observables in star-forming cores (Indriolo and McCall, 2013). GeV CRs provide dynamical impact on scales of $\gtrsim 10-100 \mathrm{pc}$ by accelerating via their interaction with the magnetic field and driving galactic outflows. Energies above $\sim 10^{2}-10^{3} \mathrm{GeV}$ do not dynamically alter the star formation process.

Acknowledgements We thank the staff of the International Space Science Institute (ISSI) for their generous hospitality and for creating a stimulating collaborative environment. P.G. acknowledges funding from the European Research Council under ERC-CoG grant CRAGSMAN-646955. S.S.R.O. acknowledges funding from NSF Career grant AST-1650486. J.M.D.K. gratefully acknowledges funding from the German Research Foundation (DFG) in the form of an Emmy Noether Research Group (grant number KR4801/1-1) and a DFG Sachbeihilfe Grant (grant number KR4801/2-1), from the European Research Council (ERC) under the European Union's Horizon 2020 research and innovation programme via the ERC Starting Grant MUSTANG (grant agreement number 714907), and from Sonderforschungsbereich SFB 881 "The Milky Way System" (subproject B2) of the DFG. R.S.K. acknowledges support from the Deutsche Forschungsgemeinschaft via the SFB 881 The Milky Way System (subprojects B1, B2, and B8) as well as funding from the Heidelberg Cluster of Excellence STRUCTURES in the framework of Germanys Excellence Strategy (grant EXC-2181/1 390900948). A.G.K. acknowledges support from the NASA ATP Grant No. 80NSSC18K0561 and NASA TCAN Grant No. NNH17ZDA001N. M.P. acknowledges funding from the INAF PRIN-SKA 2017 program 1.05.01.88.04.

\section{References}

Abdo AA, Ackermann M, Ajello M, et al. (2011) Observations of the Young Supernova Remnant RX J1713.7-3946 with the Fermi Large Area Telescope. ApJ734:28

Abgrall H, Roueff E, and Drira I (2000) Total transition probability and spontaneous radiative dissociation of B, C, B' and D states of molecular hydrogen. A\&A Suppl.141:297-300

Ackermann M, Ajello M, Allafort A, et al. (2013) Detection of the Characteristic Pion-Decay Signature in Supernova Remnants. Science 339:807-811

Aharonian F, Akhperjanian AG, Aye KM, et al. (2004) Very high energy gamma rays from the direction of Sagittarius A*. A\&A425:L13-L17

Aharonian F, Akhperjanian AG, Bazer-Bachi AR, et al. (2006) The H.E.S.S. Survey of the Inner Galaxy in Very High Energy Gamma Rays. ApJ636(2):777-797

Aharonian F, Akhperjanian AG, Bazer-Bachi AR, et al. (2007) An Exceptional Very High Energy Gamma-Ray Flare of PKS 2155-304. ApJ664(2):L71-L74

Alexakis A and Biferale L (2018) Cascades and transitions in turbulent flows. Phys Reports 767:1-101 
Aluie H (2011) Compressible Turbulence: The Cascade and its Locality. PhysRevLett106(17):174502

Aluie H (2013) Scale decomposition in compressible turbulence. Physica D Nonlinear Phenomena 247(1):54-65

Aluie H, Li S, and Li H (2012) Conservative Cascade of Kinetic Energy in Compressible Turbulence. ApJ751(2):L29

Ames S (1973) Magneto-Gravitational and Thermal Instability in the Galactic Disk. ApJ182:387-404

Andrés N and Sahraoui F (2017) Alternative derivation of exact law for compressible and isothermal magnetohydrodynamics turbulence. Phys. Rev. E96(5):053205

Andrés N, Galtier S, and Sahraoui F (2018a) Exact law for homogeneous compressible Hall magnetohydrodynamics turbulence. Phys. Rev. E97(1):013204

Andrés N, Sahraoui F, Galtier S, et al. (2018b) Energy cascade rate in isothermal compressible magnetohydrodynamic turbulence. Journal of Plasma Physics 84(4):905840404

Antonia RA, Ould-Rouis M, Anselmet F, et al. (1997) Analogy between predictions of Kolmogorov and Yaglom. Journal of Fluid Mechanics 332(1):395409

Arzoumanian D, André P, Didelon P, et al. (2011) Characterizing interstellar filaments with Herschel in IC 5146. A\&A529:L6

Atkins PW and Friedman RS (2011) Molecular quantum mechanics. Oxford university press

Axford WI, Leer E, and Skadron G (1977) The acceleration of cosmic rays by shock waves. International Cosmic Ray Conference 11:132-137

Bacchini C, Fraternali F, Pezzulli G, et al. (2019) The volumetric star formation law in the Milky Way. A\&A632:A127

Bai XN, Ye J, Goodman J, et al. (2016) Magneto-thermal Disk Winds from Protoplanetary Disks. The Astrophysical Journal 818(2):152

Bakes ELO and Tielens AGGM (1994) The Photoelectric Heating Mechanism for Very Small Graphitic Grains and Polycyclic Aromatic Hydrocarbons. ApJ427:822

Balbus SA (2009) Magnetohydrodynamics of Protostellar Disks. arXiv e-prints arXiv:0906.0854

Ballesteros-Paredes J (2006) Six myths on the virial theorem for interstellar clouds. MNRAS372(1):443-449

Bally J (2011) Observations of Winds, Jets, and Turbulence Generation in GMCs. In: Alves J, Elmegreen BG, Girart JM, et al. (eds) Computational Star Formation, Proceedings of the International Astronomical Union, vol 270, pp 247-254

Bally J (2016) Protostellar Outflows. Annual Review of Astronomy and Astrophysics $54: 491-528$

Banerjee R and Pudritz RE (2006) Outflows and Jets from Collapsing Magnetized Cloud Cores. ApJ641(2):949-960

Banerjee S and Galtier S (2013) Exact relation with two-point correlation functions and phenomenological approach for compressible magnetohydro- 
dynamic turbulence. Phys. Rev. E87(1):013019

Banerjee S and Galtier S (2014) A Kolmogorov-like exact relation for compressible polytropic turbulence. Journal of Fluid Mechanics 742:230-242

Banerjee S and Galtier S (2017) An alternative formulation for exact scaling relations in hydrodynamic and magnetohydrodynamic turbulence. Journal of Physics A Mathematical General 50(1):015501

Banerjee S and Kritsuk AG (2017) Exact relations for energy transfer in selfgravitating isothermal turbulence. Phys. Rev. E96(5):053116

Banerjee S and Kritsuk AG (2018) Energy transfer in compressible magnetohydrodynamic turbulence for isothermal self-gravitating fluids. Phys. Rev. E97(2):023107

Baraffe I, Chabrier G, and Gallardo J (2009) Episodic Accretion at Early Stages of Evolution of Low-Mass Stars and Brown Dwarfs: A Solution for the Observed Luminosity Spread in H-R Diagrams? ApJ702(1):L27-L31

Batchelor GK (1953) The Theory of Homogeneous Turbulence. Cambridge University Press

Batchelor GK (1967) An Introduction to Fluid Dynamics. Cambridge University Press

Beck R (2009) Galactic and extragalactic magnetic fields - a concise review. Astrophysics and Space Sciences Transactions 5:43-47

Beck R (2012) Magnetic Fields in Galaxies. Space Science Reviews166:215-230

Beck R (2015) Magnetic fields in spiral galaxies. A\&A Review24:4

Begelman MC and McKee CF (1990) Global Effects of Thermal Conduction on Two-Phase Media. ApJ358:375

Bell AR (1978) The acceleration of cosmic rays in shock fronts. I. MNRAS182:147-156

Benzi R, Ciliberto S, Baudet C, et al. (1995) On the scaling of threedimensional homogeneous and isotropic turbulence. Physica D Nonlinear Phenomena 80(4):385-398

Beresnyak A (2019) MHD turbulence. Living Reviews in Computational Astrophysics 5:2

Beresnyak A and Lazarian A (2019) Turbulence in Magnetohydrodynamics

Bigiel F, Leroy A, Walter F, et al. (2008) The Star Formation Law in Nearby Galaxies on Sub-Kpc Scales. AJ136:2846-2871

Biskamp D (2003) Magnetohydrodynamic Turbulence

Black JH (1981) The physical state of primordial intergalactic clouds. MNRAS197:553-563

Black JH and Dalgarno A (1977) Models of interstellar clouds. I. The Zeta Ophiuchi cloud. ApJS34:405-423

Blandford RD and Ostriker JP (1978) Particle acceleration by astrophysical shocks. ApJ221:L29-L32

Blandford RD and Payne DG (1982) Hydromagnetic flows from accretion disks and the production of radio jets. Monthly Notices of the Royal Astronomical Society 199:883-903

Boldyrev S, Nordlund A, and Padoan P (2002) Scaling Relations of Supersonic Turbulence in Star-forming Molecular Clouds. ApJ573(2):678-684 
Bonnell IA, Bate MR, Clarke CJ, et al. (2001) Competitive accretion in embedded stellar clusters. MNRAS323(4):785-794

Bonnor WB (1956) Boyle's Law and gravitational instability. MNRAS116:351

Bourke TL, Myers PC, Robinson G, et al. (2001) New OH Zeeman Measurements of Magnetic Field Strengths in Molecular Clouds. ApJ554(2):916-932

Bouvier J, Matt SP, Mohanty S, et al. (2014) Angular Momentum Evolution of Young Low-Mass Stars and Brown Dwarfs: Observations and Theory. Protostars and Planets VI pp 433-450

Brandenburg A (2018) Advances in mean-field dynamo theory and applications to astrophysical turbulence. Journal of Plasma Physics 84(4):735840404

Brandenburg A, Sokoloff D, and Subramanian K (2012) Current Status of Turbulent Dynamo Theory. From Large-Scale to Small-Scale Dynamos. Space Science Reviews169(1-4):123-157

Bressert E, Bastian N, Gutermuth R, et al. (2010) The spatial distribution of star formation in the solar neighbourhood: do all stars form in dense clusters? MNRAS409:L54-L58

Bron E, Le Bourlot J, and Le Petit F (2014) Surface chemistry in the interstellar medium. II. $\mathrm{H}_{2}$ formation on dust with random temperature fluctuations. A\&A569:A100

Brunt CM and Federrath C (2014) An observational method to measure the relative fractions of solenoidal and compressible modes in interstellar clouds. MNRAS442(2):1451-1469

Brunt CM, Heyer MH, and Mac Low MM (2009) Turbulent driving scales in molecular clouds. A\&A504(3):883-890

Brunt CM, Federrath C, and Price DJ (2010) A method for reconstructing the variance of a 3D physical field from 2D observations: application to turbulence in the interstellar medium. MNRAS403(3):1507-1515

Burton MG, Hollenbach DJ, and Tielens AGGM (1990) Line Emission from Clumpy Photodissociation Regions. ApJ365:620

Caprioli D and Spitkovsky A (2014) Simulations of Ion Acceleration at Nonrelativistic Shocks. I. Acceleration Efficiency. ApJ783(2):91

Carroll JJ, Frank A, Blackman EG, et al. (2009) Outflow-Driven Turbulence in Molecular Clouds. The Astrophysical Journal 695(2):1376-1381

Caselli P, Walmsley CM, Terzieva R, et al. (1998) The Ionization Fraction in Dense Cloud Cores. ApJ499(1):234-249

Cen R (1992) A Hydrodynamic Approach to Cosmology: Methodology. ApJS78:341

Cen R and Fang T (2006) Where Are the Baryons? III. Nonequilibrium Effects and Observables. ApJ650(2):573-591

Cesarsky CJ and Volk HJ (1978) Cosmic Ray Penetration into Molecular Clouds. A\&A70:367

Ceverino D, Dekel A, and Bournaud F (2010) High-redshift clumpy discs and bulges in cosmological simulations. MNRAS404(4):2151-2169

Chabrier G (2003) Galactic Stellar and Substellar Initial Mass Function. PASP115:763-795 
Chandran BDG (2000) Confinement and Isotropization of Galactic Cosmic Rays by Molecular-Cloud Magnetic Mirrors When Turbulent Scattering Is Weak. ApJ529(1):513-535

Chandrasekhar S (1951) The fluctuations of density in isotropic turbulence. Proceedings of the Royal Society of London Series A, Mathematical and Physical Sciences 210(1100):18-25, http://www.jstor.org/stable/98946

Chassaing P (1985) An alternative formulation of the equations of turbulent motion for a fluid of variable density. Journal de Mecanique Theorique et Appliquee 4(3):375-389

Chassaing P, Antonia R, Anselmet F, et al. (2002) Variable Density Fluid Turbulence, Fluid Mechanics and its Applications, vol 69. Kluwer Academic Ppublishers

Chen S, Xia Z, Wang J, et al. (2015) Recent progress in compressible turbulence. Acta Mechanica Sinica 31(3):275

Chevance M, Kruijssen JMD, Hygate APS, et al. (2019) The lifecycle of molecular clouds in nearby star-forming disc galaxies. MNRAS in press $\mathrm{p}$ arXiv:1911.03479

Chiuderi C and Velli M (2015) Basics of Plasma Astrophysics

Choi E and Stone JM (2012) The Effect of Anisotropic Conduction on the Thermal Instability in the Interstellar Medium. ApJ747:86

Chu BT and Kovasznay LSG (1957) Non-linear interactions in a viscous heatconducting compressible gas. Journal of Fluid Mechanics 3:494-514

Churazov E, Sunyaev R, Isern J, et al. (2014) Cobalt-56 $\gamma$-ray emission lines from the type Ia supernova 2014J. Nature512(7515):406-408

Churchwell E, Povich MS, Allen D, et al. (2006) The Bubbling Galactic Disk. ApJ649(2):759-778

Clark PC and Glover SCO (2014) On column density thresholds and the star formation rate. MNRAS444(3):2396-2414

Clark PC, Glover SCO, Klessen RS, et al. (2011) Gravitational Fragmentation in Turbulent Primordial Gas and the Initial Mass Function of Population III Stars. ApJ727(2):110

Clark PC, Glover SCO, Ragan SE, et al. (2013) On the Temperature Structure of the Galactic Center Cloud G0.253+0.016. ApJ768(2):L34

Commerçon B, Hennebelle P, and Henning T (2011) Collapse of Massive Magnetized Dense Cores Using Radiation Magnetohydrodynamics: Early Fragmentation Inhibition. ApJ742:L9

Commerçon B, Hennebelle P, Audit E, et al. (2010) Protostellar collapse: radiative and magnetic feedbacks on small-scale fragmentation. A\&A510:L3

Congiu E, Matar E, Kristensen LE, et al. (2009) Laboratory evidence for the non-detection of excited nascent $\mathrm{H}_{2}$ in dark clouds. MNRAS397(1):L96 L100

Cook AW and Zhou Y (2002) Energy transfer in rayleigh-taylor instability. Phys Rev E 66:026312, https://link.aps.org/doi/10.1103/PhysRevE.66.026312

Crutcher RM (1999) Magnetic Fields in Molecular Clouds: Observations Confront Theory. ApJ520(2):706-713 
Crutcher RM (2012) Magnetic Fields in Molecular Clouds. ARA\&A50:29-63

Crutcher RM, Wandelt B, Heiles C, et al. (2010) Magnetic Fields in Interstellar Clouds from Zeeman Observations: Inference of Total Field Strengths by Bayesian Analysis. ApJ725:466-479

Cyganowski CJ, Whitney BA, Holden E, et al. (2008) A Catalog of Extended Green Objects in the GLIMPSE Survey: A New Sample of Massive Young Stellar Object Outflow Candidates. The Astronomical Journal 136(6):23912412

Daddi E, Elbaz D, Walter F, et al. (2010) Different Star Formation Laws for Disks Versus Starbursts at Low and High Redshifts. ApJ714:L118-L122

Dale JE, Ercolano B, and Bonnell IA (2013) Ionizing feedback from massive stars in massive clusters - III. Disruption of partially unbound clouds. MNRAS430(1):234-246

Dalgarno A and Black JH (1976) REVIEW: Molecule formation in the interstellar gas. Reports on Progress in Physics 39(6):573-612

Dalgarno A, Yan M, and Liu W (1999) Electron Energy Deposition in a Gas Mixture of Atomic and Molecular Hydrogen and Helium. ApJS125(1):237256

Davidson PA (2004) Turbulence : an introduction for scientists and engineers

Davis J Leverett and Greenstein JL (1951) The Polarization of Starlight by Aligned Dust Grains. ApJ114:206

de Avillez MA and Breitschwerdt D (2012) The Diagnostic O VI Absorption Line in Diffuse Plasmas: Comparison of Non-equilibrium Ionization Structure Simulations to FUSE Data. ApJ761(2):L19

Diamond PH, Itoh SI, and Itoh K (2010) Modern Plasma Physics

Dib S, Kim J, Vázquez-Semadeni E, et al. (2007) The Virial Balance of Clumps and Cores in Molecular Clouds. ApJ661(1):262-284

Diehl R, Siegert T, Hillebrandt W, et al. (2014) Early ${ }^{56} \mathrm{Ni}$ decay gamma rays from SN2014J suggest an unusual explosion. Science 345:1162-1165

Donzis DA and Jagannathan S (2013) Fluctuations of thermodynamic variables in stationary compressible turbulence. Journal of Fluid Mechanics 733:221-244

Donzis DA and Panickacheril J (2019) Universality and scaling in compressible turbulence. arXiv e-prints arXiv:1907.07871

Draine BT (2011) Physics of the Interstellar and Intergalactic Medium

Draine BT and Bertoldi F (1996) Structure of Stationary Photodissociation Fronts. ApJ468:269

Draine BT and Lee HM (1984) Optical Properties of Interstellar Graphite and Silicate Grains. ApJ285:89

Draine BT and Li A (2007) Infrared Emission from Interstellar Dust. IV. The Silicate-Graphite-PAH Model in the Post-Spitzer Era. ApJ657(2):810-837

Draine BT and Sutin B (1987) Collisional Charging of Interstellar Grains. ApJ320:803

Draine BT, Roberge WG, and Dalgarno A (1983) Magnetohydrodynamic shock waves in molecular clouds. ApJ264:485-507 
Dutrey A, Duvert G, Castets A, et al. (1991) Periodically spaced fragmentation in Orion A. A\&A247:L9

Ebert R (1955) Über die Verdichtung von H I-Gebieten. Mit 5 Textabbildungen. Zeitschrift für Astrophysik37:217-+

Ebinger K, Curtis S, Fröhlich C, et al. (2019) PUSHing Core-collapse Supernovae to Explosions in Spherical Symmetry. II. Explodability and Remnant Properties. ApJ870(1):1

Elmegreen BG and Burkert A (2010) Accretion-Driven Turbulence and the Transition to Global Instability in Young Galaxy Disks. ApJ712(1):294-302

Elmegreen BG and Scalo J (2004) Interstellar Turbulence I: Observations and Processes. ARA\&A42:211-273

Elphick C, Regev O, and Shaviv N (1992) Dynamics of Fronts in Thermally Bistable Fluids. ApJ392:106

Eyink GL and Drivas TD (2018) Cascades and Dissipative Anomalies in Compressible Fluid Turbulence. Physical Review X 8(1):011022

Eyink GL and Sreenivasan KR (2006) Onsager and the theory of hydrodynamic turbulence. Reviews of Modern Physics 78(1):87-135

Falgarone E, Pineau des Forets G, and Roueff E (1995) Chemical signatures of the intermittency of turbulence in low density interstellar clouds. A\&A300:870

Falkovich G and Kritsuk AG (2017) How vortices and shocks provide for a flux loop in two-dimensional compressible turbulence. Physical Review Fluids 2(9):092603

Falkovich G, Fouxon I, and Oz Y (2010) New relations for correlation functions in Navier-Stokes turbulence. Journal of Fluid Mechanics 644:465

Faucher-Giguère CA, Quataert E, and Hopkins PF (2013) Feedback-regulated star formation in molecular clouds and galactic discs. MNRAS433(3):1970 1990

Favre A (1983) Turbulence: Space-time statistical properties and behavior in supersonic flows. Physics of Fluids 26(10):2851-2863

Federrath C (2018) The turbulent formation of stars. Physics Today 71(6):3842

Federrath C, Schrön M, Banerjee R, et al. (2014) Modeling Jet and Outflow Feedback during Star Cluster Formation. The Astrophysical Journal $790(2): 128$

Federrath C, Klessen RS, Iapichino L, et al. (2016) The world's largest turbulence simulations. arXiv e-prints arXiv:1607.00630

Feireisl E (2004) Dynamics of Viscous Compressible Fluids. Oxford University Press

Feireisl E, Karper TG, and Pokorný M (2016) Mathematical Theory of Compressible Viscous Fluids. Lecture Notes in Mathematical Fluid Mechanics, Birkhäuser Basel

Feldmann R, Gnedin NY, and Kravtsov AV (2011) How Universal is the Sigma_SFR \{-\}Sigma_H_2 Relation? ApJ732(2):115

Ferrière KM (2001) The interstellar environment of our galaxy. Reviews of Modern Physics 73:1031-1066 
Fiege JD and Pudritz RE (2000) Helical fields and filamentary molecular clouds - I. MNRAS311(1):85-104

Field GB (1965) Thermal Instability. ApJ142:531

Field GB, Goldsmith DW, and Habing HJ (1969) Cosmic-Ray Heating of the Interstellar Gas. ApJ155:L149

Fleck J Robert C (1996) Scaling Relations for the Turbulent, Non-Selfgravitating, Neutral Component of the Interstellar Medium. ApJ458:739

Fletcher A, Beck R, Shukurov A, et al. (2011) Magnetic fields and spiral arms in the galaxy M51. MNRAS412:2396-2416

Flower DR, Le Bourlot J, Pineau des Forêts G, et al. (2003) The contributions of J-type shocks to the $\mathrm{H}_{2}$ emission from molecular outflow sources. MNRAS341(1):70-80

Foster PN and Chevalier RA (1993) Gravitational Collapse of an Isothermal Sphere. ApJ416:303

Frisch U (1995) Turbulence

Fukui Y, Mizuno N, Yamaguchi R, et al. (2001) On the Mass Spectrum of Giant Molecular Clouds in the Large Magellanic Cloud. PASJ53(6):L41L44

Gaensler BM, Madsen GJ, Chatterjee S, et al. (2008) The Vertical Structure of Warm Ionised Gas in the Milky Way. PASA25(4):184-200

Galametz M, Madden SC, Galliano F, et al. (2011) Probing the dust properties of galaxies up to submillimetre wavelengths. II. Dust-to-gas mass ratio trends with metallicity and the submm excess in dwarf galaxies. A\&A532:A56

Galtier S (2018) Turbulence in space plasmas and beyond. Journal of Physics A: Mathematical and Theoretical 51(29):293001

Galtier S and Banerjee S (2011) Exact Relation for Correlation Functions in Compressible Isothermal Turbulence. PhysRevLett107(13):134501

Geen S, Rosdahl J, Blaizot J, et al. (2015) A detailed study of feedback from a massive star. MNRAS448:3248-3264

Genzel R, Tacconi LJ, Gracia-Carpio J, et al. (2010) A study of the gas-star formation relation over cosmic time. MNRAS407:2091-2108

Girichidis P, Federrath C, Banerjee R, et al. (2011) Importance of the initial conditions for star formation - I. Cloud evolution and morphology. MNRAS413:2741-2759

Girichidis P, Federrath C, Banerjee R, et al. (2012) Importance of the initial conditions for star formation - II. Fragmentation-induced starvation and accretion shielding. MNRAS420:613-626

Girichidis P, Konstandin L, Whitworth AP, et al. (2014) On the Evolution of the Density Probability Density Function in Strongly Self-gravitating Systems. ApJ781:91

Girichidis P, Walch S, Naab T, et al. (2016) The SILCC (SImulating the LifeCycle of molecular Clouds) project - II. Dynamical evolution of the supernova-driven ISM and the launching of outflows. MNRAS456:3432-3455

Girichidis P, Seifried D, Naab T, et al. (2018) The SILCC project - V. The impact of magnetic fields on the chemistry and the formation of molecular 
clouds. MNRAS480:3511-3540

Glassgold AE and Langer WD (1975) The $\mathrm{C}^{+}-\mathrm{CO}$ transition in interstellar clouds. ApJ197:347-350

Glassgold AE, Galli D, and Padovani M (2012) Cosmic-Ray and X-Ray Heating of Interstellar Clouds and Protoplanetary Disks. ApJ756(2):157

Glover SCO (2003) Comparing Gas-Phase and Grain-catalyzed $\mathrm{H}_{2}$ Formation. ApJ584(1):331-338

Glover SCO and Clark PC (2012a) Approximations for modelling CO chemistry in giant molecular clouds: a comparison of approaches. MNRAS421:116-131

Glover SCO and Clark PC (2012b) Is molecular gas necessary for star formation? MNRAS421:9-19

Glover SCO and Clark PC (2012c) Star formation in metal-poor gas clouds. MNRAS426(1):377-388

Glover SCO and Jappsen AK (2007) Star Formation at Very Low Metallicity. I. Chemistry and Cooling at Low Densities. ApJ666(1):1-19

Glover SCO and Mac Low MM (2007) Simulating the Formation of Molecular Clouds. II. Rapid Formation from Turbulent Initial Conditions. ApJ659(2):1317-1337

Gnat O and Ferland GJ (2012) Ion-by-ion Cooling Efficiencies. ApJS199:20

Gnedin NY and Hollon N (2012) Cooling and Heating Functions of Photoionized Gas. ApJS202(2):13

Godard B, Falgarone E, and Pineau Des Forêts G (2009) Models of turbulent dissipation regions in the diffuse interstellar medium. A\&A495(3):847-867

Goldreich P and Kwan J (1974) Molecular Clouds. ApJ189:441-454

Goldsmith PF (2001) Molecular Depletion and Thermal Balance in Dark Cloud Cores. ApJ557:736-746

Goldsmith PF and Langer WD (1978) Molecular cooling and thermal balance of dense interstellar clouds. ApJ222:881-895

Gong M, Ostriker EC, and Wolfire MG (2017) A Simple and Accurate Network for Hydrogen and Carbon Chemistry in the Interstellar Medium. ApJ843:38

Gould RJ and Salpeter EE (1963) The Interstellar Abundance of the Hydrogen Molecule. I. Basic Processes. ApJ138:393

Graham JP, Cameron R, and Schssler M (2010) TURBULENT SMALLSCALE DYNAMO ACTION IN SOLAR SURFACE SIMULATIONS. The Astrophysical Journal 714(2):1606-1616

Gredel R, Lepp S, and Dalgarno A (1987) The C/CO Ratio in Dense Interstellar Clouds. ApJ323:L137

Gredel R, Lepp S, Dalgarno A, et al. (1989) Cosmic-Ray-induced Photodissociation and Photoionization Rates of Interstellar Molecules. ApJ347:289

Greif TH, Springel V, White SDM, et al. (2011) Simulations on a Moving Mesh: The Clustered Formation of Population III Protostars. ApJ737(2):75

Grenier IA, Black JH, and Strong AW (2015) The Nine Lives of Cosmic Rays in Galaxies. ARA\&A53:199-246

Grete P, O'Shea BW, Beckwith K, et al. (2017) Energy transfer in compressible magnetohydrodynamic turbulence. Physics of Plasmas 24(9):092311 
Gutermuth RA, Pipher JL, Megeath ST, et al. (2011) A Correlation between Surface Densities of Young Stellar Objects and Gas in Eight Nearby Molecular Clouds. ApJ739:84

Habing HJ (1968) The interstellar radiation density between 912 A and 2400 A. BAN19:421

Haffner LM, Dettmar RJ, Beckman JE, et al. (2009) The warm ionized medium in spiral galaxies. Reviews of Modern Physics 81(3):969-997

Hall JS (1949) Observations of the Polarized Light from Stars. Science 109(2825):166-167

Hansen CE, Klein RI, McKee CF, et al. (2012) Feedback Effects on Low-mass Star Formation. The Astrophysical Journal 747(1):22

Hansen CJ, Kawaler SD, and Trimble V (2004) Stellar interiors : physical principles, structure, and evolution

Hartmann L, Herczeg G, and Calvet N (2016) Accretion onto Pre-MainSequence Stars. ARA\&A54:135-180

Haverkorn M (2015) Magnetic Fields in the Milky Way. In: Lazarian A, de Gouveia Dal Pino EM, and Melioli C (eds) Magnetic Fields in Diffuse Media, Astrophysics and Space Science Library, vol 407, p 483

Hayward CC and Hopkins PF (2017) How stellar feedback simultaneously regulates star formation and drives outflows. MNRAS465(2):1682-1698

Heesen V, Brinks E, Krause MGH, et al. (2015) The non-thermal superbubble in IC 10: the generation of cosmic ray electrons caught in the act. MNRAS447:L1-L5

Heiles C and Crutcher R (2005) Magnetic Fields in Diffuse HI and Molecular Clouds. In: Wielebinski R and Beck R (eds) Cosmic Magnetic Fields, Lecture Notes in Physics, Berlin Springer Verlag, vol 664, p 137

Helmholtz H (1858) ber integrale der hydrodynamischen gleichungen, welche den wirbelbewegungen entsprechen. Journal fr die reine und angewandte Mathematik 55:25-55, http://eudml.org/doc/147720

Hennebelle P and Falgarone E (2012) Turbulent molecular clouds. A\&A Review20:55

Hennebelle P and Fromang S (2008) Magnetic processes in a collapsing dense core. I. Accretion and ejection. A\&A477(1):9-24

Hennebelle P and Inutsuka Si (2019) The role of magnetic field in molecular cloud formation and evolution. Frontiers in Astronomy and Space Sciences $6: 5$

Hennebelle P and Pérault M (1999) Dynamical condensation in a thermally bistable flow. Application to interstellar cirrus. A\&A351:309-322

Hennebelle P, Whitworth AP, Gladwin PP, et al. (2003) Protostellar collapse induced by compression. MNRAS340(3):870-882

Henriksen RN (1991) On Molecular Cloud Scaling Laws and Star Formation. ApJ377:500

Herrera-Camus R, Fisher DB, Bolatto AD, et al. (2012) Dust-to-gas Ratio in the Extremely Metal-poor Galaxy I Zw 18. ApJ752(2):112

Heyer M and Dame TM (2015) Molecular Clouds in the Milky Way. ARA\&A53:583-629 
Heyer M, Krawczyk C, Duval J, et al. (2009) Re-Examining Larson's Scaling Relationships in Galactic Molecular Clouds. ApJ699:1092-1103

Heyer MH, Brunt C, Snell RL, et al. (1998) The Five College Radio Astronomy Observatory CO Survey of the Outer Galaxy. ApJS115(2):241-258

Hill AS, Joung MR, Mac Low MM, et al. (2012) Vertical Structure of a Supernova-driven Turbulent, Magnetized Interstellar Medium. ApJ750:104

Hiltner WA (1949) On the Presence of Polarization in the Continuous Radiation of Stars. II. ApJ109:471

Hirano S, Hosokawa T, Yoshida N, et al. (2014) One Hundred First Stars: Protostellar Evolution and the Final Masses. ApJ781(2):60

Hocuk S and Spaans M (2010) The impact of X-rays on molecular cloud fragmentation and the inital mass function. A\&A522:A24

Hollenbach D and McKee CF (1989) Molecule Formation and Infrared Emission in Fast Interstellar Shocks. III. Results for J Shocks in Molecular Clouds. ApJ342:306

Hopkins PF, Kereš D, Oñorbe J, et al. (2014) Galaxies on FIRE (Feedback In Realistic Environments): stellar feedback explains cosmologically inefficient star formation. MNRAS445:581-603

Hopkins PF, Wetzel A, Kereš D, et al. (2018) FIRE-2 simulations: physics versus numerics in galaxy formation. MNRAS480(1):800-863

Hosokawa T, Offner SSR, and Krumholz MR (2011) On the Reliability of Stellar Ages and Age Spreads Inferred from Pre-main-sequence Evolutionary Models. ApJ738(2):140

Hoyle F (1953) On the Fragmentation of Gas Clouds Into Galaxies and Stars. ApJ118:513

Hoyle F and Ellis GRA (1963) On the Existence of an Ionized Layer about the Galactic Plane. Australian Journal of Physics 16:1

Indriolo N and McCall BJ (2013) Cosmic-ray astrochemistry. Chemical Society Reviews 42:7763-7773

Inoue T, Inutsuka Si, and Koyama H (2006) Structure and Stability of Phase Transition Layers in the Interstellar Medium. ApJ652(2):1331-1338

Inoue T, Inutsuka Si, and Koyama H (2007) The Role of Ambipolar Diffusion in the Formation Process of Moderately Magnetized Diffuse Clouds. ApJ658:L99-L102

Jagannathan S and Donzis DA (2016) Reynolds and Mach number scaling in solenoidally-forced compressible turbulence using high-resolution direct numerical simulations. Journal of Fluid Mechanics 789:669-707

Jeans JH (1902) The Stability of a Spherical Nebula. Royal Society of London Philosophical Transactions Series A 199:1-53

Jeffreson SMR and Kruijssen JMD (2018) A general theory for the lifetimes of giant molecular clouds under the influence of galactic dynamics. MNRAS476:3688-3715

Jenkins EB (2013) The Fractional Ionization of the Warm Neutral Interstellar Medium. ApJ764(1):25

Jha SW, Maguire K, and Sullivan M (2019) Observational properties of thermonuclear supernovae. Nature Astronomy 3:706-716 
Joung MR, Mac Low MM, and Bryan GL (2009) Dependence of Interstellar Turbulent Pressure on Supernova Rate. ApJ704(1):137-149

Jura M (1975) Interstellar clouds containing optically thin $\mathrm{H}_{2}$. ApJ197:575580

Kainulainen J, Beuther H, Henning T, et al. (2009) Probing the evolution of molecular cloud structure. From quiescence to birth. A\&A508:L35-L38

Kalberla PMW and Kerp J (2009) The Hi Distribution of the Milky Way. ARA\&A47(1):27-61

de Karman T and Howarth L (1938) On the statistical theory of isotropic turbulence. Proc Roy Soc London A 164:192-215

Kennicutt RC and Evans NJ (2012) Star Formation in the Milky Way and Nearby Galaxies. ARA\&A50:531-608

Kennicutt RC Jr (1989) The star formation law in galactic disks. ApJ344:685703

Kennicutt RC Jr (1998) The Global Schmidt Law in Star-forming Galaxies. ApJ498:541-552

Kennicutt RC Jr, Calzetti D, Walter F, et al. (2007) Star Formation in NGC 5194 (M51a). II. The Spatially Resolved Star Formation Law. ApJ671:333348

Khintchine A (1934) Korrelationstheorie der stationären stochastischen prozesse. Mathematische Annalen 109:604-615, http://eudml.org/doc/159698

Kida S and Orszag SA (1990) Energy and spectral dynamics in forced compressible turbulence. Journal of Scientific Computing 5:85-125

Kida S and Orszag SA (1992) Energy and spectral dynamics in decaying compressible turbulence. Journal of Scientific Computing 7:1-34

Kim CG and Ostriker EC (2015) Momentum Injection by Supernovae in the Interstellar Medium. ApJ802:99

Kim J and Ryu D (2005) Density Power Spectrum of Compressible Hydrodynamic Turbulent Flows. ApJ630(1):L45-L48

Kim JG and Kim WT (2013) Instability of Evaporation Fronts in the Interstellar Medium. ApJ779(1):48

Klessen RS and Burkert A (2001) The Formation of Stellar Clusters: Gaussian Cloud Conditions. II. ApJ549:386-401

Klessen RS and Glover SCO (2016) Physical Processes in the Interstellar Medium. Saas-Fee Advanced Course 43:85

Klessen RS and Hennebelle P (2010) Accretion-driven turbulence as universal process: galaxies, molecular clouds, and protostellar disks. A\&A520:A17

Kolmogorov A (1941a) The Local Structure of Turbulence in Incompressible Viscous Fluid for Very Large Reynolds Numbers. Akademiia Nauk SSSR Doklady 30:301-305

Kolmogorov AN (1941b) Dissipation of Energy in Locally Isotropic Turbulence. Akademiia Nauk SSSR Doklady 32:16

Kovásznay LSG (1953) Turbulence in supersonic flow. Journal of the Aeronautical Sciences 20(10):657-674, https://doi.org/10.2514/8.2793 
Kowal G and Lazarian A (2007) Scaling Relations of Compressible MHD Turbulence. ApJ666(2):L69-L72

Koyama H and Inutsuka SI (2000) Molecular Cloud Formation in Shockcompressed Layers. ApJ532:980-993

Kraft RP (1967) Studies of Stellar Rotation. V. The Dependence of Rotation on Age among Solar-Type Stars. ApJ150:551

Kraichnan RH (1955) On the Statistical Mechanics of an Adiabatically Compressible Fluid. Acoustical Society of America Journal 27(3):438

Kreckel K, Faesi C, Kruijssen JMD, et al. (2018) A 50 pc Scale View of Star Formation Efficiency across NGC 628. ApJ863:L21

Kritsuk AG (2019) Energy Transfer and Spectra in Simulations of Twodimensional Compressible Turbulence, ERCOFTAC Series, vol 26, Springer Nature Switzerland AG, pp 61-70

Kritsuk AG and Banerjee S (2020) Energy Transfer in Subsonic Isothermal Turbulence. Phys Rev Fluids In preparation

Kritsuk AG and Norman ML (2002) Interstellar Phase Transitions Stimulated by Time-dependent Heating. ApJ580(1):L51-L55

Kritsuk AG, Norman ML, and Padoan P (2006a) Adaptive Mesh Refinement for Supersonic Molecular Cloud Turbulence. ApJ638(1):L25-L28

Kritsuk AG, Wagner R, Norman ML, et al. (2006b) High resolution simulations of supersonic turbulence in molecular clouds, Astronomical Society of the Pacific Conference Series, vol 359, p 84

Kritsuk AG, Norman ML, Padoan P, et al. (2007a) The Statistics of Supersonic Isothermal Turbulence. ApJ665(1):416-431

Kritsuk AG, Padoan P, Wagner R, et al. (2007b) Scaling laws and intermittency in highly compressible turbulence. In: Shaikh D and Zank GP (eds) Turbulence and Nonlinear Processes in Astrophysical Plasmas, American Institute of Physics Conference Series, vol 932, pp 393-399

Kritsuk AG, Ustyugov SD, Norman ML, et al. (2009) Simulating supersonic turbulence in magnetized molecular clouds. In: Journal of Physics Conference Series, Journal of Physics Conference Series, vol 180, p 012020

Kritsuk AG, Ustyugov SD, Norman ML, et al. (2010) Self-organization in Turbulent Molecular Clouds: Compressional Versus Solenoidal Modes, Astronomical Society of the Pacific Conference Series, vol 429, p 15

Kritsuk AG, Lee CT, and Norman ML (2013a) A supersonic turbulence origin of Larson's laws. MNRAS436(4):3247-3261

Kritsuk AG, Wagner R, and Norman ML (2013b) Energy cascade and scaling in supersonic isothermal turbulence. Journal of Fluid Mechanics 729:R1

Kritsuk AG, Wagner R, and Norman ML (2015) Scaling in Supersonic Isothermal Turbulence, Astronomical Society of the Pacific Conference Series, vol 498, p 16

Kritsuk AG, Ustyugov SD, and Norman ML (2017) The structure and statistics of interstellar turbulence. New Journal of Physics 19(6):065003

Kroupa P (2001) On the variation of the initial mass function. MNRAS322:231-246 
Kruijssen JMD (2012) On the fraction of star formation occurring in bound stellar clusters. MNRAS426:3008-3040

Kruijssen JMD and Longmore SN (2014) An uncertainty principle for star formation - I. Why galactic star formation relations break down below a certain spatial scale. MNRAS439:3239-3252

Kruijssen JMD, Maschberger T, Moeckel N, et al. (2012) The dynamical state of stellar structure in star-forming regions. MNRAS419:841-853

Kruijssen JMD, Schruba A, Hygate APS, et al. (2018) An uncertainty principle for star formation - II. A new method for characterizing the cloud-scale physics of star formation and feedback across cosmic history. MNRAS479:1866-1952

Kruijssen JMD, Schruba A, Chevance M, et al. (2019) Fast and inefficient star formation due to short-lived molecular clouds and rapid feedback. Nature569(7757):519-522

Krumholz MR and Federrath C (2019) The Role of Magnetic Fields in Setting the Star Formation Rate and the Initial Mass Function. Frontiers in Astronomy and Space Sciences 6:7

Krumholz MR, McKee CF, and Klein RI (2005) How Protostellar Outflows Help Massive Stars Form. ApJ618(1):L33-L36

Krumholz MR, Klein RI, McKee CF, et al. (2009a) The Formation of Massive Star Systems by Accretion. Science 323(5915):754

Krumholz MR, McKee CF, and Tumlinson J (2009b) The Atomic-to-Molecular Transition in Galaxies. II: H I and $\mathrm{H}_{2}$ Column Densities. ApJ693:216-235

Krumholz MR, McKee CF, and Tumlinson J (2009c) The Star Formation Law in Atomic and Molecular Gas. ApJ699:850-856

Krumholz MR, Leroy AK, and McKee CF (2011) Which Phase of the Interstellar Medium Correlates with the Star Formation Rate? ApJ731(1):25

Krumholz MR, Dekel A, and McKee CF (2012) A Universal, Local Star Formation Law in Galactic Clouds, nearby Galaxies, High-redshift Disks, and Starbursts. ApJ745:69

Krumholz MR, Burkhart B, Forbes JC, et al. (2018) A unified model for galactic discs: star formation, turbulence driving, and mass transport. MNRAS477(2):2716-2740

Krumholz MR, McKee CF, and Bland -Hawthorn J (2019) Star Clusters Across Cosmic Time. ARA\&A57:227-303

Krymskii GF (1977) A regular mechanism for the acceleration of charged particles on the front of a shock wave. Akademiia Nauk SSSR Doklady 234:13061308

Kulsrud R and Pearce WP (1969) The Effect of Wave-Particle Interactions on the Propagation of Cosmic Rays. ApJ156:445

Lada CJ and Lada EA (2003) Embedded Clusters in Molecular Clouds. ARA\&A41:57-115

Lada CJ, Lombardi M, Roman-Zuniga C, et al. (2013) Schmidt's Conjecture and Star Formation in Molecular Clouds. ApJ778(2):133

Lamers HJGLM and Cassinelli JP (1999) Introduction to Stellar Winds

Landau LD and Lifshitz EM (1987) Fluid Mechanics 
Langer W (1976) The carbon monoxide abundance in interstellar clouds. ApJ206:699-712

Larson RB (1969) Numerical calculations of the dynamics of collapsing protostar. MNRAS145:271

Larson RB (1981) Turbulence and star formation in molecular clouds. MNRAS194:809-826

Larson RB (1985) Cloud fragmentation and stellar masses. MNRAS214:379 398

Latter WB and Black JH (1991) Molecular Hydrogen Formation by Excited Atom Radiative Association. ApJ372:161

Lazarian A, Eyink GL, Jafari A, et al. (2020) 3d turbulent reconnection: Theory, tests, and astrophysical implications. Physics of Plasmas 27(1):012305, https://doi.org/10.1063/1.5110603

Le Bourlot J, Le Petit F, Pinto C, et al. (2012) Surface chemistry in the interstellar medium. I. $\mathrm{H}_{2}$ formation by Langmuir-Hinshelwood and EleyRideal mechanisms. A\&A541:A76

Lee CF, Hirano N, Palau A, et al. (2009) Rotation and Outflow Motions in the Very Low-Mass Class 0 Protostellar System HH 211 at Subarcsecond Resolution. The Astrophysical Journal 699(2):1584-1594

Lees A and Aluie H (2019) Baropycnal work: A mechanism for energy transfer across scales. Fluids 4(2):92, http://dx.doi.org/10.3390/fluids4020092

Lele SK (1994) Compressibility effects on turbulence. Annual Review of Fluid Mechanics 26:211-254

Lequeux J (2005) The Interstellar Medium

Leroy AK, Walter F, Sandstrom K, et al. (2013) Molecular Gas and Star Formation in nearby Disk Galaxies. AJ146:19

Leung CM (1975) Radiation transport in dense interstellar dust clouds. ApJ199:340-360

Li PS, Myers A, and McKee CF (2012) Ambipolar Diffusion Heating in Turbulent Systems. ApJ760(1):33

Lighthill MJ (1955) The Effect of Compressibility on Turbulence. In: Gas Dynamics of Cosmic Clouds, IAU Symposium, vol 2, p 121

Liu G, Koda J, Calzetti D, et al. (2011) The Super-linear Slope of the Spatially Resolved Star Formation Law in NGC 3521 and NGC 5194 (M51a). ApJ735:63

Longmore SN, Kruijssen JMD, Bastian N, et al. (2014) The Formation and Early Evolution of Young Massive Clusters. Protostars and Planets VI pp 291-314

Lu X, Zhang Q, Liu HB, et al. (2018) Filamentary Fragmentation and Accretion in High-mass Star-forming Molecular Clouds. ApJ855(1):9

Lucy LB and Solomon PM (1970) Mass Loss by Hot Stars. ApJ159:879

Mac Low MM (1999) The Energy Dissipation Rate of Supersonic, Magnetohydrodynamic Turbulence in Molecular Clouds. ApJ524(1):169-178

Mac Low MM and Klessen RS (2004) Control of star formation by supersonic turbulence. Reviews of Modern Physics 76:125-194 
Mac Low MM, Klessen RS, Burkert A, et al. (1998) Kinetic Energy Decay Rates of Supersonic and Super-Alfvénic Turbulence in Star-Forming Clouds. PhysRevLett80(13):2754-2757

Machida MN and Hosokawa T (2013) Evolution of protostellar outflow around low-mass protostar. MNRAS431(2):1719-1744

Machida MN, Inutsuka Si, and Matsumoto T (2008) High- and Low-Velocity Magnetized Outflows in the Star Formation Process in a Gravitationally Collapsing Cloud. ApJ676(2):1088-1108

Madore BF (1977) Numerical simulations of the rate of star formation in external galaxies. MNRAS178:1-9

Maio U, Dolag K, Ciardi B, et al. (2007) Metal and molecule cooling in simulations of structure formation. MNRAS379(3):963-973

Marcowith A, Bret A, Bykov A, et al. (2016) The microphysics of collisionless shock waves. Reports on Progress in Physics 79(4):046901

Masunaga H and Inutsuka Si (2000) A Radiation Hydrodynamic Model for Protostellar Collapse. II. The Second Collapse and the Birth of a Protostar. ApJ531(1):350-365

Mathis JS, Rumpl W, and Nordsieck KH (1977) The size distribution of interstellar grains. ApJ217:425-433

Mazzali PA, McFadyen AI, Woosley SE, et al. (2014) An upper limit to the energy of gamma-ray bursts indicates that GRBs/SNe are powered by magnetars. MNRAS443:67-71

McKee CF and Ostriker EC (2007) Theory of star formation. Annual Review of Astronomy and Astrophysics 45(1):565-687, https://doi.org/10.1146/annurev.astro.45.051806.110602

McKee CF and Ostriker JP (1977) A theory of the interstellar medium Three components regulated by supernova explosions in an inhomogeneous substrate. ApJ218:148-169

McKee CF and Zweibel EG (1992) On the Virial Theorem for Turbulent Molecular Clouds. ApJ399:551

Micic M, Glover SCO, Federrath C, et al. (2012) Modelling $\mathrm{H}_{2}$ formation in the turbulent interstellar medium: solenoidal versus compressive turbulent forcing. MNRAS421(3):2531-2542

Mierkiewicz EJ, Reynolds RJ, Roesler FL, et al. (2006) Detection of Diffuse Interstellar [O II] Emission from the Milky Way Using Spatial Heterodyne Spectroscopy. ApJ650(1):L63-L66

Mihalas D and Mihalas BW (1984) Foundations of radiation hydrodynamics

Minkowski R (1941) Spectra of Supernovae. PASP53(314):224

Mittal A and Girimaji SS (2019) Mathematical framework for analysis of internal energy dynamics and spectral distribution in compressible turbulent flows. Phys Rev Fluids 4:042601, https://link.aps.org/doi/10.1103/PhysRevFluids.4.042601

Miura H and Kida S (1995) Acoustic energy exchange in compressible turbulence. Physics of Fluids 7(7):1732-1742

Miville-Deschênes MA, Murray N, and Lee EJ (2017) Physical Properties of Molecular Clouds for the Entire Milky Way Disk. ApJ834:57 
Molina FZ, Glover SCO, Federrath C, et al. (2012) The density variance-Mach number relation in supersonic turbulence - I. Isothermal, magnetized gas. MNRAS423:2680-2689

Momferratos G, Lesaffre P, Falgarone E, et al. (2014) Turbulent energy dissipation and intermittency in ambipolar diffusion magnetohydrodynamics. MNRAS443(1):86-101

Morris M and Serabyn E (1996) The Galactic Center Environment. ARA\&A34:645-702

Mouschovias TC and Paleologou EV (1981) Ambipolar diffusion in interstellar clouds - Time-dependent solutions in one spatial dimension. ApJ246:48-64

Mouschovias TC and Spitzer L Jr (1976) Note on the collapse of magnetic interstellar clouds. ApJ210:326--

Moyal JE (1952) The spectra of turbulence in a compressible fluid; eddy turbulence and random noise. Mathematical Proceedings of the Cambridge Philosophical Society 48(2):329344

Müller B, Heger A, Liptai D, et al. (2016) A simple approach to the supernova progenitor-explosion connection. MNRAS460(1):742-764

Naab T and Ostriker JP (2017) Theoretical Challenges in Galaxy Formation. ARA\&A55:59-109

Nagai T, Inutsuka Si, and Miyama SM (1998) An Origin of Filamentary Structure in Molecular Clouds. ApJ506(1):306-322

Nakamura F and Li ZY (2007) Protostellar Turbulence Driven by Collimated Outflows. The Astrophysical Journal 662(1):395-412

Nakano T and Nakamura T (1978) Gravitational Instability of Magnetized Gaseous Disks 6. PASJ30:671-680

Obukhov AM (1941) On the distribution of energy in the spectrum of turbulent flow. Akademiia Nauk SSSR Doklady 32:22-24

Offner SSR and Arce HG (2014) Investigations of Protostellar Outflow Launching and Gas Entrainment: Hydrodynamic Simulations and Molecular Emission. The Astrophysical Journal 784(1):61

Offner SSR and Chaban J (2017) Impact of Protostellar Outflows on Turbulence and Star Formation Efficiency in Magnetized Dense Cores. The Astrophysical Journal 847(2):104

Offner SSR and McKee CF (2011) The Protostellar Luminosity Function. ApJ736(1):53

Offner SSR, Clark PC, Hennebelle P, et al. (2014) The Origin and Universality of the Stellar Initial Mass Function. Protostars and Planets VI pp 53-75

Onsager L (1949) Statistical hydrodynamics. Il Nuovo Cimento 6(2):279-287

Oppenheimer BD and Schaye J (2013) Non-equilibrium ionization and cooling of metal-enriched gas in the presence of a photoionization background. MNRAS434(2):1043-1062

Orkisz JH, Pety J, Gerin M, et al. (2017) Turbulence and star formation efficiency in molecular clouds: solenoidal versus compressive motions in Orion B. A\&A599:A99

Orr ME, Hayward CC, Hopkins PF, et al. (2018) What FIREs up star formation: the emergence of the Kennicutt-Schmidt law from feedback. 
MNRAS478(3):3653-3673

Osterbrock DE (1989) Astrophysics of gaseous nebulae and active galactic nuclei

Ostriker EC and Shetty R (2011) Maximally Star-forming Galactic Disks. I. Starburst Regulation Via Feedback-driven Turbulence. ApJ731:41

Ostriker EC and Shu FH (1995) Magnetocentrifugally Driven Flows from Young Stars and Disks. IV. The Accretion Funnel and Dead Zone. ApJ 447:813

Ostriker EC, McKee CF, and Leroy AK (2010) Regulation of Star Formation Rates in Multiphase Galactic Disks: A Thermal/Dynamical Equilibrium Model. ApJ721:975-994

Ostriker J (1964) The Equilibrium of Polytropic and Isothermal Cylinders. ApJ140:1056

Padoan P, Zweibel E, and Nordlund $\AA$ (2000) Ambipolar Drift Heating in Turbulent Molecular Clouds. ApJ540(1):332-341

Padoan P, Jimenez R, Nordlund A, et al. (2004) Structure Function Scaling in Compressible Super-Alfvénic MHD Turbulence. PhysRevLett92(19):191102

Padoan P, Federrath C, Chabrier G, et al. (2014) The Star Formation Rate of Molecular Clouds. In: Beuther H, Klessen RS, Dullemond CP, et al. (eds) Protostars and Planets VI, p 77

Padovani M and Galli D (2011) Effects of magnetic fields on the cosmic-ray ionization of molecular cloud cores. A\&A530:A109

Padovani M, Galli D, and Glassgold AE (2009) Cosmic-ray ionization of molecular clouds. A\&A501:619-631

Padovani M, Hennebelle P, and Galli D (2013) Cosmic-ray ionisation in collapsing clouds. A\&A560:A114

Padovani M, Ivlev AV, Galli D, et al. (2018) Cosmic-ray ionisation in circumstellar discs. A\&A614:A111

Palau A, Zapata LA, Román-Zúñiga CG, et al. (2018) Thermal Jeans Fragmentation within 1000 au in OMC-1S. ApJ855(1):24

Pan L and Padoan P (2009) The Temperature of Interstellar Clouds from Turbulent Heating. ApJ692(1):594-607

Pan L, Padoan P, Haugbølle T, et al. (2016) Supernova Driving. II. Compressive Ratio in Molecular-cloud Turbulence. ApJ825(1):30

Pardi A, Girichidis P, Naab T, et al. (2017) The impact of magnetic fields on the chemical evolution of the supernova-driven ISM. MNRAS465:4611-4633

Parker EN (1958) Dynamics of the Interplanetary Gas and Magnetic Fields. ApJ128:664

Pelletier G and Pudritz RE (1992) Hydromagnetic Disk Winds in Young Stellar Objects and Active Galactic Nuclei. ApJ394:117

Penston MV (1969) Dynamics of self-gravitating gaseous spheres-III. Analytical results in the free-fall of isothermal cases. MNRAS144:425

Peters T, Klessen RS, Mac Low M, et al. (2010a) Limiting Accretion onto Massive Stars by Fragmentation-induced Starvation. ApJ725:134-145

Peters T, Mac Low M, Banerjee R, et al. (2010b) Understanding Spatial and Spectral Morphologies of Ultracompact H II Regions. ApJ719:831-843 
Peters T, Klaassen PD, Mac Low MM, et al. (2014) Collective Outflow from a Small Multiple Stellar System. ApJ788:14

Pineda JL, Langer WD, Velusamy T, et al. (2013) A Herschel [C ii] Galactic plane survey. I. The global distribution of ISM gas components. A\&A554:A103

Piontek RA and Ostriker EC (2004) Thermal and Magnetorotational Instability in the Interstellar Medium: Two-dimensional Numerical Simulations. ApJ601:905-920

Planck Collaboration, Aghanim N, Akrami Y, et al. (2018) Planck 2018 results. XII. Galactic astrophysics using polarized dust emission. arXiv eprints arXiv:1807.06212

Porter D, Pouquet A, and Woodward P (2002) Measures of intermittency in driven supersonic flows. Phys. Rev. E66(2):026301

Porter DH, Woodward PR, and Pouquet A (1998) Inertial range structures in decaying compressible turbulent flows. Physics of Fluids 10(1):237-245

Prasad SS and Tarafdar SP (1983) UV radiation field inside dense clouds - Its possible existence and chemical implications. ApJ267:603-609

Price DJ, Tricco TS, and Bate MR (2012) Collimated jets from the first core. MNRAS423(1):L45-L49

Rees MJ (1976) Opacity-limited hierarchical fragmentation and the masses of protostars. MNRAS176:483-486

Reipurth B and Bally J (2001) Herbig-Haro Flows: Probes of Early Stellar Evolution. Annual Review of Astronomy and Astrophysics 39:403-455

Reynolds RJ (1989) The Column Density and Scale Height of Free Electrons in the Galactic Disk. ApJ339:L29

Reynolds RJ, Scherb F, and Roesler FL (1973) Observations of Diffuse Galactic HA and [n II] Emission. ApJ185:869-876

Richardson L (1965) Weather Prediction by Numerical Process. Dover books explaining science, Dover Publications, https://books.google.com/books?id=I4u_AAAAIAAJ

Richings AJ, Schaye J, and Oppenheimer BD (2014) Non-equilibrium chemistry and cooling in the diffuse interstellar medium - I. Optically thin regime. MNRAS440(4):3349-3369

Robitaille TP (2011) HYPERION: an open-source parallelized threedimensional dust continuum radiative transfer code. A\&A536:A79

Rodriguez LF, Moran JM, Ho PTP, et al. (1980) Radio observations of water vapor, hydroxyl, silicon monoxide, ammonia, carbon monoxide, and compact H II regions in the vicinities of suspected Herbig-Haro objects. The Astrophysical Journal 235:845-865

Rogers H and Pittard JM (2013) Feedback from winds and supernovae in massive stellar clusters - I. Hydrodynamics. MNRAS431(2):1337-1351

Roser JE, Swords S, Vidali G, et al. (2003) Measurement of the Kinetic Energy of Hydrogen Molecules Desorbing from Amorphous Water Ice. ApJ596(1):L55-L58

Rybicki GB and Lightman AP (1986) Radiative Processes in Astrophysics 
Sagaut P and Cambon C (2018) Homogeneous Turbulence Dynamics, 2nd edn. Springer International Publishing AG, Gewerbestrasse 11, 6330 Cham, Switzerland

Salpeter EE (1955) The Luminosity Function and Stellar Evolution. ApJ121:161

Sarkar S, Erlebacher G, Hussaini MY, et al. (1991) The analysis and modelling of dilatational terms in compressible turbulence. Journal of Fluid Mechanics $227: 473-493$

Schaye J (2004) Star Formation Thresholds and Galaxy Edges: Why and Where. ApJ609:667-682

Schinnerer E, Hughes A, Leroy A, et al. (2019) The Gas-Star Formation Cycle in Nearby Star-forming Galaxies. I. Assessment of Multi-scale Variations. ApJ887(1):49

Schmidt M (1959) The Rate of Star Formation. ApJ129:243

Schmidt W and Grete P (2019) Kinetic and internal energy transfer in implicit large-eddy simulations of forced compressible turbulence. Phys. Rev. E100(4):043116

Schmidt W, Federrath C, and Klessen R (2008) Is the scaling of supersonic turbulence universal? Phys Rev Lett 101:194505, https://link.aps.org/doi/10.1103/PhysRevLett.101.194505

Schneider N, Bontemps S, Girichidis P, et al. (2015) Detection of two power-law tails in the probability distribution functions of massive GMCs. MNRAS453:L41-L45

Schöier FL, van der Tak FFS, van Dishoeck EF, et al. (2005) An atomic and molecular database for analysis of submillimetre line observations. A\&A432(1):369-379

Schruba A, Leroy AK, Walter F, et al. (2010) The Scale Dependence of the Molecular Gas Depletion Time in M33. ApJ722:1699-1706

Schwarz C, Beetz C, Dreher J, et al. (2010) Lyapunov exponents and information dimension of the mass distribution in turbulent compressible flows. Physics Letters A 374(8):1039-1042

Seifried D, Schmidt W, and Niemeyer JC (2011) Forced turbulence in thermally bistable gas: a parameter study. A\&A526:A14

Seifried D, Pudritz RE, Banerjee R, et al. (2012) Magnetic fields during the early stages of massive star formation - II. A generalized outflow criterion. MNRAS422(1):347-366

Shaviv NJ and Regev O (1994) Interface dynamics and domain growth in thermally bistable fluids. Phys. Rev. E50(3):2048-2056

Shepherd DS and Churchwell E (1996) Bipolar Molecular Outflows in Massive Star Formation Regions. The Astrophysical Journal 472:225

Shu FH (1977) Self-similar collapse of isothermal spheres and star formation. ApJ214:488-497

Shu FH (1992) The physics of astrophysics. Volume II: Gas dynamics.

Shu FH, Lizano S, Ruden SP, et al. (1988) Mass Loss from Rapidly Rotating Magnetic Protostars. ApJ328:L19 
Shu FH, Najita J, Ostriker EC, et al. (1995) Magnetocentrifugally Driven Flows from Young Stars and Disks. V. Asymptotic Collimation into Jets. The Astrophysical Journal 455:L155

Skilling J and Strong AW (1976) Cosmic ray exclusion from dense molecular clouds. A\&A53(2):253-258

Slyz AD, Devriendt JEG, Bryan G, et al. (2005) Towards simulating star formation in the interstellar medium. MNRAS356:737-752

Smartt SJ (2009) Progenitors of Core-Collapse Supernovae. ARA\&A47(1):63106

Smith N (2014) Mass Loss: Its Effect on the Evolution and Fate of High-Mass Stars. ARA\&A52:487-528

Snell RL, Loren RB, and Plambeck RL (1980) Observations of CO in L 1551 : evidence for stellar wind driven shocks. The Astrophysical Journal 239:L17L22

Somerville RS and Davé R (2015) Physical Models of Galaxy Formation in a Cosmological Framework. ARA\&A53:51-113

Spitzer L Jr (1942) The Dynamics of the Interstellar Medium. III. Galactic Distribution. ApJ95:329

Spruit HC (2013) Essential Magnetohydrodynamics for Astrophysics. arXiv e-prints arXiv: 1301.5572

Stacy A and Bromm V (2013) Constraining the statistics of Population III binaries. MNRAS433(2):1094-1107

Stacy A, Bromm V, and Lee AT (2016) Building up the Population III initial mass function from cosmological initial conditions. MNRAS462(2):13071328

Stahler SW, Shu FH, and Taam RE (1980) The evolution of protostars. I Global formulation and results. ApJ241:637-654

Stecher TP and Williams DA (1967) Photodestruction of Hydrogen Molecules in H I Regions. ApJ149:L29

Stephens TL and Dalgarno A (1973) Kinetic Energy in the Spontaneous Radiative Dissociation of Molecular Hydrogen. ApJ186:165-168

Sternberg A and Dalgarno A (1995) Chemistry in Dense Photon-dominated Regions. ApJS99:565

Sternberg A, Le Petit F, Roueff E, et al. (2014) H I-to-H ${ }_{2}$ Transitions and H I Column Densities in Galaxy Star-forming Regions. ApJ790:10

Stone JM and Zweibel EG (2010) Ambipolar Diffusion-mediated Thermal Fronts in the Neutral Interstellar Medium. ApJ724(1):131-139

Stone JM, Ostriker EC, and Gammie CF (1998) Dissipation in Compressible Magnetohydrodynamic Turbulence. ApJ508(1):L99-L102

Strong AW, Moskalenko IV, and Ptuskin VS (2007) Cosmic-Ray Propagation and Interactions in the Galaxy. Annual Review of Nuclear and Particle Science 57:285-327

Subramanian K (2019) From Primordial Seed Magnetic Fields to the Galactic Dynamo. Galaxies 7(2):47

Sun J, Leroy AK, Schruba A, et al. (2018) Cloud-scale Molecular Gas Properties in 15 Nearby Galaxies. ApJ860:172 
Susa H (2013) The Mass of the First Stars. ApJ773(2):185

Sytine IV, Porter DH, Woodward PR, et al. (2000) Convergence Tests for the Piecewise Parabolic Method and Navier-Stokes Solutions for Homogeneous Compressible Turbulence. Journal of Computational Physics 158(2):225-238

Takahashi S, Ho PTP, Teixeira PS, et al. (2013) Hierarchical Fragmentation of the Orion Molecular Filaments. ApJ763(1):57

Tanaka KEI, Tan JC, and Zhang Y (2017) The Impact of Feedback During Massive Star Formation by Core Accretion. ApJ835(1):32

Tegmark M, Silk J, Rees MJ, et al. (1997) How Small Were the First Cosmological Objects? ApJ474:1

Tielens AGGM (2010) The Physics and Chemistry of the Interstellar Medium

Tielens AGGM and Hollenbach D (1985) Photodissociation regions. I. Basic model. ApJ291:722-746

Tomida K, Tomisaka K, Matsumoto T, et al. (2010) Radiation Magnetohydrodynamics Simulation of Proto-stellar Collapse: Two-component Molecular Outflow. ApJ714(1):L58-L63

Toomre A (1964) On the gravitational stability of a disk of stars. ApJ139:12171238

Troland TH and Crutcher RM (2008) Magnetic Fields in Dark Cloud Cores: Arecibo OH Zeeman Observations. ApJ680(1):457-465

Troland TH and Heiles C (1986) Interstellar magnetic field strengths and gas densities Observational and theoretical perspectives. ApJ301:339-345

van der Kruit PC and Freeman KC (2011) Galaxy Disks. ARA\&A49(1):301371

van der Tak FFS and van Dishoeck EF (2000) Limits on the cosmic-ray ionization rate toward massive young stars. A\&A358:L79-L82

van Dishoeck EF (1987) Photodissociation processes of astrophysical molecules. In: Vardya MS and Tarafdar SP (eds) Astrochemistry, IAU Symposium, vol 120, pp 51-65

van Dishoeck EF and Black JH (1988) The Photodissociation and Chemistry of Interstellar CO. ApJ334:771

van Loo S, Falle SAEG, Hartquist TW, et al. (2007) Shock-triggered formation of magnetically-dominated clouds. A\&A471:213-218

Veilleux S, Cecil G, and Bland-Hawthorn J (2005) Galactic Winds. ARA\&A43:769-826

Vink JS, de Koter A, and Lamers HJGLM (2001) Mass-loss predictions for O and B stars as a function of metallicity. A\&A369:574-588

Visser R, van Dishoeck EF, and Black JH (2009) The photodissociation and chemistry of $\mathrm{CO}$ isotopologues: applications to interstellar clouds and circumstellar disks. A\&A503(2):323-343

von Weizsäcker CF (1951) The Evolution of Galaxies and Stars. ApJ114:165

Wagner R, Falkovich G, Kritsuk AG, et al. (2012) Flux correlations in supersonic isothermal turbulence. Journal of Fluid Mechanics 713:482-490

Walch S, Girichidis P, Naab T, et al. (2015) The SILCC (SImulating the LifeCycle of molecular Clouds) project - I. Chemical evolution of the supernovadriven ISM. MNRAS454:238-268 
Walch SK, Whitworth AP, Bisbas T, et al. (2012) Dispersal of molecular clouds by ionizing radiation. MNRAS427:625-636

Wang J, Wang LP, Xiao Z, et al. (2010a) A hybrid numerical simulation of isotropic compressible turbulence. Journal of Computational Physics 229(13):5257-5279

Wang J, Shi Y, Wang LP, et al. (2012) Scaling and Statistics in ThreeDimensional Compressible Turbulence. PhysRevLett108(21):214505

Wang J, Yang Y, Shi Y, et al. (2013) Cascade of kinetic energy in three-dimensional compressible turbulence. Phys Rev Lett 110:214505, https://link.aps.org/doi/10.1103/PhysRevLett.110.214505

Wang J, Gotoh T, and Watanabe T (2017a) Scaling and intermittency in compressible isotropic turbulence. Physical Review Fluids 2(5):053401

Wang J, Gotoh T, and Watanabe T (2017b) Shocklet statistics in compressible isotropic turbulence. Physical Review Fluids 2(2):023401

Wang J, Gotoh T, and Watanabe T (2017c) Spectra and statistics in compressible isotropic turbulence. Physical Review Fluids 2(1):013403

Wang J, Wan M, Chen S, et al. (2019) Cascades of temperature and entropy fluctuations in compressible turbulence. Journal of Fluid Mechanics $867: 195-215$

Wang P, Li ZY, Abel T, et al. (2010b) Outflow Feedback Regulated Massive Star Formation in Parsec-Scale Cluster-Forming Clumps. The Astrophysical Journal 709(1):27-41

Ward JL, Kruijssen JMD, and Rix HW (2019) Not all stars form in clusters - Gaia-DR2 uncovers the origin of OB associations. MNRAS submitted arXiv:1910.06974

Webb GM, Axford WI, and Forman MA (1985) Cosmic-ray acceleration at stellar wind terminal shocks. ApJ298:684-709

Weber EJ and Davis J Leverett (1967) The Angular Momentum of the Solar Wind. ApJ148:217-227

Weingartner JC and Draine BT (2001a) Dust Grain-Size Distributions and Extinction in the Milky Way, Large Magellanic Cloud, and Small Magellanic Cloud. ApJ548(1):296-309

Weingartner JC and Draine BT (2001b) Photoelectric Emission from Interstellar Dust: Grain Charging and Gas Heating. ApJS134(2):263-281

Whelan ET, Ray TP, Podio L, et al. (2009) Classical T Tauri-like Outflow Activity in the Brown Dwarf Mass Regime. The Astrophysical Journal 706(2):1054-1068

White TG, Oliver MT, Mabey P, et al. (2019) Supersonic plasma turbulence in the laboratory. Nature Communications 10:1758

Whitworth A and Summers D (1985) Self-similar condensation of spherically symmetric self-gravitating isothermal gas clouds. MNRAS214:1-25

Whitworth A, Bate MR, Nordlund $\AA$, et al. (2007) The Formation of Brown Dwarfs: Theory. In: Reipurth B, Jewitt D, and Keil K (eds) Protostars and Planets V, p 459

Whitworth AP and Stamatellos D (2006) The minimum mass for star formation, and the origin of binary brown dwarfs. A\&A458(3):817-829 
Wiener J, Zweibel EG, and Oh SP (2013) Cosmic Ray Heating of the Warm Ionized Medium. ApJ767:87

Wiener N (1930) Generalized harmonic analysis. Acta Math 55:117-258, https://doi.org/10.1007/BF02546511

Wiersma RPC, Schaye J, and Smith BD (2009) The effect of photoionization on the cooling rates of enriched, astrophysical plasmas. MNRAS393(1):99107

Wolfire MG, Hollenbach D, McKee CF, et al. (1995) The Neutral Atomic Phases of the Interstellar Medium. ApJ443:152

Wolfire MG, McKee CF, Hollenbach D, et al. (2003) Neutral Atomic Phases of the Interstellar Medium in the Galaxy. ApJ587:278-311

Wurster J and Li ZY (2018) The role of magnetic fields in the formation of protostellar discs. Frontiers in Astronomy and Space Sciences 5:39

Yaglom AM (1949) The field of acceleration in turbulent flow. Dokl Akad Nauk SSSR 67(5):795-798

Yeung PK (2019) Advancing understanding of turbulence through extremescale computation. In: APS Division of Fluid Dynamics Meeting Abstracts, APS Meeting Abstracts, p E01.001

Yeung PK, Zhai XM, and Sreenivasan KR (2015) Extreme events in computational turbulence. Proceedings of the National Academy of Sciences of the United States of America 112(41):12633-12638, https://www.jstor.org/stable/26465472

Yorke HW and Sonnhalter C (2002) On the Formation of Massive Stars. ApJ569(2):846-862

Zhang Q, Hunter TR, Brand J, et al. (2005) Search for CO Outflows toward a Sample of 69 High-Mass Protostellar Candidates. II. Outflow Properties. The Astrophysical Journal 625(2):864-882

Zhang Y, Arce HG, Mardones D, et al. (2016) ALMA Cycle 1 Observations of the HH46/47 Molecular Outflow: Structure, Entrainment, and Core Impact. The Astrophysical Journal 832(2):158

Zhang Y, Arce HG, Mardones D, et al. (2019) An Episodic Wide-angle Outflow in HH 46/47. arXiv e-prints arXiv:1908.00689

Zhao D and Aluie H (2018) Inviscid criterion for decomposing scales. Physical Review Fluids 3(5):054603

Zrake J and MacFadyen AI (2012) Numerical Simulations of Driven Relativistic Magnetohydrodynamic Turbulence. ApJ744(1):32

Zweibel EG (2013) The microphysics and macrophysics of cosmic rays. Physics of Plasmas 20(5):055501

Zweibel EG (2017) The basis for cosmic ray feedback: Written on the wind. Physics of Plasmas 24(5):055402 